\title{
Profiling the inherent vulnerability of motor neuron subtypes
}

\author{
PhD thesis
}

in partial fulfilment of the requirements

for the degree

"Doctor rerum naturalium (Dr. rer. nat)"

Division of Mathematics and Natural Sciences

of the Georg August University Göttingen,

Faculty of Biology

Submitted by:

David Herholz

Born in

Datteln, Germany 
Supervisor, PhD committee member: Dr. Till Marquardt

Developmental Neurobiology

European Neuroscience Institute, Göttingen

PhD committee member: Prof. Dr. Nils Brose

Department of Molecular Neurobiology

Max Planck Institute for Experimental Medicine, Göttingen

PhD committee member: Prof. Dr. Michael Hörner

Department of Cell Biology

Johann-Friedrich-Blumenbach-Institute for Zoology and Anthropology, Göttingen

Date of submission of the PhD thesis: February 14, 2011 
I hereby declare that I prepared this $\mathrm{PhD}$ thesis, entitled "Profiling the inherent vulnerability of motor neuron subtypes", on my own and with no other sources and aids than those cited.

David Herholz

Göttingen, 14.2.2011. 


\section{Table of contents}

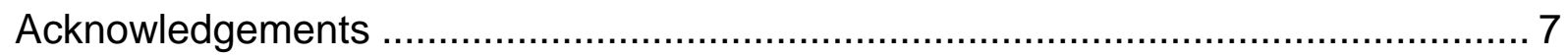

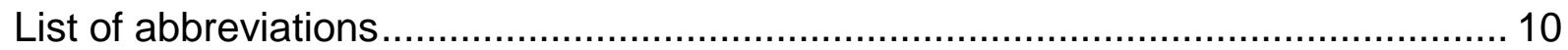

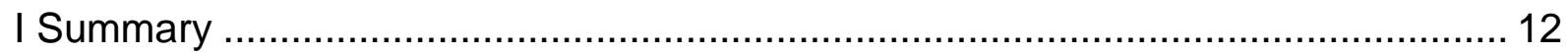

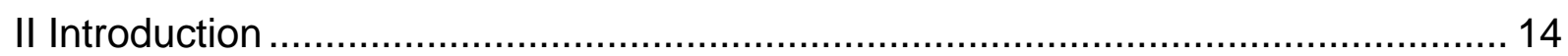

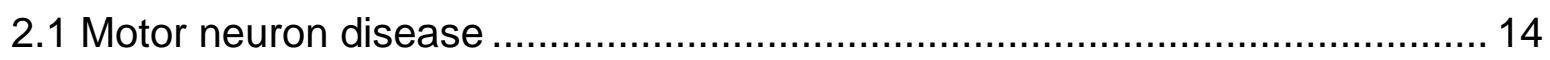

2.2 Amiotrophic lateral sclerosis ................................................................. 15

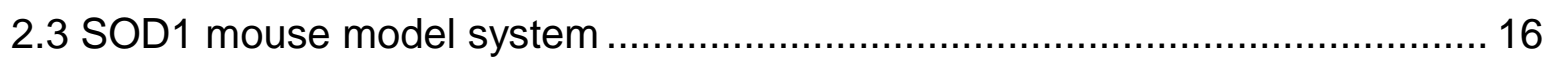

2.3.1 SOD1 mutation effects on mitochondria .............................................. 17

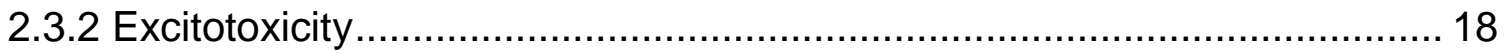

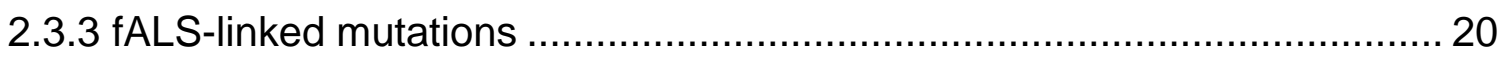

2.4 TDP-43: a more generalized marker for ALS …….................................... 21

2.5 RNA-binding as a link between neurological diseases.................................... 23

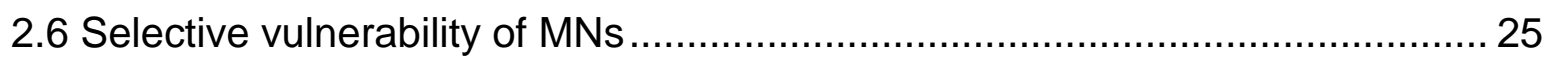

2.6.1 MN subtype specific vulnerability ....................................................... 26

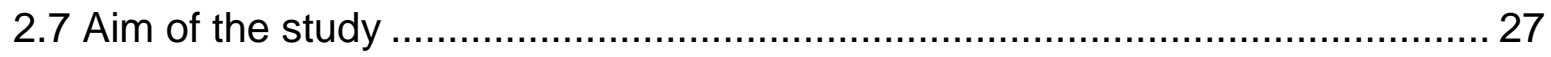

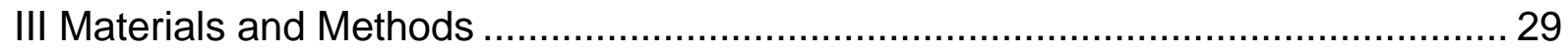

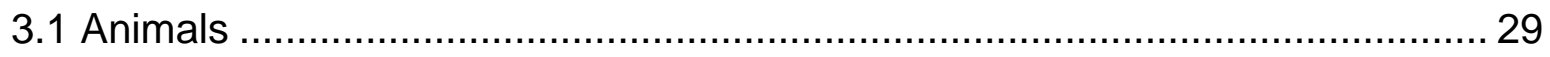

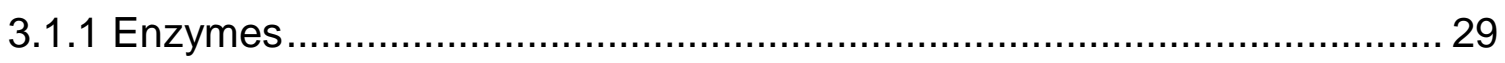

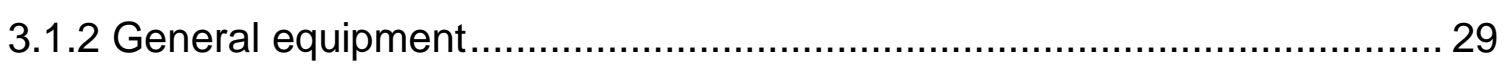

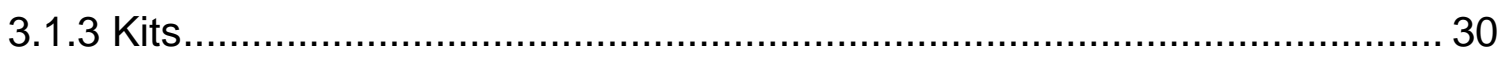

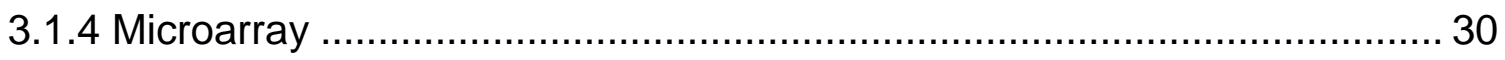

3.1.5 Chemicals/General reagents.......................................................... 30

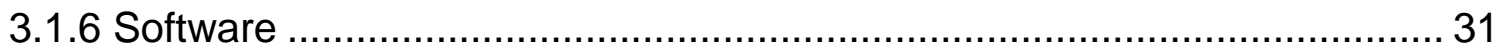

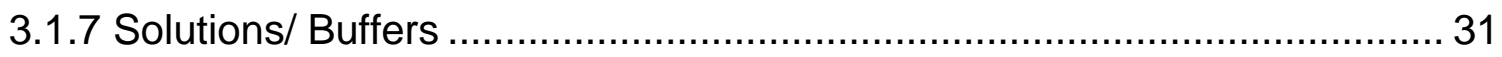

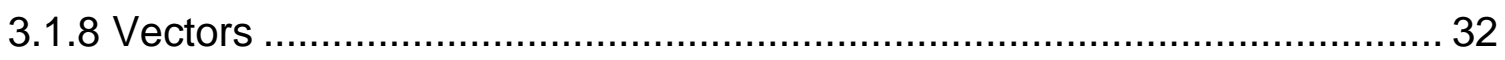

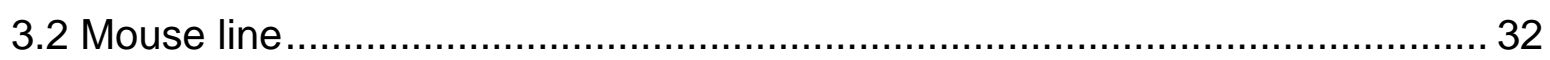

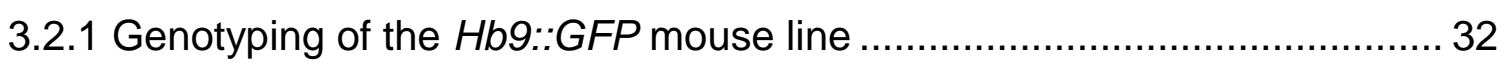

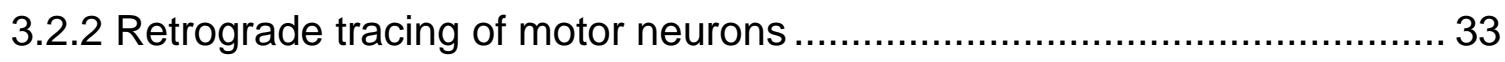

3.2.3 Tissue preparation for laser capture micro dissection ……...................... 33

3.2.4 Laser capture micro-dissection ...................................................... 34

3.2.5 RNA purification from captured cells....................................................... 35 


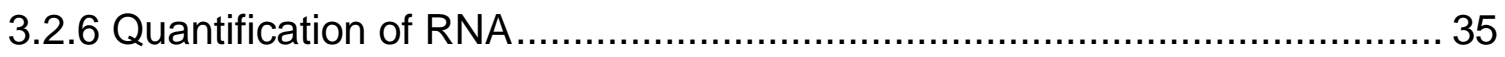

3.2.7 Amplification for microarray hybridization ............................................... 35

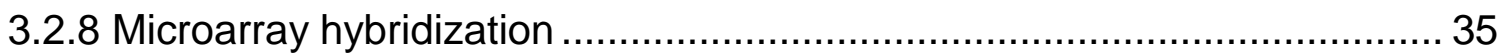

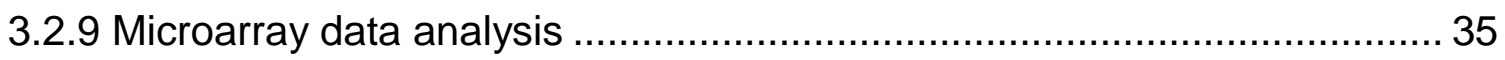

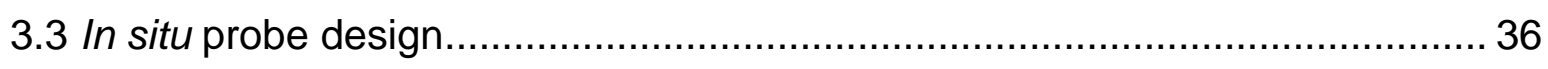

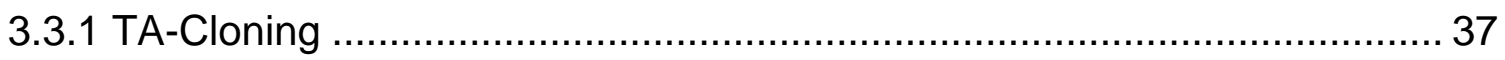

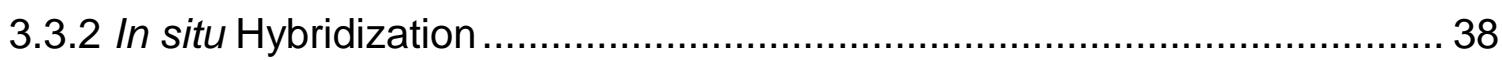

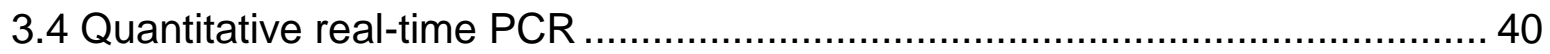

3.5 Long-term expression system in Chick embryo.......................................... 40

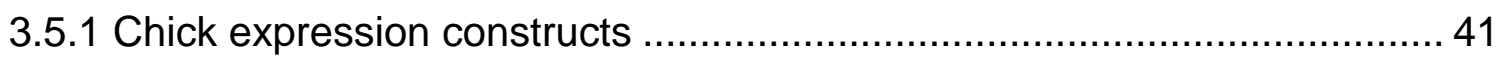

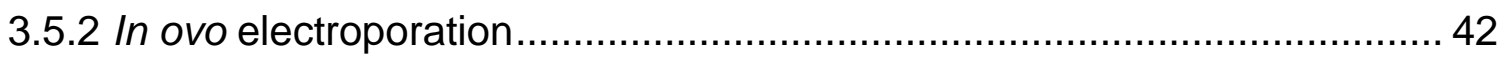

3.5.3 Chick embryo and tissue processing ................................................. 43

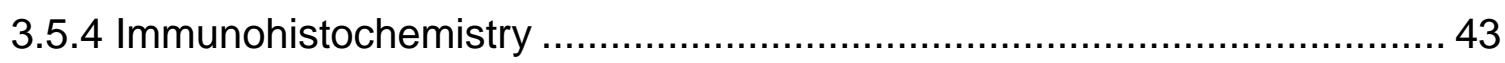

3.5.5 Microscopy and image analysis ........................................................ 44

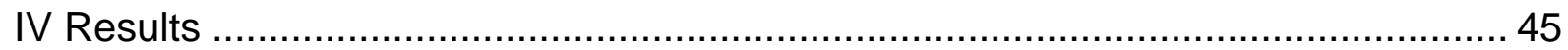

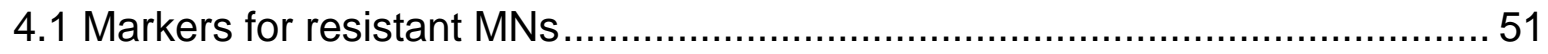

4.2 Functional network analysis of $\mathrm{S}$ candidates............................................. 55

4.3 Relevance of the array data for the vulnerability of MN subtypes ...................66

4.4 The Chick embryo as a model system to study ALS pathology in vivo............ 69

4.5 Verifying the role of candidate genes in protection from TDP-43 induced

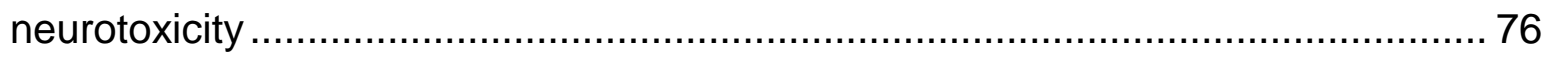

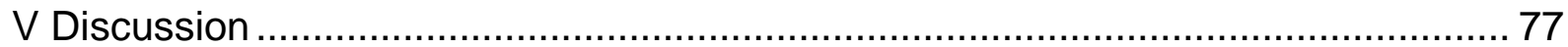

5.1 Genetic differences between MN subtypes ……...................................... 77

5.2 Relevance of differential gene expression to neuroprotection .......................... 80

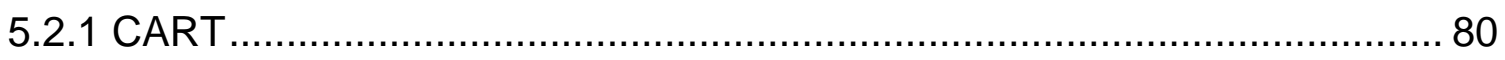

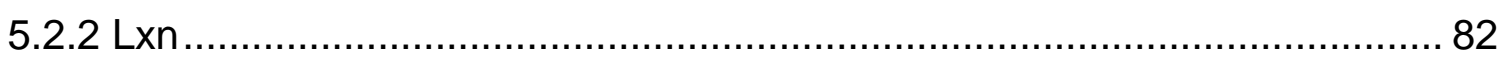

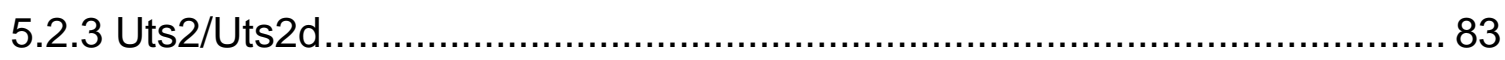

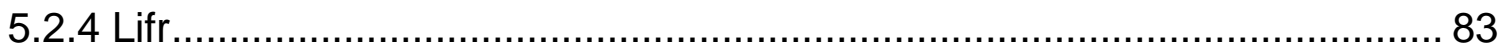

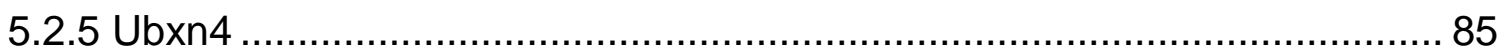

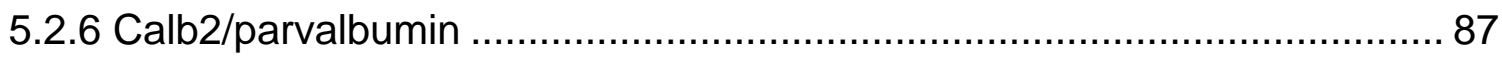

5.3 TDP-43 enhancer /suppressor model system .......................................... 88

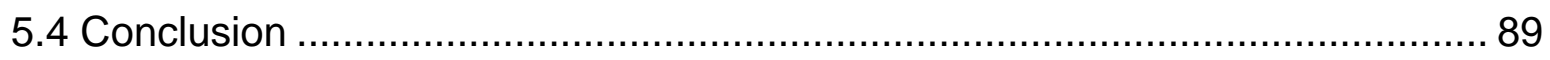

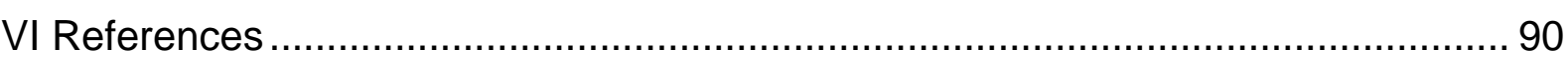

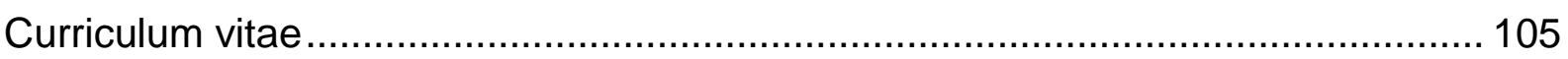


List of publications

106 


\section{Acknowledgements}

First of all I would like to thank my supervisor Dr. Till Marquardt. I could not think of a better support over the last three years than he provided me. It was sometimes hard to bear my stubbornness, but he always managed to stay calm and kept me on the ground. I do not only appreciate him as a supervisor but rather as a mentor. He made it easy to get me enthusiastic about my work. I hope that I could pay back at least a part of his trust that he put into me.

I would like to thank Prof. Dr. Nils Brose for his support as a member of my thesis committee. He always helped me out even though it was on short notice. I don't take this as granted. I loved playing soccer with him and moreover his really open minded attitude, being always straight forward and most of all honest.

I am grateful to Prof. Dr. Michael Hörner for his great way of calming me down every time I was in panic about organizational matters concerning my work and I am really grateful for his patience with me.

Dr. Aaron Voigt I want to thank especially for a master piece of work, that we did in collaboration. Voigt et al. 2010, that should make me unforgettable for him. I appreciated working with him and hope that we can go on and publish more in the future.

Further I would like to thank Prof. Dr. Jörg Schulz for his help with the publication of our paper.

I would like to thank Dr. Moritz Rossner for making a big part of my work possible.

Without his collaboration my thesis would have never produced such nice data. 
A special thanks goes to Dr. Sven Wichert. He introduced me to laser capture micro dissection and I would have never achieved my goals without his expertise. It was fun playing soccer with you and I hope we will meet again.

I would like to thank my programs the Göttingen Graduate School for Neurosciences and Molecular Biosciences (GGNB) and the Center for Molecular Physiology of the Brain (CMPB) and its coordinators for making a great job by giving students like me the opportunity of such a large variety of courses to improve knowledge.

This work would have never been possible without my great friend and colleague Daniel Müller. Erkenschwick!

Special gratitude goes to Alexandra Klusowski, it was great working with you and I really enjoyed having you around as the good spirit of the lab.

I thank Lukas Cyganek for having a great time, not only in the lab but also at sports. Moreover I would like to thank all people in the lab, Liang, Veltin, Ann, Pitchaiah, Eva and Heiko for bearing with me over the last years.

I would like to thank Team Erkenschwick: Beny, Chrissi and Claas. Erkenschwick rules! I hope that we will stay in touch forever.

Finally but not at the end I want to thank all my other friends, Mena, Eike, Christian, Bomba, Björn, Daniel, Robert, Anja, Kathi and many more.

Batti I would like to thank in particular for being my dearest friend. I wish you all the best in the world and beyond.

To my mother and my father, you cannot imagine how happy I am having the most supportive, patient and beloved parents in the world. I know that it was by far not easy with me and look what you made of me, thank you!

My sister and my brother I thank for their unconditional love. I am more than grateful to have you. 
Most importantly I would like to thank my girlfriend Marija. Thank you for all your love and support. You mean the world to me. 


\section{List of abbreviations}

\begin{tabular}{|c|c|}
\hline Aldoc & aldolase C \\
\hline ALS & amyotrophic lateral sclerosis \\
\hline Bex1 & brain expressed gene 1 \\
\hline$B C V$ & Bulbocavernosus \\
\hline Calb2 & calbindin 2 \\
\hline Cartpt & cocaine- and amphetamine-regulated transcript prepropeptide \\
\hline CREB & cAMP-response-element-binding protein \\
\hline CSF & cerebrospinal fluid \\
\hline CTxB & cholera toxin subunit B \\
\hline DCTN1 & the p150 subunit of dynactin \\
\hline Emb & Embigin \\
\hline ER & endoplasmic reticulum \\
\hline ERAD & ER-associated degradation \\
\hline ERK & extracellular signal regulated protein kinase \\
\hline fALS & familial amyotrophic lateral sclerosis \\
\hline FF & fast fatigable \\
\hline FTDP & frontotemporal dementia and Parkinson's disease \\
\hline FUS & Fused in sarcoma \\
\hline hnRNP & heterogeneous nuclear ribonucleoprotein \\
\hline HSP & hereditary spastic paraplegia \\
\hline IBMPFD & $\begin{array}{l}\text { Inclusion body myopathy associated with Paget's disease of bone and } \\
\text { frontotemporal dementia }\end{array}$ \\
\hline IMS & inner membrane space \\
\hline LIFR & leukemia inhibitory factor receptor \\
\hline Lxn & Latexin \\
\hline MAPT & microtubule associated protein \\
\hline MN & motor neuron \\
\hline MND & motor neuron disease \\
\hline MS & multiple sclerosis \\
\hline
\end{tabular}




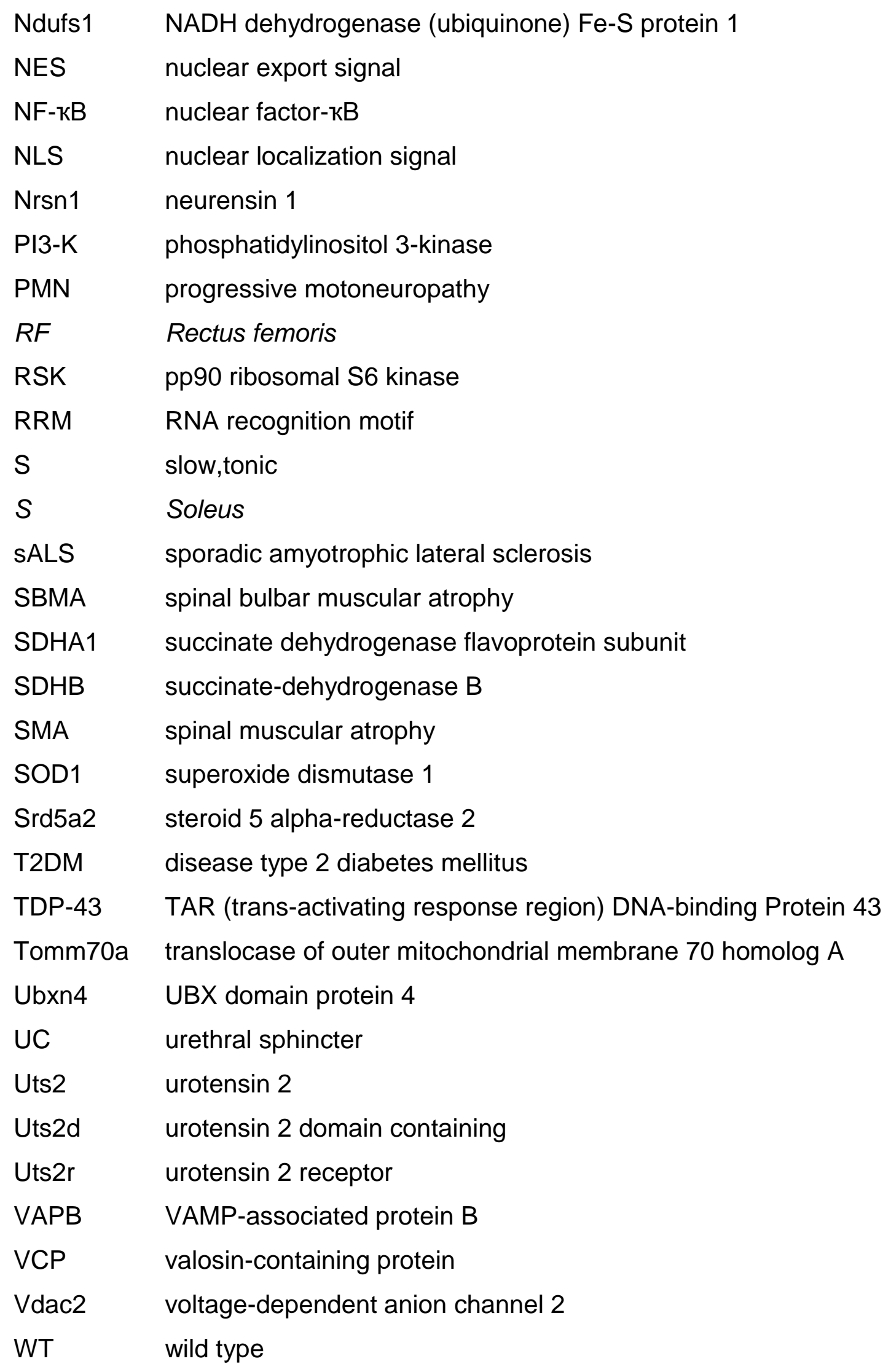




\section{Summary}

Motor neuron diseases (MNDs) are a heterogeneous group of disorders that result in selective degeneration of upper and/or lower motor neurons (MNs). The precise causes of most MNDs remain poorly resolved and treatment options are currently mostly limited to the easing of symptoms and intensive care efforts. Remarkably, distinct MN subtypes display dramatic differences regarding their vulnerability towards degeneration in MNDs. The mechanistic basis for these inherent differences of the MN subtypes remains unknown. In order to understand what renders different motor neuron subtypes vulnerable, or resistant the thesis project first aimed to identify genetic factors linked to the different MN subtype. To achieve this, a transcriptome-wide screen of vulnerable versus resistant $\mathrm{MN}$ subtypes was performed. Firstly, this comprised identifying the transcriptome of MNs innervating the $M$. Rectus femoris (RF), a predominantly fast muscle innervated by fast MNs that are highly susceptible to MNDs. Secondly, the $R F$ transcriptome was compared to that of the MNs innervating the $M$. Soleus $(S)$, a slow muscle innervated mainly by slow MNs that are relatively resistant towards MNDs. Thirdly, as an example for resistant MNs, the gene signatures of both the $R F$ and $S$ MNs were compared to the transcriptome of the MNs innervating the M. Bulbocavernosus (BCV) muscle. The results showed a highly differential expression profile between the resistant $B C V$ and $S$ MNs on the one hand and the vulnerable $(R F)$ MNs on the other. Promising candidate genes were defined by in silico data analysis and verified via QPCR and in situ hybridization. For instance, the identified genes Cartpt and Uts2/Uts2d provided the first confirmed molecular markers for resistant and relatively resistant MNs. Moreover, genes including Cart, Lxn, Lifr, Ubxn4, Calb2 and Pvalb that could be shown to be selectively expressed by resistant MNs have previously been linked to 
mediating neuroprotective roles. In parallel to the in silico analysis, all 200 identified candidate genes that showed an association with a resistant $\mathrm{MN}$ profile (high expressed in $B C V$ plus $S$, but low expression in $R F$ ) were systematically screened to identify potential modifiers of neurotoxicity in four separate neurodegeneration models in the fruitfly Drosophila melanogaster. This screen for instance identified 36 modifiers of TPD-43-mediated retinal neuron loss as a model for MND-linked neurodegeneration. Interestingly, a substantial portion of the pool of the 'MN resistance-associated' genes that showed a modifier activity in this screen, were linked to the ubiquiting/proteasome pathway. One of these genes encoded the ubiquitin ligase Ubxn4, a key co-factor of the central component of the ER-associated protein degradation pathway via VCP. Mutations in VCP in turn have recently been linked to familial MNDs and were shown to trigger aggregation of MND-linked TDP43. To allow confirming potential modifiers of MND-linked neurodengeneration a novel vertebrate model system for studying the MN loss in vivo was developed. This allowed stable neuron subtype-specific transgene expression of human TDP-43 in chick, which leads to progressive MN loss, in part mediated by caspase3-dependent apoptosis. This further showed for the first time that neurotoxicity mediated by the MND-linked protein TDP-43 requires its endogenous RNA binding activity. This provides the basis for systematically testing the activities of selected candidate genes on MND-like neurodegeneration of MNs in vivo. Taken together, this work revealed the first gene signatures and molecular markers associated with MNs that show resistance towards neurodegeneration in MNDs. In addition, the thesis project further provided the basis to test a defined set of these genes for a role in mediating MN resistance in MND models in vivo. 


\section{Introduction}

\subsection{Motor neuron disease}

Motor neuron diseases (MNDs) are a heterogeneous group of disorders that result in selective degeneration of upper and/or lower motor neurons (MNs). During the course of MND, MNs denervate and die progressively, leading to spasticity, muscle weakness, atrophy and ultimately fatality/death through respiratory failure. Spinal muscular atrophy (SMA) and spinal bulbar muscular atrophy (Kennedy's disease, SBMA) selectively affect lower MNs in the spinal cord, while hereditary spastic paraplegia (HSP) involves mostly upper MNs in the motor cortex (Gros-Louis et al., 2006). Amyotrophic lateral sclerosis (ALS) is the most common MND. It occurs in four to six people out of 100000 , and has an adult onset at typically $40-60$ years of age. ALS affects both upper and lower MNs and usually leads to death in about one to five years after disease onset (Mitsumoto, 1998; Pasinelli and Brown, 2006; Yoshida et al., 1986). Notably, there are no effective treatments available, with the only exception of Riluzole, which in some cases can slightly prolong survival (Bruijn et al., 2004). Treatment options for MNDs are currently restricted to intensive care efforts, such as physical therapy, specific diets, administration of muscle relaxants and tranquilizers. 


\subsection{Amiotrophic lateral sclerosis}

90-95\% of all ALS cases have no obvious family history and are known as sporadic ALS (sALS). Causes for sALS are still unknown. However, factors including environmental stress (toxic elements in soil, air, food, handled material, etc.), viral toxicity, injury and even professional football have been implicated to increase the risk of ALS (Chio et al., 2005). Approximately $5-10 \%$ of ALS cases are hereditary in an autosomal dominant manner and are termed familial ALS (fALS) (Rosen, 1993; Shaw et al., 2007). Importantly, the disease progression in fALS and SALS is indistinguishable, bearing the hallmark of denervation and progressive loss of MNs leading to death by respiration failure.

Even though a significant effort has been invested to discover the genes implicated in fALS, the genetic causes for the majority of ALS cases remain unclear. Until now, eight loci (ALS1-8) and six genes have been identified to be involved in ALS development. However, so far, no general mechanism has been found. All different ALS types seem to be triggered by different causes. For example, mutations in the superoxide dismutase 1 (SOD1) and the VAMP-associated protein B (VAPB), as well as the microtubule associated protein (MAPT), have been associated with ALS1, ALS8 and ALS-FTDP (ALS with frontotemporal dementia and Parkinson's disease), respectively. These proteins seem to play key roles in the toxic events occurring in these subtypes of ALS (Boillee et al., 2006).

It has been further shown that mutations in the gene encoding the SOD1 are involved in $\sim 20 \%$ of fALS and $3 \%$ of sALS cases (Rosen, 1993). More than 125 mutations in SOD1 that account for ALS have been found so far (Dion et al., 2009) and they can affect both the active site and the structure of the protein. The discovery of SOD1 mutations as one of the causes of ALS has led to development of different model 
systems for studying the molecular and genetic basis of ALS and has broadened our understanding of the development of the disease.

\subsection{SOD1 mouse model system}

SOD1 is a ubiquitously expressed and mostly soluble cytoplasmic protein, which converts the free radical species produced during mitochondrial respiration and other biological processes into molecular oxygen and hydrogen peroxide, thus protecting the cell from potentially harmful reactive oxygen species. SOD1 mutations discovered in ALS patients have been introduced in a mouse model system. The overexpression of mutated SOD1 mimics the disease progression of ALS in human patients by forming aggregates and leading to MN loss. Interestingly, SOD1 knockout mice show no MN degeneration. Furthermore, the toxic effect of SOD1 has been shown only in the cases of overexpression of mutated SOD1 forms, regardless whether the mutation affects its enzymatic activity (Bruijn et al., 1998; Deng et al., 2006; Jaarsma et al., 2000; Subramaniam et al., 2002). These findings point to a gain of function, rather than a loss of function model system in SOD1 mediated ALS toxicity.

Interestingly, it has been shown that mutated SOD1 expression restricted to MNs is not sufficient to trigger MN loss, similarly as the restricted expression to astrocytes, microglia or macrophage cells (Beers et al., 2006; Gong et al., 2000; Lino et al., 2002; Pramatarova et al., 2001). These findings implicate a non-cell-autonomous effect of SOD1 toxicity. Denervation of the muscles is one of the early events in disease progression. Moreover, SOD1 is expressed in muscle cells and it has been shown that exclusive expression of mutant SOD1 in muscles is sufficient to trigger MN loss in mice (Wong and Martin, 2010). Even though the mechanism underlying MN loss in SOD1 mouse model system has been intensively investigated, the precise biology of the MN degeneration is largely unclear. ALS-linked mutations of SOD1 
have been implicated in formation of intracellular aggregates of SOD1. Furthermore, these mutations have been linked to glutamate excitotoxicity, mitochondrial dysfunction, axonal transport defect and other disease mediated effects. It seems that defects in all these pathways converge to make a deleterious effect on MN survival.

\subsubsection{SOD1 mutation effects on mitochondria}

ALS-like pathology in the SOD1 model system seems to affect mitochondria in multiple ways and is therefore a major subject of investigation (Bruijn et al., 2004; Manfredi and $\mathrm{Xu}, 2005)$. Besides their primarily function in oxygen dependent ATP production, mitochondria are also involved in calcium buffering, calcium signalling and apoptosis. Mitochondrial function is regulated by mitochondrial biogenesis, transport, clearance, fusion and fission (Chu, 2010). Thereby, dysregulation of one mitochondrial pathway could severely affect the metabolism of MNs.

Besides the cytoplasm, wild type (WT) SOD1 has also been found in the nucleus (Crapo et al., 1992), the endoplasmatic reticulum (ER) (Kikuchi et al., 2006) and in the inner membrane space (IMS) of mitochondria (Okado-Matsumoto and Fridovich, 2001; Sturtz et al., 2001). Mislocalized mutated SOD1 has also been found in the IMS, mitochondrial matrix, outer membrane and the cytoplasmic surface of mitochondria (Higgins et al., 2002; Kawamata and Manfredi, 2008; Vande Velde et al., 2008; Vijayvergiya et al., 2005).

The mislocalization in various mitochondrial structures, of mutant SOD1 have been implicated in causing damage to the mitochondrial membrane, loss of membrane potential, swelling of mitochondria (Kong and Xu, 1998; Wong et al., 1995) and impairment of the respiratory complex (Borthwick et al., 1999; Bowling et al., 1993; Jung et al., 2002). This could decrease cellular ATP production (Ferri et al., 2006) 
and affect the motor neuron survival through energy homeostasis and loss of membrane potential. In support of these suggestions, the oral administration of creatine in SOD1 mutant mice, have been shown to delay the MN loss. Creatin works as a stabilizer of the mitochondrial creatine kinase, thereby constituting an intricate energy buffering and transport system. Taken altogether, these data suggest that the MN survival is at least in part dependent on mitochondrial metabolism (Klivenyi et al., 1999). Interestingly, mutant SOD1 protein has also been shown to be localized in the ER and form aggregates (Kikuchi et al., 2006). As a result it may disrupt the ERassociated degradation machinery and inducing ER-stress in MNs (Atkin et al., 2006; Nishitoh et al., 2008; Saxena et al., 2009).

\subsubsection{Excitotoxicity}

Excitotoxicity seems to play a major role in the vulnerability of MNs in ALS (Fig. 1). It has been shown that the glutamate levels in cerebrospinal fluid (CSF) are threefold higher than normal in ALS patients (Rothstein et al., 1990). High glutamate levels were shown to trigger MN death in vitro, whereas glutamate inhibitors and AMPA receptor antagonists protected against $\mathrm{MN}$ loss in vivo and in vitro in cultured organotypic spinal cord slices (Rothstein et al., 1996; Rothstein et al., 1993; Rothstein and Kuncl, 1995; Rothstein et al., 1992). Elevated levels of glutamate may result from a dysfunction of the glutamate transporter EAAT-2 located on astrocyte processes, which is responsible for clearing up the synaptic cleft upon glutamate release (Swanson et al., 1997). This is thought to lead to overstimulation of the ionotropic glutamate receptor and accumulation of high levels of $\mathrm{Ca}^{2+}$ in the MN. MNs are especially vulnerable towards high concentrations of $\mathrm{Ca}^{2+}$ due to the lack of calcium buffering proteins in MNs like parvalbumin or calbindin D-28k, which may cause excitotoxicity (De Maria et al., 1994; Ince et al., 1993). 


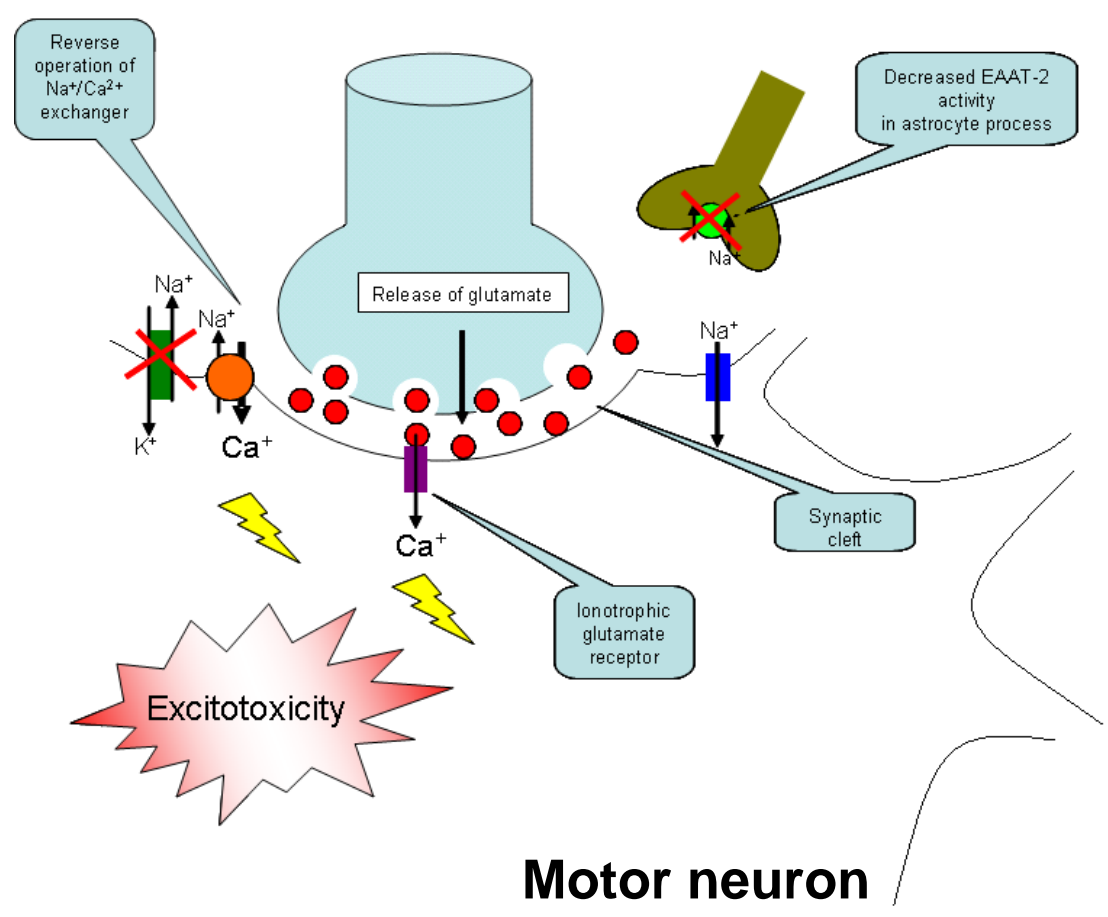

Fig. 1 Excitotoxicity in MNs

Pathological decreased activity of the glutamate transporter EAAT-2 in astrocyte processes increases the glutamate concentration in the synaptic cleft. Overstimulation of the ionotrophic glutamatergic receptors leads to high $\mathrm{Ca}^{2+}$ levels in the cytosol of the $\mathrm{MN}$, causing activation of degradation enzymes and impaired mitochondrial functions. Perturbations in mitochondria functions deplete ATP supply of the $\mathrm{Na}^{+} / \mathrm{K}^{+}$ATPase and therefore $\mathrm{Na}^{+}$transported out of the cell by exchange with $\mathrm{Ca}^{2+}$, exacerbating elevated $\mathrm{Ca}^{2+}$ levels and cause excitotoxicity (Cheah et al., 2010).

Increased levels of $\mathrm{Ca}^{2+}$ have been found in ALS patients, as well as in cell lines and SOD1 mouse models. These elevated levels have been proposed to lead to mitochondrial perturbations, since mitochondrial calcium buffering capability was found to be impaired in SOD1 ${ }^{\text {G93A }}$ mouse model MNs and neuroblastoma cell cultures (Jaiswal and Keller, 2009; Jaiswal et al., 2009). Subsequently, this could interrupt the supply of the $\mathrm{Na}^{+} / \mathrm{K}^{+}$ATPase with ATP, leading to accumulation of $\mathrm{Na}^{+}$ in the cytosol and cause hyperexcitability of the MN. In order to remove the accumulating $\mathrm{Na}^{+}$, more $\mathrm{Ca}^{2+}$ would be imported, (see Fig.1), and raise activation of degradative enzymes like proteases (Cheah et al., 2010). Furthermore, in SOD1 ${ }^{\text {G93A }}$ 
mice high levels of $\mathrm{Ca}^{2+}$ appear to induce apoptosis by release of mitochondrial cytrochrome c into the cytosol (Kirkinezos et al., 2005; Pasinelli et al., 2004; Takeuchi et al., 2002). Blocking of cytochrome c release with minocycline delays disease onset and prolongs survival of the mice (Zhu et al., 2002).

Consistent with the hypothesis of excitotoxicity being one of the mediators of MN death during ALS development, a defect in EAAT-2 expression has been found in $\sim 80 \%$ of the ALS patients (Rothstein and Kuncl, 1995). Furthermore, SOD1 mutant mice have a ca. $50 \%$ decrease in EAAT-2 expression. The overexpression of the protein has been shown to have a neuroprotective role and to delay MN death in SOD1 mutant mice (Guo et al., 2003). Moreover, free radicals can oxidize thiol groups and form disulfide bounds between cystein residues in the "redox sensing" domains of EAAT-2 in astrocytes (Trotti et al., 1998). These "redox sensing" domains appear to regulate the transport of glutamate through thiol-disulphide conversion. Therefore, oxidation of these "redox sensing" domains can lead to inhibition of EAAT2 (Volterra et al., 1994a; Volterra et al., 1994b; Volterra et al., 1994c), closing the cycle to elevated levels of glutamate in the CSF of ALS patients.

\subsection{3 fALS-linked mutations}

Besides mutations in SOD1, mutations in several other genes have been found in different forms of fALS. These genes include the G protein exchange factor ALS2 (Hadano et al., 2001; Yang et al., 2001), vesicle associated membrane protein B (VAPB) (Nishimura et al., 2004), sentaxin (Chen et al., 2007), the p150 subunit of dynactin (DCTN1) (Munch et al., 2004), angiogenin (Greenway et al., 2006), TDP-43 (Kabashi et al., 2008; Rutherford et al., 2008; Sreedharan et al., 2008), FUS and VCP (Gitcho et al., 2009; Kwiatkowski et al., 2009; Ritson et al., 2010; Vance et al., 2009). 


\subsection{TDP-43: a more generalized marker for ALS}

Recently, a new candidate has refocused attention towards a more generalized model system of ALS. The TAR (trans-activating response region) DNA-binding Protein 43 (TDP-43), was found to be linked to both sALS and fALS (Kabashi et al., 2008; Neumann et al., 2006; Sreedharan et al., 2008), thereby providing a potential key molecule to a more common understanding of ALS.

Aggregations of TDP-43 have been shown to occur in almost all cases of SALS and fALS (Neumann et al., 2006). Moreover, mutations in TDP-43 have also been found in a smaller subset of human ALS patients, six out of 120 sALS and 3 out of 80 fALS cases, respectively (Kabashi et al., 2008; Sreedharan et al., 2008). Thereby, defects in TDP-43 may represent a more generalized marker for ALS (Kwong et al., 2007).

TDP-43 is classified as a heterogeneous nuclear ribonucleoprotein (hnRNP) (Buratti and Baralle, 2001; Crozat et al., 1993; Wang et al., 2004) and is closely related to hnRNP-A1 which is a representative of a subclass of proteins containing two RNAbinding motifs followed by a glycine-rich domain (Dreyfuss et al., 1993; Matunis et al., 1992).

Accordingly, TDP-43 is a nuclear located protein with two RNA recognition motifs, a nuclear localization signal (NLS), as well as nuclear export signal (NES) (Fig.2). Nearly all mutations found associated with ALS are located in the conserved glycinerich domain of TDP-43 (Pesiridis et al., 2009). Glycine-rich domains of heterogeneous nuclear ribonucleoprotein family have been shown to promote protein-protein interactions, RNA binding and nucleocytoplasmatic shuttling (Cartegni et al., 1996; Mayeda et al., 1994; Siomi and Dreyfuss, 1995; Weighardt et al., 1995). Interestingly, most of the mutations involve amino acids that are highly conserved among mammals (Del Bo et al., 2009; Sreedharan et al., 2008; Yokoseki et al., 2008). 


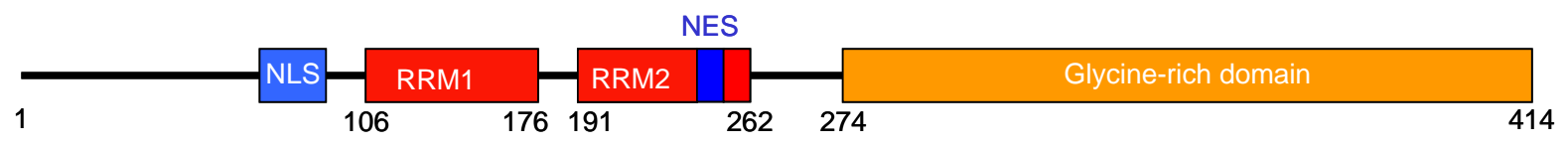

Fig. 2 Scheme of TAR DNA-binding Protein 43 (TDP-43)

TDP-43 is a $43 \mathrm{kDa}$ protein with nuclear localization. It harbours two RNA recognition motifs (RRM1,2), a nuclear localization signal (NLS), a nuclear export signal (NES) and a C-terminal glycine-rich domain, which carries nearly all mutations found in human patients of ALS.

Both, loss of function and gain of function models are controversially discussed to explain toxicity of TDP-43 aggregations and mutations. Moreover, expression models of multiple mutated or truncated forms of TDP-43 protein have been shown to cause motor deficits and toxicity in Zebrafish (Kabashi et al., 2008), Drosophila (Li et al., 2010; Miguel et al., 2011), chicken (Sreedharan et al., 2008; Voigt et al., 2010), rat (Tatom et al., 2009) and mouse (Wegorzewska et al., 2009) mimicking the disease progression in human patients. It has been suggested that in the chick embryo model system, Q331K and M337V mutations of TDP-43 have overall toxic effects on embryonic development, but that wild type (WT) TDP-43 transfection had no effect. These and other experiments suggested a toxic gain of function mechanism (Sreedharan et al., 2008). Q331K mutation also showed a higher affinity to aggregate in vitro (Johnson et al., 2009). However, multiple studies in several organisms like rat and mouse overexpressing human WT TDP-43 showed an apoptotic neuron loss (Igaz et al., 2011; Tatom et al., 2009). Similar observations could be made in TDP-43 knockout models in Drosophila and zebrafish (Feiguin et al., 2009; Kabashi et al., 2008), suggesting a loss of function mechanism. 


\subsection{RNA-binding as a link between neurological diseases}

Surprisingly, two other proteins that belong to the same superfamily as TDP-43 have been discovered to be involved in toxic events during ALS, or related diseases. Fused in sarcoma (FUS) and valosin-containing protein (VCP), both RNA-binding proteins, have been shown to carry mutations in ALS (FUS), or Inclusion body myopathy associated with Paget's disease of bone and frontotemporal dementia (IBMPFD) (VCP). The latter is a neurodegenerative disease associated with TDP-43 mislocalization (Gitcho et al., 2009; Kwiatkowski et al., 2009; Ritson et al., 2010; Vance et al., 2009) (Fig. 3 and 4). The functions of nuclear located FUS protein lie in the DNA/RNA metabolism where it is involved in transcription, RNA splicing and transport (Baechtold et al., 1999; Bertrand et al., 1999; Yang et al., 1998). Mutations in FUS have been found in the G-rich and RGG-rich domain of the protein and cause a mislocalization of FUS into the cytosol, where it forms aggregates which are linked to ALS pathology (Kwiatkowski et al., 2009; Vance et al., 2009). FUS is involved in about $5 \%$ of fALS cases that are negative for SOD1. So far, no FUS mutations have been found in sALS (Kwiatkowski et al., 2009).

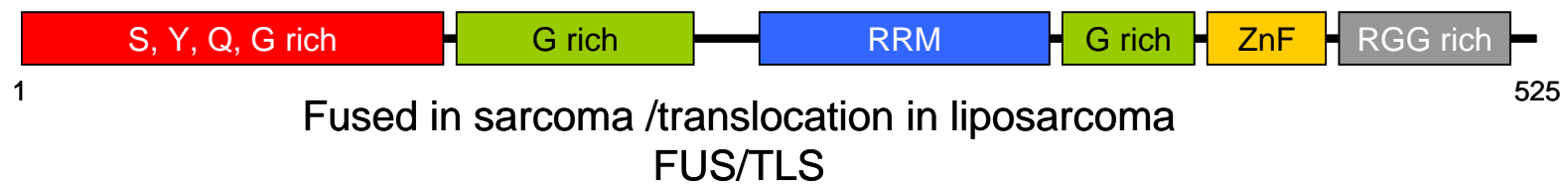

Fig. 3 Scheme of Fused in sarcoma/translocation in liposarcoma (FUS/TLS)

The RNA-binding protein FUS consists of a N-terminal S-Y-Q-G-rich domain, two G-rich domains, a RNA recognition motif (RRM), a zinc-finger domain and a $R G G$ rich C-terminal domain.

The second RNA-binding protein VCP is characterized as a highly conserved AAA ${ }^{+}-$ ATPase. VCP is involved in transport of the membrane fusion machinery to fusion 
events (Dreveny et al., 2004), enhancement of the ER-associated degradation (ERAD) (Ballar et al., 2006), DNA repair (Livingstone et al., 2005; Pye et al., 2007) and targeting of misfolded proteins to the proteasome (DeLaBarre et al., 2006; McCracken and Brodsky, 1996; Schmitz and Herzog, 2004). Therefore, VCP is implicated in the cell survival pathways and neuroprotection (Nowis et al., 2006; Vandermoere et al., 2006; Wojcik et al., 2006). Knock down of VCP, as well as mutations decrease cellular proteasome activity (Forman et al., 2006; Gitcho et al., 2008; Gitcho et al., 2009). Unlike FUS, VCP shows direct genetic interaction with TDP-43, whereby siRNA mediated knockdown of TDP-43 suppresses IBMPFD pathology caused by VCP mutation (Ritson et al., 2010), while mutations in TDP-43 enhance the pathology (Ritson et al., 2010). Disease related mutations in VCP can mediate a redistribution of TDP-43 into the cytosol, whereas mutated VCP mislocalizes into the nucleus (Gitcho et al., 2009; Ritson et al., 2010). In addition, VCP mutations have been found in fALS cases and are thought to be involved in 1$2 \%$ of all fALS cases. It has been postulated that mutations in VCP mediate toxicity through the impairment of the ubiquitination and protein degradation pathway in MNs (Johnson et al., 2009; Shaw et al., 2007).

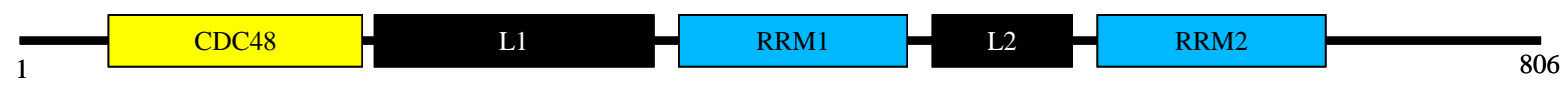

Valosin-containing protein (VCP)

Fig. 4 Scheme of Valosin-containing protein (VCP)

VCP consists of a N-terminal cofactor binding site (CDC48, cell division cycle protein 48), two linker domains and two RNA-recognition motifs (RRM1,-2)

So far, numerous mechanisms have been suspected to cause the toxic events during ALS, but the causal relationship of most mutations and/or aggregations in these proteins and disease onset remain unclear. 


\subsection{Selective vulnerability of MNs}

The only known common denominator in ALS is the vulnerability and the loss of MNs. Whereas other neuron types and non-neuronal cells are not affected, the MN seems to be selectively vulnerable towards degeneration. For instance, the large soma size, high metabolic rates, low calcium buffering capability, high content of neurofilament and susceptibility towards mitochondrial dysfunction have been implicated in selective MN vulnerability (Shaw, 2005) (Fig.5). The low calcium buffering capability of MNs appear to result from the lack of the calcium binding proteins calbindin and parvalbumnin, whereas MNs have a high number of calcium permeable AMPA glutamate receptors (Shaw, 2005). In most MNs, AMPA receptors are missing the GluR2 subunit, which acts as an inhibitor of calcium permeability resulting in a fast calcium influx (Van Damme et al., 2002). Among other mechanisms, MNs strongly rely on mitochondria for calcium buffering, which could explain the high MN susceptibility towards mitochondrial dysfunctions (Carriedo et al., 2000). 


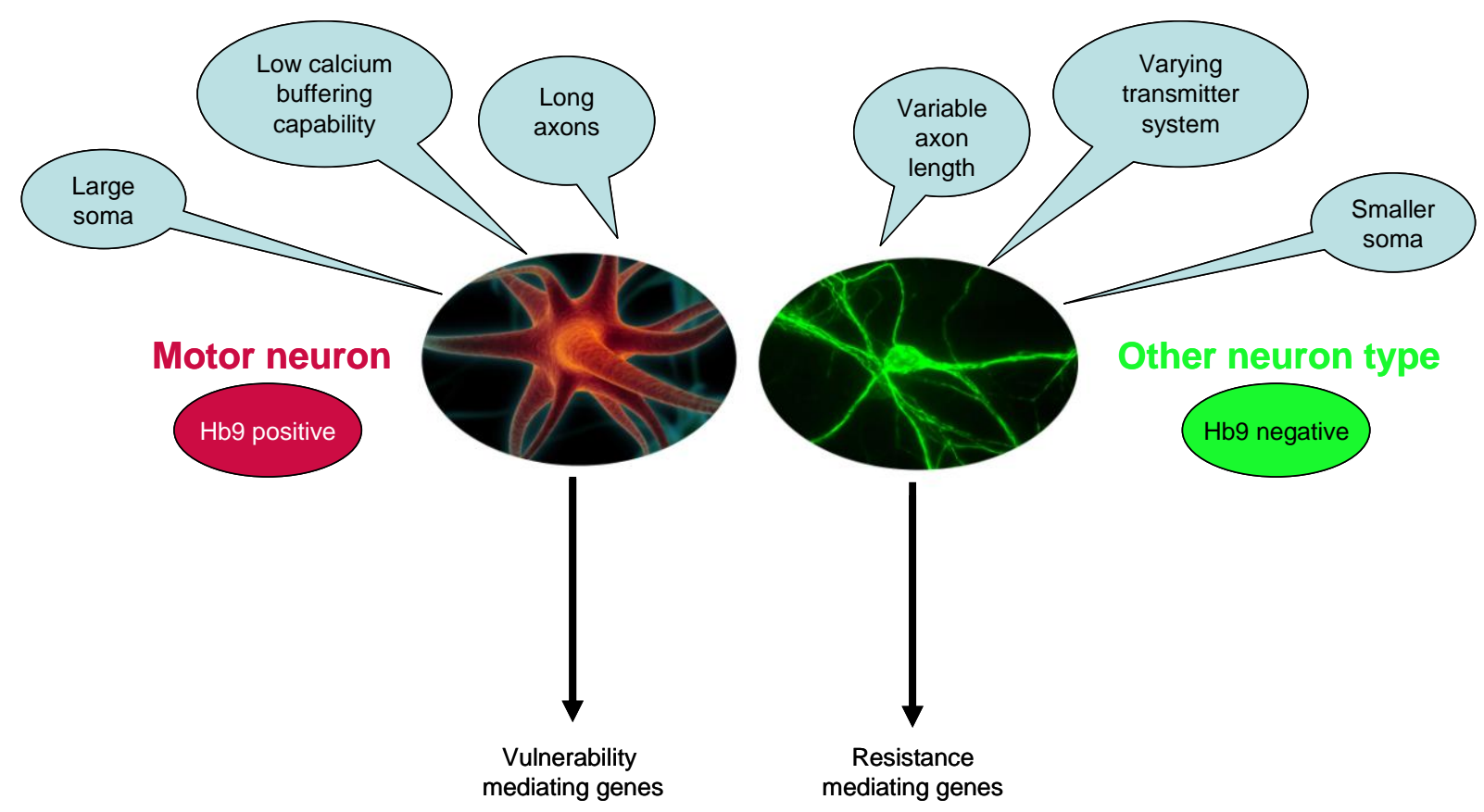

Fig. 5 Comparison of MN versus other neuron type

MNs differ in various points from other neurons, morphological as well as on molecular levels. Compared to other neurons, MNs have larger cell soma and longer axons, low calcium buffering capability, are positive for $\mathrm{Hb} 9$ and carry vulnerability mediating genes.

However, it essentially remains unclear what renders MNs, but not other neuron or non-neuronal cell types, particularly vulnerable towards degeneration in ALS.

\subsubsection{MN subtype specific vulnerability}

Interestingly, distinct MN subtypes display dramatic differences in their vulnerability towards degeneration in ALS. For instance, most ALS patients retain voluntary control over bladder and genital functions, as well as eye movements, reflecting the selective survival of the $\mathrm{MN}$ subtypes supplying the corresponding muscles (Mitsumoto, 1998). These observations are reflected by the selective survival observed in MNs controlling the urethral sphincter (UC) and bulbocavernosus (BCV) muscle in mouse ALS models (Hamson et al., 2002). In contrast, fast fatigable (FF, phasic) MNs appear to represent the most vulnerable MN subtype, as these MNs are the earliest to degenerate in ALS, while slow (S, tonic) MNs seem to display relative 
(partial) resistance, and begin to be affected only in late stages of disease progression (Pun et al., 2006). Whereas, ER-stress in fALS-linked SOD1 mutant mice seem to be selectively prone to vulnerable MNs, ubiquitin accumulations have been found in all MN subtypes (Saxena et al., 2009). Even before the identity of these fast (vulnerable) and slow (resistant) MNs was known, differences in the susceptibility of MN subtypes were found. Schaefer et al. showed that dying back of axons was for "loosers" (fast MNs), whereas "compensators" (slow MNs) could compensate effects of the neuropathology in SOD1 mutant mice (Schaefer et al., 2005). Findings of vulnerability and diversity in plasticity of distinct MN subtypes are not only restricted to disease models. In aged WT mice has been seen, that loss and weakening of synapses was increased in fast muscles. Similar results of affected plasticity and loss synapses were found in other MNDs mice models like progressive motoneuropathy (PMN) and motoneuron degeneration. Both diseases possess the hallmark of progressive MN loss (Frey et al., 2000).

These observations therefore strongly indicate intrinsic differences between distinct types of MNs that render them more or less sensitive or resistant towards neurodegeneration. It is therefore expected that among the pool of genes selectively expressed in BCV and type S MNs, for instance, will be factors involved in the intrinsic resistance of these MN subtypes. Alternatively, the specific expression of certain factors in vulnerable FF MNs may render these neurons particularly receptive for ALS-causing effectors.

\subsection{Aim of the study}

This thesis aimed to identify novel genetic factors responsible for relative vulnerability of different MN types towards motor neuron degeneration in ALS. The differential expression of genes was analyzed by a transcriptome-wide screen of three motor 
neuron subtypes which differ in their vulnerability during ALS: the fast fatigable (type FF) Rectus femoris (vulnerable), the Soleus, slow twitch (semi-resistant) and Bulbocavernosus, somatic genital (resistant). In silico analysis of the candidate genes gave insights into possible functional networks underlying the vulnerability of $\mathrm{MN}$ subtypes. Subsequently, highly differentially expressed genes were confirmed as markers for MN subtypes in vivo. The genes were screened for modifier activity in three independent neurodegenerative disease paradigms in Drosophila disease model. This narrowed down the number of possible candidate genes linked to MN vulnerability versus resistance.

Further, an ALS-linked TDP-43 chick model system was established, which allows stable overexpression of candidate genes in chick spinal cord MNs, thus enabling a functional analysis of candidate gene impact on MN survival in vivo.

Ultimately this work provided the basis to identify the mediators of $\mathrm{MN}$ vulnerability/resistance and help deepen our understanding of the mechanisms in ALS and reveal possible therapeutic targets for influencing disease progression in ALS and other MNDs. 


\section{Materials and Methods}

\subsection{Animals}

All experiments with mice were performed according to the federal law and were approved by the animal care committee of the Bezirksregierung Braunschweig, Germany. Breeding and housing took place in the animal facility of the ENI-Göttingen under the control of a veterinarian.

\subsubsection{Enzymes}

\begin{tabular}{|l|l|}
\hline Enzymes & Supplier \\
\hline Green Taq DNA Polymerase & Fermentas GmbH \\
\hline Phusion High fidelity DNA & New England Biolabs \\
Polymerase & GmbH \\
\hline Restriction Enzymes + Buffers & Fermentas GmbH \\
\hline $\begin{array}{l}\text { Shrimp alkaline phosphatase + } \\
\text { Buffer }\end{array}$ & Fermentas GmbH \\
\hline SP6 RNA Polymerase + Buffer & Roche Diagnostics GmbH \\
\hline T4 DNA ligase + Buffer & Fermentas GmbH \\
\hline T7 RNA Polymerase + Buffer & Roche Diagnostics GmbH \\
\hline
\end{tabular}

\subsubsection{General equipment}

General laboratory materials were supplied by Eppendorf (Hamburg), Starlab GmBH (Ahrensburg) and Sarstedt AG (Nürnbrecht).

\begin{tabular}{|l|l|}
\hline Equipment & Supplier \\
\hline LightCycler 480 & Roche \\
\hline Arcturus Veritas micro dissection system & $\begin{array}{l}\text { Moleculare } \\
\text { Devices }\end{array}$ \\
\hline Bioanalyzer2000 & Agilent \\
\hline Leica DMRIE2 & Leica \\
\hline Olympus IXS1 & Olympus \\
\hline
\end{tabular}




\subsubsection{Kits}

\begin{tabular}{|l|l|}
\hline Kit & Supplier \\
\hline FL-Ovation cDNA Biotin Module V2 & NuGen \\
\hline GeneChip WT sense Target Labeling Kit & Affymetrix \\
\hline QIAfilter Midi und Maxi Kit & Qiagen \\
\hline Qiaprep Spin Miniprep Kit & Qiagen \\
\hline QiaQuick Gel extraction Kit & Qiagen \\
\hline Rneasy Micro Kit & Qiagen \\
\hline TA-Cloning Kit & Invitrogen GmbH \\
\hline $\begin{array}{l}\text { WT-Ovation Pico RNA Amplification } \\
\text { System V1 }\end{array}$ & NuGen \\
\hline
\end{tabular}

\subsubsection{Microarray}

\begin{tabular}{|l|l|}
\hline Microarray & Supplier \\
\hline Mouse Gene 1.0 ST & Affymetrix \\
\hline
\end{tabular}

\subsubsection{Chemicals/General reagents}

\begin{tabular}{|l|l|}
\hline Chemicals/General reagents & Supplier \\
\hline Agar-Agar & Carl Roth $\mathrm{GmbH}$ \\
\hline Agarose NEEO & Carl Roth $\mathrm{GmbH}$ \\
\hline BSA & Carl Roth $\mathrm{GmbH}$ \\
\hline $\begin{array}{l}\text { Cholera toxin subunit B Alexa } \\
\text { conjugated }\end{array}$ & Invitrogen $\mathrm{GmbH}$ \\
\hline Diethylpyrocarbonate & Carl Roth $\mathrm{GmbH}$ \\
\hline DIG RNA labelling mix & $\begin{array}{l}\text { Roche Diagnostics } \\
\text { GmbH }\end{array}$ \\
\hline DNA Ladder & Fermentas $\mathrm{GmbH}$ \\
\hline dNTP mix & Fermentas $\mathrm{GmbH}$ \\
\hline Ethanol 99.9\% & Carl Roth $\mathrm{GmbH}$ \\
\hline Ethidium bromide & Carl Roth $\mathrm{GmbH}$ \\
\hline Formamide & Applichem $\mathrm{GmbH}$ \\
\hline GeneRuler 1 kb DNA ladder & Fermentas GmbH \\
\hline Glycerol & Carl Roth $\mathrm{GmbH}$ \\
\hline HCl & Carl Roth $\mathrm{GmbH}$ \\
\hline Heparin & Applichem $\mathrm{GmbH}$ \\
\hline Histoacryl tissue glue & B. Braun $\mathrm{GmbH}$ \\
\hline L-15 medium & Applichem $\mathrm{GmbH}$ \\
\hline Maleic acid & Carl Roth $\mathrm{GmbH}$ \\
\hline Methanol & Carl Roth $\mathrm{GmbH}$ \\
\hline MgCl2 & Carl Roth GmbH \\
\hline NaCl & Carl Roth GmbH \\
\hline NBT/BCIP tablets & Roche Diagnostics \\
\hline & \\
\hline &
\end{tabular}




\begin{tabular}{|l|l|}
\hline & GmbH \\
\hline OCT & Sakura Finetek GmbH \\
\hline Orange G & Applichem $\mathrm{GmbH}$ \\
\hline PBS pH 7.2 & Invitrogen $\mathrm{GmbH}$ \\
\hline Proteinase K & Applichem $\mathrm{GmbH}$ \\
\hline Salmon sperm DNA & Applichem $\mathrm{GmbH}$ \\
\hline SDS & Carl Roth $\mathrm{GmbH}$ \\
\hline Sodium chloride & Carl Roth $\mathrm{GmbH}$ \\
\hline Sodium citrate & Carl Roth $\mathrm{GmbH}$ \\
\hline Sucrose & Carl Roth $\mathrm{GmbH}$ \\
\hline TRIS & Carl Roth $\mathrm{GmbH}$ \\
\hline Triton X-100 & Carl Roth $\mathrm{GmbH}$ \\
\hline Trizol reagent & Invitrogen GmbH \\
\hline Tween 20 & Carl Roth GmbH \\
\hline Universal probe library (Mouse) & $\begin{array}{l}\text { Roche Diagnostics } \\
\text { GmbH }\end{array}$ \\
\hline VectaShield & Vector Labs Inc. \\
\hline Yeast extract & Applichem GmbH \\
\hline
\end{tabular}

\subsubsection{Software}

\begin{tabular}{|l|l|}
\hline Software & Supplier \\
\hline $\begin{array}{l}\text { Acrobat Reader } \\
\text { Professional 8.0 }\end{array}$ & Adobe \\
\hline BioEdit & Ibis Therapeutics \\
\hline Creative Suite CS3 & Adobe \\
\hline Endnote X2 & Thomson Research Soft \\
\hline ImageJ & http://rsbweb.nih.gov/ij/index.htm \\
\hline IPA & Ingenuity Systems \\
\hline Microsoft Office 2003 & Microsoft \\
\hline PDraw & Acalone \\
\hline Photoshop & Adobe \\
\hline Prism & Graph Pad \\
\hline Windows 7 & Microsoft \\
\hline $\begin{array}{l}\text { Partek Genomic Suite } \\
\text { v6.4 }\end{array}$ & www.partek.com \\
\hline
\end{tabular}

\subsubsection{Solutions/ Buffers}

\begin{tabular}{|l|l|}
\hline Solution & Content \\
\hline Blocking buffer & Roche blocking reagent $1 \%(\mathrm{w} / \mathrm{v})$ in PBT \\
\hline DNA running buffer & $8 \%$ Sucrose in TAE; $1 \mathrm{mg} / \mathrm{ml}$ Orange G \\
\hline Immunostaining solution & PBS pH7.2; $1 \%$ BSA; $1 \%$ Triton X-100 \\
\hline $\begin{array}{l}\text { In-Situ hybridization } \\
\text { buffer }\end{array}$ & $\begin{array}{l}50 \% \text { Formamide; } 5 \times \text { SSC pH4.5; } 1 \% \text { SDS; } 10 \mathrm{mg} / \mathrm{ml} \\
\text { Heparin; } 10 \mathrm{mg} / \mathrm{ml} \text { salmon sperm DNA }\end{array}$ \\
\hline
\end{tabular}




\begin{tabular}{|c|c|}
\hline In-Situ wash Buffer I & $50 \%$ Formamide; $5 x$ SSC pH $4.5 ; 1 \%$ SDS \\
\hline In-Situ wash Buffer II & $50 \%$ Formamide; 2x SSC pH4.5; 0.1\% Tween-20 \\
\hline LB-Agar & LB medium with $1 \%(w / v)$ agar-agar \\
\hline LB-Medium & $5 \mathrm{~g} / \mathrm{l} \mathrm{NaCl} ; 5 \mathrm{~g} / \mathrm{l}$ yeast extract; $10 \mathrm{~g} / \mathrm{l}$ tryptone \\
\hline MBST & $0.1 \mathrm{M}$ Maleic acid; $0.15 \mathrm{M} \mathrm{NaCl} \mathrm{pH} 7.5$ \\
\hline NTMT & $\begin{array}{l}\text { 100mM Sodium chloride; } 100 \mathrm{mM} \text { Tris-HCl; } 50 \mathrm{mM} \mathrm{MgCl} \text {; } \\
0.1 \% \text { Tween-20; pH9.5 }\end{array}$ \\
\hline PBT & PBS pH7.2; 0.1\% Tween-20 \\
\hline SSC 20x stock solution & 3M NaCl; 300mM Sodium citrate; pH4.5 \\
\hline $\begin{array}{l}\text { TAE buffer } 50 x \text { stock } \\
\text { solution, } 1000 \mathrm{ml}\end{array}$ & $\begin{array}{l}2 \mathrm{M} \text { Tris-Acetate, } \mathrm{pH} 8.0 ; 50 \mathrm{mM} \text { EDTA; } 57.1 \mathrm{ml} \text { Glacial } \\
\text { acetic acid; Add dH2O up to } 1000 \mathrm{ml}\end{array}$ \\
\hline Tail biopsy lysis bi & $\begin{array}{l}0.1 \mathrm{M} \text { Tris pH } 8 ; 30 \mathrm{mM} \mathrm{NaCl} ; 0.2 \mathrm{mM} \text { EDTA; } 0.05 \text { SDS; } \\
0.5 \mathrm{mg} / \mathrm{ml} \text { Proteinase K }\end{array}$ \\
\hline
\end{tabular}

\subsubsection{Vectors}

\begin{tabular}{|l|l|}
\hline Vector & Supplier \\
\hline pCrII-TOPO & Invitrogen $\mathrm{GmbH}$ \\
\hline pCAGEN & Addgene \\
\hline pGK:Cre & Addgene \\
\hline Hb9:Cre & Lee et al.,2004 \\
\hline
\end{tabular}

\subsection{Mouse line}

The mouse line used for these experiments is an $H b 9:: G F P$ transgenic line, expressing GFP under the control of a $\mathrm{Hb}$ 9 promotor in postmitotic motor neurons (Lee et al., 2004).

\subsubsection{Genotyping of the Hb9::GFP mouse line}

Mouse tissue derived from tail biopsies was lysed in tail biopsy lysis buffer over night at $56^{\circ} \mathrm{C}$ followed by centrifugation at $16.000 \times \mathrm{g}$ for 10 minutes. The supernatant was transferred into a tube and mixed with $500 \mu \mathrm{l}$ of isopropanol. After centrifugation for 2 minutes at $16.000 \times \mathrm{g}$, all liquid was removed and the pellet was washed with $500 \mu \mathrm{l}$ of $70 \%$ Ethanol. Subsequent to 2 minutes of centrifugation at $16.000 \times \mathrm{g}$, the Ethanol 
was removed and the pellet air-dried. The DNA pellet was resuspended in $50 \mu$ l of water.

Genotyping was performed as a PCR with the following components:

$3 \mu \mathrm{l} \quad$ Green Taq reaction buffer

$2 \mu \mathrm{l} \quad 25 \mathrm{mM} \mathrm{MgCl}_{2}$

$1 \mu \mathrm{l} \quad$ Mouse tail DNA

$1 \mu \mathrm{l} 10 \mathrm{mM}$ EGFP forward primer (CCT ACG GCG TGC AGT GCT TCA GC)

$1 \mu \mathrm{l} 10 \mathrm{mM}$ EGFP reverse primer (CGG CGA GCT GCA CGC TGC GTC CTC)

$0.5 \mu \mathrm{l} 10 \mathrm{mM}$ dNTP mix

$0.5 \mu \mathrm{l}$ Green Taq DNA polymerase

adjust to a total volume of $30 \mu \mathrm{l}$ with $\mathrm{ddH}_{2} \mathrm{O}$

\subsubsection{Retrograde tracing of motor neurons}

$\mathrm{P} 18 \mathrm{Hb} 9$ positive male mice were anaesthetised with Ketamin/Xylen in a dosage of $100 \mu \mathrm{l} / 20 \mathrm{~g}$ body weight. The areas around the muscles of interest were shaved and cleaned with $70 \%$ ethanol. Incisions were done with micro scissors at appropriate positions for injections into A) the Rectus femoris, B) the Soleus and C) the Bulbocavernosus. A glass injection capillary was filled with lyophilized Alexa conjugated Cholera Toxin subunit B and the muscles of interest were injected up to 3 times in different spots. Afterwards the wounds at the incision sites were sealed with Histoacryl tissue glue. The mice were transferred into a humidified incubation chamber set to $35^{\circ} \mathrm{C}$ until they woke up followed by to days of breading for the actual retrograde tracing.

\subsubsection{Tissue preparation for laser capture micro dissection}

Two days after injection, at the stage P20, the mice were sacrificed by cervical dislocation and the spinal cord was extracted. The spinal cord was directly transferred into ice cold PBS, afterwards embedded into OCT and flash frozen on dry ice. Samples were stored at $-80^{\circ} \mathrm{C}$ until further use. Using a Leica CM $1510 \mathrm{~S}$ 
cryostat, cryo sections were taken from the region of labelled MNs in a total thickness of $10 \mu \mathrm{m}$ and picked up on Superfrost Plus glass slides. The slides were processed immediately or stored at $-80^{\circ} \mathrm{C}$. For immediate use, the sections were fixed in $70 \%$ ethanol for 30 seconds, followed by a dehydration row of two times $95 \%$ ethanol for 30 seconds, two times fresh $100 \%$ ethanol for one minute and two times xylene for 2 minutes. Subsequently the slides were air dried and stored in a sterile box. Everything used in the procedure was RNase free. Processed slides were directly used for laser directed micro-capture.

Following steps of laser capture and array processing were done in collaboration with Dr. Moritz Rossner and Dr. Sven Wichert

\subsubsection{Laser capture micro-dissection}

The Alexa 555/488 positive cells were micro dissected from the ventral region of the spinal cord. HS Transfer Caps (Molecular Devices) were used to capture from 40 to 50 labelled MNs per cap from several slides of one animal. For the micro-dissection we use the Arcturus Veritas micro dissection system (Molecular Devices) with fluorescence package. The single cell capture was done by identification of labelled cells in fluorescence light. Validation of captured cell was done at the quality control stations of the system, as well in bright-field as fluorescence mode.

Once up to 50 cells were captured, the cap was removed from the device and the micro dissected cells were lysed in $100 \mu$ l of RNA lysis buffer (Qiagen) by vortexing for 30 seconds. The samples were stored at $-80^{\circ} \mathrm{C}$ until further use. All steps were carried out under RNase free conditions. 


\subsubsection{RNA purification from captured cells}

For the isolation of total RNA (100pg - 100ng) the RNeasy micro kit (Qiagen) was used according to manufacturer's protocol.

\subsubsection{Quantification of RNA}

Quality and quantity control was performed on a Nano-labchip using the Bioanalyzer2000 (Agilent) following the supplier's instructions.

\subsubsection{Amplification for microarray hybridization}

For the use of the total RNA in microarray hybridization it was necessary to amplify the samples using the WT-Ovation Pico RNA Amplification System V1 (Catalog \# 3300-12; NuGen) and to label with FL-Ovation cDNA Biotin Module V2 (Catalog \#4200-12; NuGen) following exactly the protocol provided by the supplier.

\subsubsection{Microarray hybridization}

\begin{tabular}{|l|c|}
\hline Type of array & Mouse Gene 1.0ST \\
\hline number of probes & $\mathbf{7 7 0} 317$ \\
\hline
\end{tabular}

For hybridization, minimum of $10 \mu \mathrm{g}$ of labelled cDNA was used. All steps of hybridization, washing, staining, and scanning were performed under standard conditions and followed according to the manufacturer's protocol.

\subsubsection{Microarray data analysis}

Data obtained from the array was analyzed with Partek Genomic Suite v6.4 (www.partek.com) performed by Dr. Sven Wichert according to the following protocol:

1. pre-processing of data, including probe level RMA backround correction 
2. quantile normalization across all arrays and $\log _{2}$ transformation followed by median polish in order to summarize probes and obtain the overall score for each probe set.

3. filtering of the data based on the expression values of each probe set within the replicate set for each sample (probes with an expression value less than $3.5 \log _{2}$ in all samples were removed and excluded from analysis)

4. performing of a one-way anova statistical test on normalized and filtered probe set level intensities between each group to generate $p$-values and fold-change values.

Subsequent analysis was performed with IPA (Ingenuity Systems).

\subsection{In situ probe design}

The primer sequences for the in Situ probes of the candidates derived from the micro array, Cartpt, Lifr and Lxn were taken from the Allen Brain Atlas (@2004 - 2010 Allen Institute for Brain Science). The primers for the Uts2 probe were derived from Dubessy et al.,2008 (Dubessy et al., 2008).

\begin{tabular}{|c|c|c|c|c|}
\hline $\begin{array}{c}\text { NCBI } \\
\text { Accession }\end{array}$ & $\begin{array}{c}\text { Gene } \\
\text { Symbol }\end{array}$ & Gene Name & Forward Primer & Reverse Primer \\
\hline NM_013732 & Cartpt & $\begin{array}{c}\text { cocaine and amphetamine } \\
\text { regulated transcript }\end{array}$ & GCTACCTTTGCTGGGTGC & CAACAGGGAAAGAGCCCA \\
\hline NM_016753 & Lxn & latexin & GCCGTGATCTCTTTGAACTAGG & GGAATCTATGCAGCTCGGTACT \\
\hline NM_013584 & Lifr & $\begin{array}{c}\text { leukemia inhibitory factor } \\
\text { receptor }\end{array}$ & TCTGTACGGGCAAACCGT & TGGTGATGAGCCAGCAGA \\
\hline NM_011910.2 & Uts2 & urotensin 2 & TCTGCTGCCTGCTCTTCATAGG & AAGAACAAGGCGTCGTTAGCG \\
\hline
\end{tabular}

The cDNA derived from micro capture was used for in situ probe amplification or stored at $-80^{\circ} \mathrm{C}$.

The in situ probe fragments were amplified in a PCR using the following settings:

\begin{tabular}{|l|l|l|l|}
\hline Denaturation & $2^{\prime}$ & $94^{\circ} \mathrm{C}$ & \\
\hline Denaturation & $0.10^{\prime}$ & $94^{\circ} \mathrm{C}$ & \\
\hline Annealing & $0.5^{\prime}$ & $55^{\circ} \mathrm{C}$ & 35 cycles \\
\hline Elongation & $0,5^{\prime}-1^{\prime}$ & $72^{\circ} \mathrm{C}$ & \\
\hline Elongation & $3^{\prime}$ & $72^{\circ} \mathrm{C}$ & \\
\hline End & on hold & $4^{\circ} \mathrm{C}$ & \\
\hline
\end{tabular}


The Quality of the PCR product was checked with agarose gel electrophoresis on a $1 \%$ agarose gel. Positive PCR fragments were cut out and cleaned by using QIAquick Gel Extraction Kit following the QIAGEN standard protocol.

\subsubsection{TA-Cloning}

Cleaned PCR fragments were then used for TOPO-Cloning using the TA-Cloning Kit (Invitrogen $\mathrm{GmbH}$ ) according to the manufactures protocol.

Chemical competent E.coli were transformed by adding $10 \mu \mathrm{l}$ of the TOPO reaction solution into $90 \mu \mathrm{l}$ of $E$. coli. After 30 minutes on ice the cells were heat shocked at $42^{\circ} \mathrm{C}$ for two minutes and subsequently chilled for one minute on ice. Afterwards $900 \mu \mathrm{l}$ of LB-medium were added and the cells were incubated on a shaker at $37^{\circ} \mathrm{C}$ for one hour. After the incubation, the cells were spinned down at 3500rpm for 3 minutes and plated on LB-Agar plates containing the correct antibiotic for selection. The plates were incubated over night at $37^{\circ} \mathrm{C}$.

To verify positive clones, colonies were picked with autoclaved toothpicks and transferred into $5 \mathrm{ml}$ LB-Medium containing the appropriate antibiotic. These minis were then incubated on a shaker at $37^{\circ} \mathrm{C}$ over night. The isolation of the plasmids was carried out using the QIAGEN Mini Prep Kit following the instructions of the manufactures protocol. Using the primers of the in Situ probes, a PCR was performed on these plasmid preparations. Positive minipreps were sequenced for further verification.

After the verification of the cloned in situ fragments into the PCRII-TOPO vector, the vector was linearized using the restriction enzyme fitting the orientation of the fragment. The antisense RNA probe was created using the DIG-RNA labelling mix 
and the appropriate RNA polymerase (SP6/T7) according to the orientation of the insert. The synthesis of the probe was done according to the manufacturer's protocol. Quality and quantity of RNA and DNA was measured using the Nanodrop ND-1000 UV-Vis spectrophotometer (Peqlab Biotechnologie $\mathrm{GmbH}$, Erlangen), as well as on the agarose gel.

\subsubsection{In situ Hybridization}

P20 Hb9 positive mouse spinal cord was extracted and fixed in 4\% PFA in 1x PBS for two days on a shaker at $4^{\circ} \mathrm{C}$. Fixed spinal cord were embedded into OCT. Embedded spinal cords were cut in a cryostat at $-20^{\circ} \mathrm{C}$ in $10 \mu \mathrm{m}$ thick sections and collected on Superfrost glass slides. Slides were stored a $-80^{\circ} \mathrm{C}$ or directly used for in situ hybridization.

For in situ hybridization the slides were processed as followed:

(Protocol after Jing Yu from the Andy McMahon Laboratory)

Day 1:

1. Slides were fixed in $4 \%$ PFA / PBS for 10 minutes.

2. washed in PBS 3 times for 3 minutes.

3. treated with Proteinase $\mathrm{K}(10 \mu \mathrm{g} / \mathrm{ml}$ in PBS) for 10 minutes.

4. washed 3 times in PBS for 3 minutes.

5. fixed in 4\% PFA / PBS for 5 minutes.

6. washed in PBS for 3 times for 5 minutes.

7. washed with $0.85 \% \mathrm{NaCl}$ for 3 minutes.

8. washed with $70 \% \mathrm{EtOH} / 0.85 \% \mathrm{NaCl}$ for 5 minutes.

9. washed with $95 \% \mathrm{EtOH}$ for 5 minutes.

10. air dried for 10 minutes on Wattman Paper.

11. prehybridized with prehybridization buffer for one hour at $68^{\circ} \mathrm{C}$. 
12. Probes were diluted with prehybridization buffer to a final concentration of $500 \mathrm{ng} / \mathrm{ml}$. The diluted probe was heated up for 5 minutes at $80^{\circ} \mathrm{C}$.

The slides were horizontally arranged in a box humidified with $50 \%$ formamide $/ 5 x$ SSC. Excess solution was removed and $250 \mathrm{ml}$ of prehybridization buffer containing the probe were applied. The slides were sealed with a stripe of parafilm on top of each slide and incubated over night at $68^{\circ} \mathrm{C}$.

\section{Day 2:}

1. Slides were immerse in $5 x \mathrm{SSC}$ at $68^{\circ} \mathrm{C}$ to separate from parafilm.

2. washed in $5 \times \mathrm{SSC} / 50 \%$ formamide at $65^{\circ} \mathrm{C}$ for $30 \mathrm{~min}$.

3. washed 2 times in $2 \times S S C$ for 20 minutes at $65^{\circ} \mathrm{C}$.

4. washed 2 times in $0,2 \times \mathrm{SSC}$ for 20 minutes at $65^{\circ} \mathrm{C}$.

5. washed 3 times in MBST for 5 minutes at RT.

6. incubated with $1 \mathrm{x}$ blocking buffer (BB) in MBST for 1-2 hours at RT.

7. excess solution was removed and $150 \mu$ l of antibody in a final concentration of 1:4000 in $1 \times$ BB/MBST were applied. Slides sealed with parafilm and incubated over night at $4^{\circ} \mathrm{C}$.

\section{Day 3:}

1. Slides were washed with MBST to remove the parafilm.

2. washed with MBST 3 times for 5 minutes.

3. washed with NTMT, pH9.5 for 10 minutes.

4. incubated with a tablet of NBT/BCIP solved in $20 \mathrm{ml}$ of water at RT.

Slides were incubated until a strong signal developed. Subsequently, the reaction was stopped by washing with PBS 5 times for 5 minutes. 
The slides were mounted with 50\% Glycerol/PBS and cover slipped.

Images from in situ hybridizations were taken with a Leica TCS/MP confocal/twophoton microscope.

\subsection{Quantitative real-time PCR}

The real-time PCR primers were designed in the Universal Probe Library (UPL) Assay Design Center supplied online by Roche Applied Science

(https://www.roche-applied-science.com/sis/rtpcr/upl/index.jsp?id=uplct_030000).

\begin{tabular}{|c|c|c|c|c|}
\hline $\begin{array}{c}\text { NCBI } \\
\text { Accession }\end{array}$ & $\begin{array}{c}\text { Gene } \\
\text { Symbol }\end{array}$ & Gene Name & Forward Primer & Reverse Primer \\
\hline NM_013732 & Cartpt & $\begin{array}{c}\text { cocaine and } \\
\text { amphetamine } \\
\text { regulated transcript }\end{array}$ & GCTACCTTTGCTGGGTGC & CAACAGGGAAAGAGCCCA \\
\hline NM_016753 & Lxn & latexin & GCCGTGATCTCTTTGAACTAGG & GGAATCTATGCAGCTCGGTACT \\
\hline NM_013584 & Lifr & $\begin{array}{c}\text { leukemia inhibitory } \\
\text { factor receptor }\end{array}$ & TCTGTACGGGCAAACCGT & TGGTGATGAGCCAGCAGA \\
\hline NM_011910.2 & Uts2 & urotensin 2 & TCTGCTGCCTGCTCTTCATAGG & AAGAACAAGGCGTCGTTAGCG \\
\hline NM_011654.2 & Tuba1b & alpha Tubulin 1B & AGGAGCTGGCAAGCATGT & AGCTGCTCAGGATGGAAGAG \\
\hline
\end{tabular}

Experiments were performed using a Roche LightCycler 480 system and the universal library probe. Expression was normalized to alpha Tubulin 1B. All steps followed the Roche LightCycler protocol.

\subsection{Long-term expression system in Chick embryo}

We established a genome integrating long-term expression construct, in order to diminish effects of dilution and therefore getting a strong expression without variation. We started of with a specific expression of candidate genes in MNs driven by a strong pCAGGS promoter and co-electroporation of a Hb9::Cre vector. The Hb9::Cre vector works on basis of a Cre recombinase recognizing loxP sites flanking a termination signal of three beta-globin polyadenylation signals (Hoess et al., 1984) and therefore allowing transcription start by excision of the stop signal. Nevertheless, 
over time the electroporated expression constructs were diluted or degraded and the expression level was decreased.

In order to eliminate this effect, we designed an expression construct, which integrates into the genome of the electroporated cell via a tol2 transposase (Kawakami and Shima, 1999). The transposase recognizes the tol2 sites flanking the pCAGGS construct and intergrates the fragment into the genome of the electroporated chick embryo (Sato et al., 2007; Takahashi et al., 2008). Combined with the Hb9::Cre vector we obtained a strong, specific and stable long-term expression in MNs of chick embryo spinal cord.

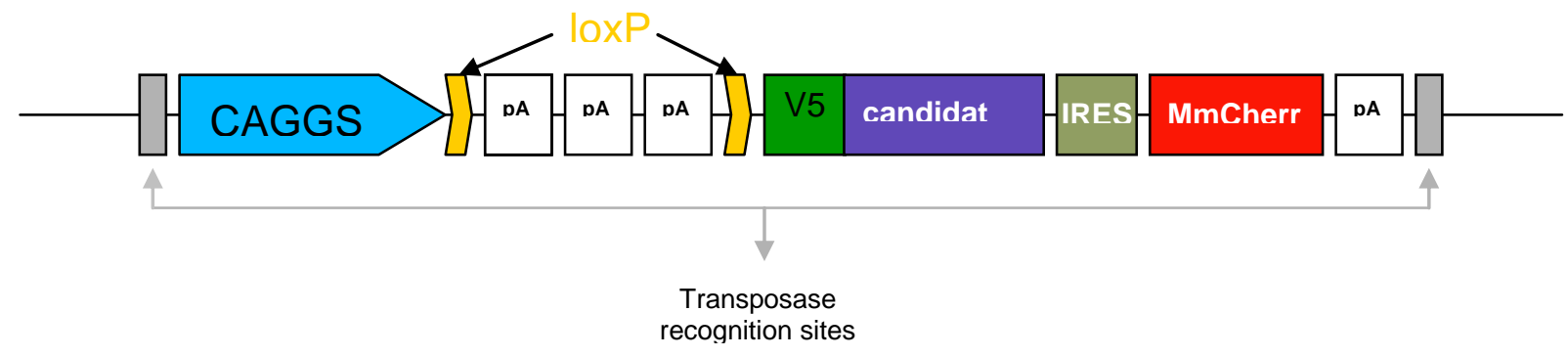

\section{Long-term expression vector (LTEV)}

A strong PCAGGS promoter is followed by three floxed polyA signals, V5 tag, IRES site, a reporter Mmcherry and a terminal polyA signal, all together flanked by transposase recognition sites.

Besides a Mmcherry (Shaner et al., 2004) serving as a reporter for electroporation efficiency, a V5 tag enables visualization of the candidate protein expression via immonostaining.

\subsubsection{Chick expression constructs}

The Hb9::Cre vector was a gift from S. Pfaff. The pCAGGS::T2TP vector (Kawakami and Noda, 2004) was a gift from K. Kawakami. The expression vector consisted of a pCAGGS promoter derived from the pCAGEN vector (Matsuda and Cepko, 2004), followed by three $S V 40 p A$ signals flanked by two loxP sites, V5-tag TDP-43 expression cassette, IRES (Internal ribosome entry site), MmCherry open reading 
frame and a terminating $S V 40 \mathrm{pA}$ signal, and were inserted between the Tol2-sites as a 5'Ssp // 3'Xho / fragment. The Tol2 vector (Urasaki et al., 2006) was a gift from A. Urasaki. The TDP-43 variants were cloned after PCR amplification in frame between the V5-tag and the IRES as a 5'Xbal / 3'Smal fragment using the following primers:

\begin{tabular}{|l|l|}
\hline Description & \multicolumn{1}{c|}{ Sequence } \\
\hline TDP-43 forward/Xba I & 5' GCTCTAGAATGTCTGAATATATTCGGGTAACC 3' \\
\hline TDP-43 reverse/Sma I & 5' TCCCCCGGGCTACATTCCCCAGCCAGAAGACTT 3' \\
\hline TDP-43 CTF forward/Xba I & 5' GCTCTAGACTGCGGGAGTTCTTCT 3' \\
\hline TDP-43 NTF reverse/Sma I & 5'CCCGGGGGACTATTAAGCATCTGTCTCATCCATTT 3' \\
\hline
\end{tabular}

All TDP-43 constructs were verified by complete sequencing.

\subsubsection{In ovo electroporation}

Fertilized chicken (Gallus domesticus) eggs were obtained from Geflügelzucht Horstmann $\mathrm{GmbH}$. These eggs were incubated at $37.5^{\circ} \mathrm{C}$ and $80 \%$ humidity (J. Hemel-Brutgeräte $\mathrm{GmbH}$ u. Co KG) for 72 hours ( 3 days) to obtain embryos that are at the developmental stage E3. Microinjections were performed using a micropipette needle made from pulled glass capillary tubes. The needle was loaded with a mixture of DNA, Hb9::Cre (with concentration of $1.0 \mu \mathrm{g} / \mu \mathrm{l})$ for $\mathrm{MN}$ specific expression, pCAGGS::T2TP for long-term-expression (concentration of $1.0 \mu \mathrm{g} / \mu \mathrm{l}$ ) and the LTEV containing the TDP-43 variant (concentration of $1.0 \mu \mathrm{g} / \mu \mathrm{l}$ ). Up to $5 \mathrm{ml}$ of egg white was sucked out by poking a hole into the thinner end of the egg by the use of a syringe. Afterwards the egg was windowed in the middle. Egg yolk and the embryo stayed unharmed during this procedure. The DNA-mixture was injected into the neural tube (lumbar region) of the embryo. The injection site was electroporated using an ECM 830 electroporation system (BTX Harvard Apparatus, Holliston, USA). The electrodes were placed in parallel so that the developing spinal cord was 
situated between the electrodes. Electroporation with the electrodes placed in this way transported DNA located in the spinal cord towards the positive electrode, resulting in approximately half of the spinal cord being transfected. The electroporation settings were 5 pulses of $25 \mathrm{mV}$ for $50 \mathrm{~ms}$ in the LV $99 \mathrm{~ms} / 500 \mathrm{~V}$ modus. After electroporation, the window on the operated egg was sealed with clear sellotape, and the egg was returned to the incubator.

\subsubsection{Chick embryo and tissue processing}

Chick embryos were harvested 2 and 6 days after electroporation, and placed in cold 1x PBS. Spinal cords were dissected at embryonic day 9 (E8), while E5 spinal cords were left intact in the embryo to minimize damage. Tissues were fixed by immersion in 4\% paraformaldahyde (in 1x PBS) (1.5-2.5 $\mathrm{h}$ for E5, 4-6 $\mathrm{h}$ for E9) and then washed in 1x PBS overnight. Afterwards the tissue was incubated in $30 \%$ sucrose (in 1x PBS) overnight. All previous steps were taken out at $4^{\circ} \mathrm{C}$. Ready fixed tissue was imbedded into OCT (Jung, Nussloch). Transversal cryosections of the spinal cord were cut in a CM1900 cryostat (Leica, Bensheim) at $-20^{\circ} \mathrm{C}$ and placed on a super frost glass slide.

\subsubsection{Immunohistochemistry}

Immunoflourescent staining was performed on $30 \mu \mathrm{m}$ slices of prefixed E5 and E9 chick embryo spinal cord. For primary antibody detection the slides were incubated in a humidified box over night at $4^{\circ} \mathrm{C}$, utilizing $1 \% \mathrm{BSA}$ (fraction V) in $1 \times$ PBS with $0.5 \%$ TritonX-100 to enhance tissue penetration. Slides were washed with 1x PBS before the secondary staining, that was carried out with the same detergent for $30 \mathrm{~min}-1 \mathrm{~h}$ at RT. Slides were washed again with $1 \mathrm{x}$ PBS and stained with DAPI (Roth, 
1:1000000 w/v) at RT for 10-20 minutes. After DAPI staining, the slides were washed with 1x PBS, mounted with 50\% glycerol in PBS and cover slipped.

Primary antibodies: rabbit anti Isl $1 \frac{1}{2}$ (K5, 1:2500, gift from S.L.Pfaff), mouse anti Isl $1 / 2$ (DSHB, 1:200), mouse anti V5 (Invitrogen, 1:500). Secondary antibodies: Alexa 647 rabbit anti mouse (Invitrogen, 1:1000), Alexa 488 mouse anti rabbit (Invitrogen, 1:1000), Alexa 647 mouse anti rabbit (Invitrogen, 1:1000), Alexa 488 rabbit anti mouse (Invitrogen, 1:1000).

\subsubsection{Microscopy and image analysis}

All images of mounted slices were collected using a Leica TCS/MP confocal/twophoton microscope. All images were acquired from $30 \mu \mathrm{m}$ sections of the chick embryo spinal cord at the developmental stages of E5 and E9. Images of the E9 sections were acquired by using the 20x objective with $2 x$ zoom. Images of the E5 sections were acquired with the 40x objective and 2x zoom. For both developmental stages $(E 5, E 9$,$) all images were a total of a 20-30 \mu \mathrm{m}$ stack of $10 \mathrm{z}$-sections subsequently collapsed to a $2 \mathrm{D}$ rendering using ImageJ. MN survival was measured by counting Is| $1 / 2$ positive cells in the ventral horn, electroporated vs. non electroporated side. The counting was performed in ImageJ. 


\section{Results}

Amyotrophic lateral sclerosis (ALS) is a fatal disease which leads to a progressive motor neuron (MN) loss. Due to the $\mathrm{MN}$ loss, the muscle fibers lose their innervations, which leads to paralysis (Mulder, 1982). Interestingly, some MN subtypes display dramatic differences in their vulnerability towards the disease (Hamson et al., 2002; Mitsumoto, 1998; Pun et al., 2006).

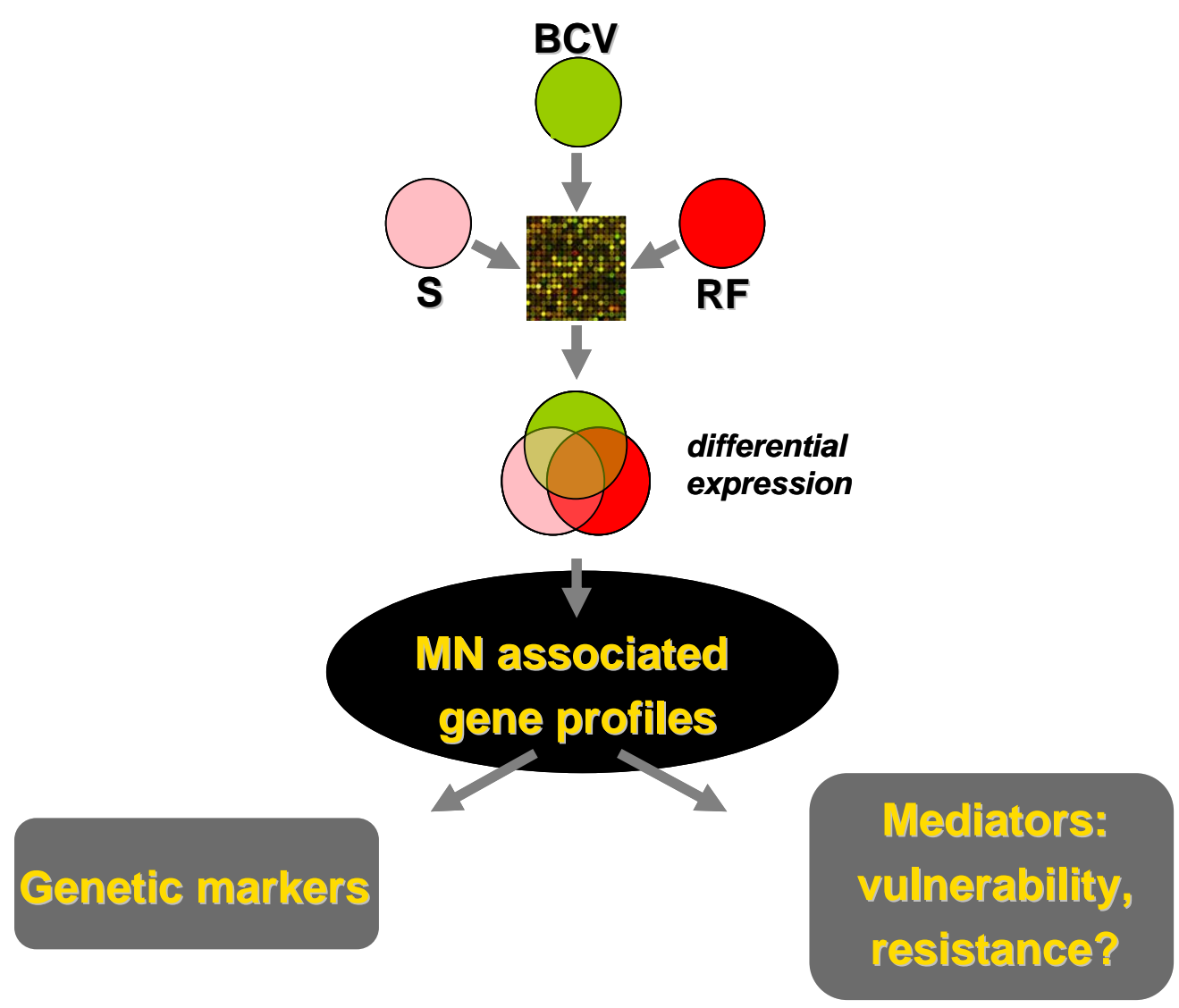

Fig. 6 Differential screen of MN subtype specific gene expression

Transcriptome screen of the Rectus femoris-, Soleus- and Bulbocavernosus MNs. Differential expression is detected performing an array, revealing genetic markers and mediators of vulnerability/resistance towards MNDs of MN subtypes. 
In order to understand what renders different motor neuron subtypes vulnerable or resistant to MNDs, the aim was to identify novel genetic factors linked to these different MN types. Because of the absence of available markers for different MN subtypes, these MNs were retrogradly labelled through intramuscular injection of Cholera toxin subunit B (CTXB). Three muscles were injected to lable type FF (vulnerable), type S (relatively resistant) and genital (resistant) MNs, respectively: the M. Rectus femoris $(R F)$, the $M$. Soleus $(S)$ and the M. Bulbocavernosus (BCV). Retrograde axonal transport of the CTxB allows specific labelling of the MNs innervating the injected muscle via Alexa dye conjugated to the CTxB. The transgenic mouse line L2 was used, expressing eGFP in postmitotic motor neurons under the control of the Hb9 promotor. Thus, the Hb9::eGFP mouse line allows the identification of labelled cells as MNs. Fig. 7 shows the extracted spinal cord of a L2 mouse at the postnatal stage $4(\mathrm{P} 4)$ with a retrogradly traced $B C V$ MNs. The $B C V$ is a triangular shaped muscle between the rectum and the penis (Fig. 7C) and it is innervated by MNs located medially in the lower lumbar region of the spinal cord. Fig. $7 A$ shows $M N s$ innervating the $B C V$ in red. The $B C V$ innervated $\mathrm{CTxB}^{+}$labelled cells were further shown to be $H b 9:: e G F P^{+}$MNs (Fig. 7B). Due to the unilateral injection of the CTxB tracer, the MNs are only labelled in one half of the spinal cord (Fig. 7A, C). 
A

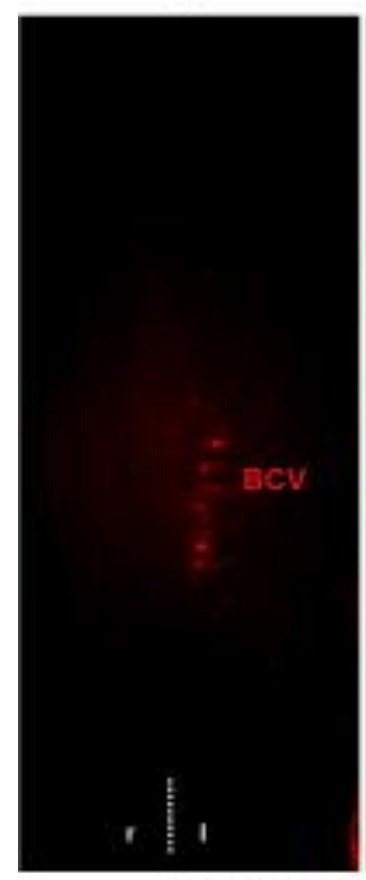

spinal cord: ventral view
B

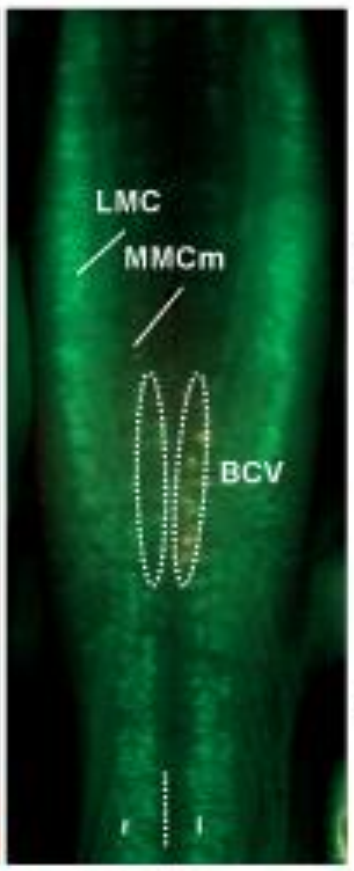

sacral

\section{Unilateral intramuscular injection:} bulbocavernosus

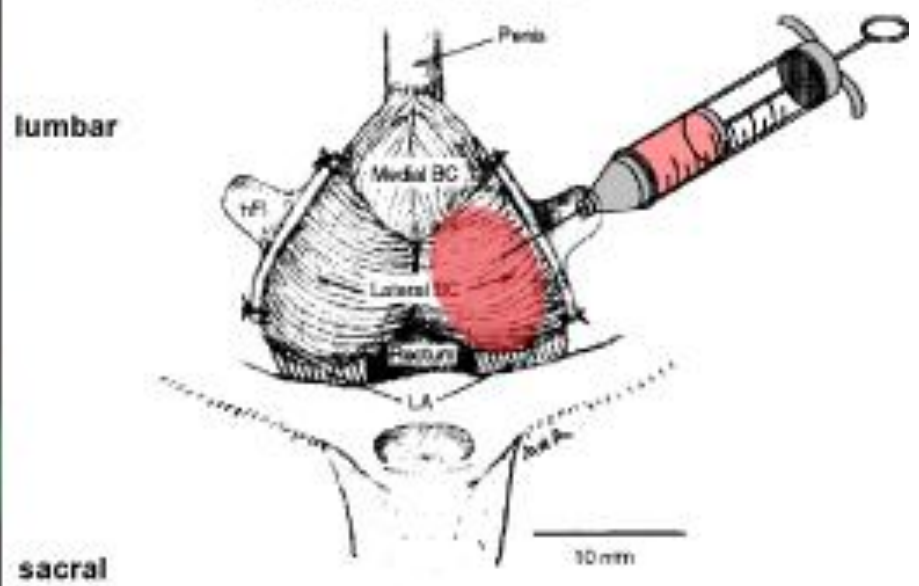

Hb9::eGFP transgene:

somatic motor neurons

Fig. 7 Retrograde tracing of MNs

(A) P4 stage spinal cord of Hb9::GFP transgenic mouse; unilateral traced MNs of the $B C V$ muscle in red. (B) Overlay of $\mathrm{Hb9}$ positive MNs in green with traced MNs in red. (C) Scheme of $B C V$ anatomy, showing the injection site (adopted from Rand and Breedlove.,1992).

We used the P4 stage to visualize the $H b 9$ positive $\mathrm{MNs}$ and the retrograde tracing in a whole mount of the spinal cord, since in later stages the signal of the eGFP positive MNs and the retrograde tracing signal is diminished by the increased thickness of more mature spinal cord, and the Hb9::eGFP signal is gradually lost due to the weaker $\mathrm{Hb9}$ expression in mature MNs (Fig. 8). 

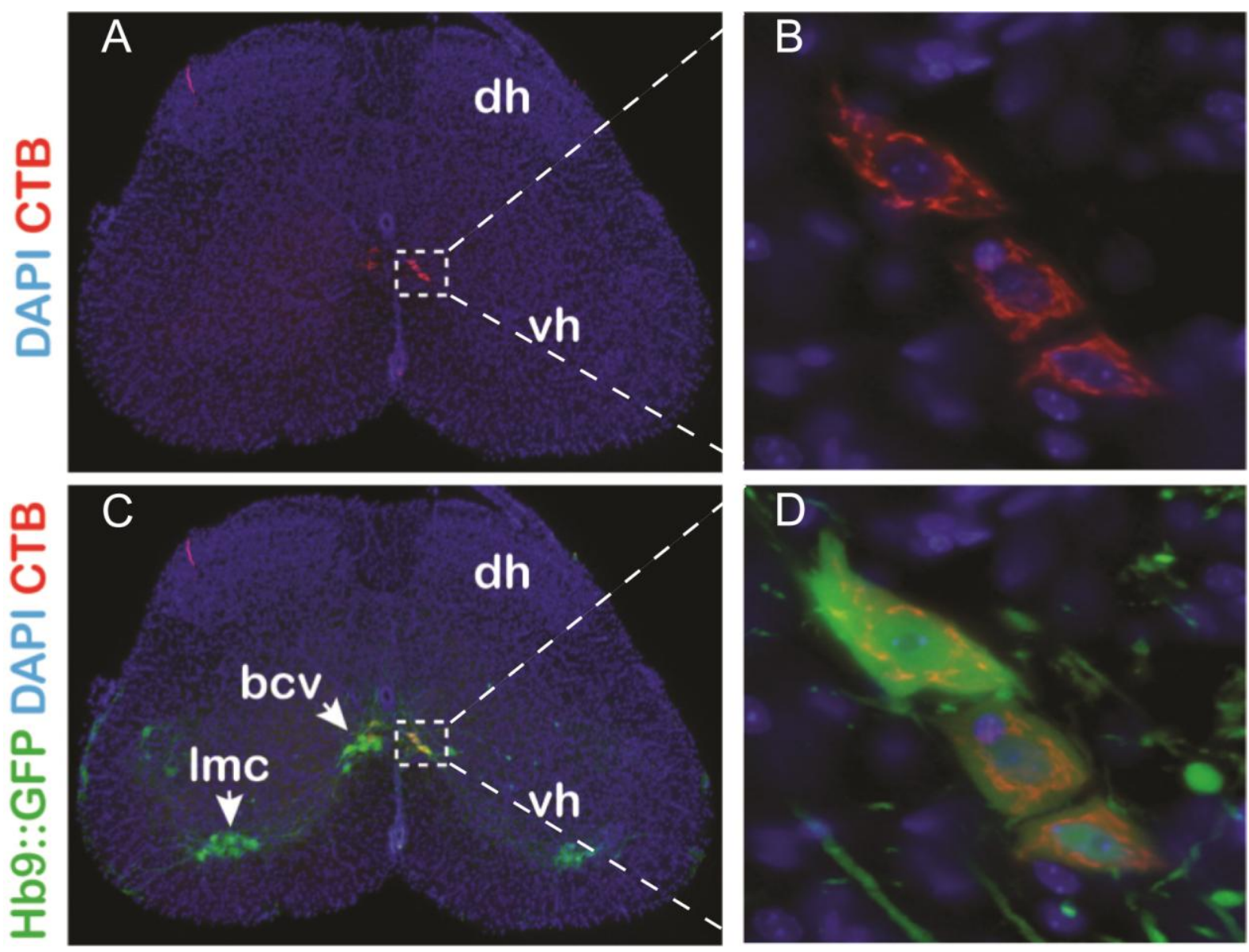

\section{Fig. 8 Cholera toxin traced MNs}

(A) Cholera toxin subunit $B(C T \times B)$ traced neurons overlap with Hb9::GFP as a marker for MNs in green (C). (B, D) Magnifications of marked zones in A and C. DAPI was used as a nuclear marker shown in blue.

Subsequently, P20 stage juvenile male mice were used, whereas male mice have a bigger $B C V$ that is easier to access. Overlap of traced neurons with $\mathrm{Hb} 9$ as a marker for MNs was shown in 10um vertical section of P20 mouse spinal cord stained with a anti-GFP antibody (Fig. 8). 


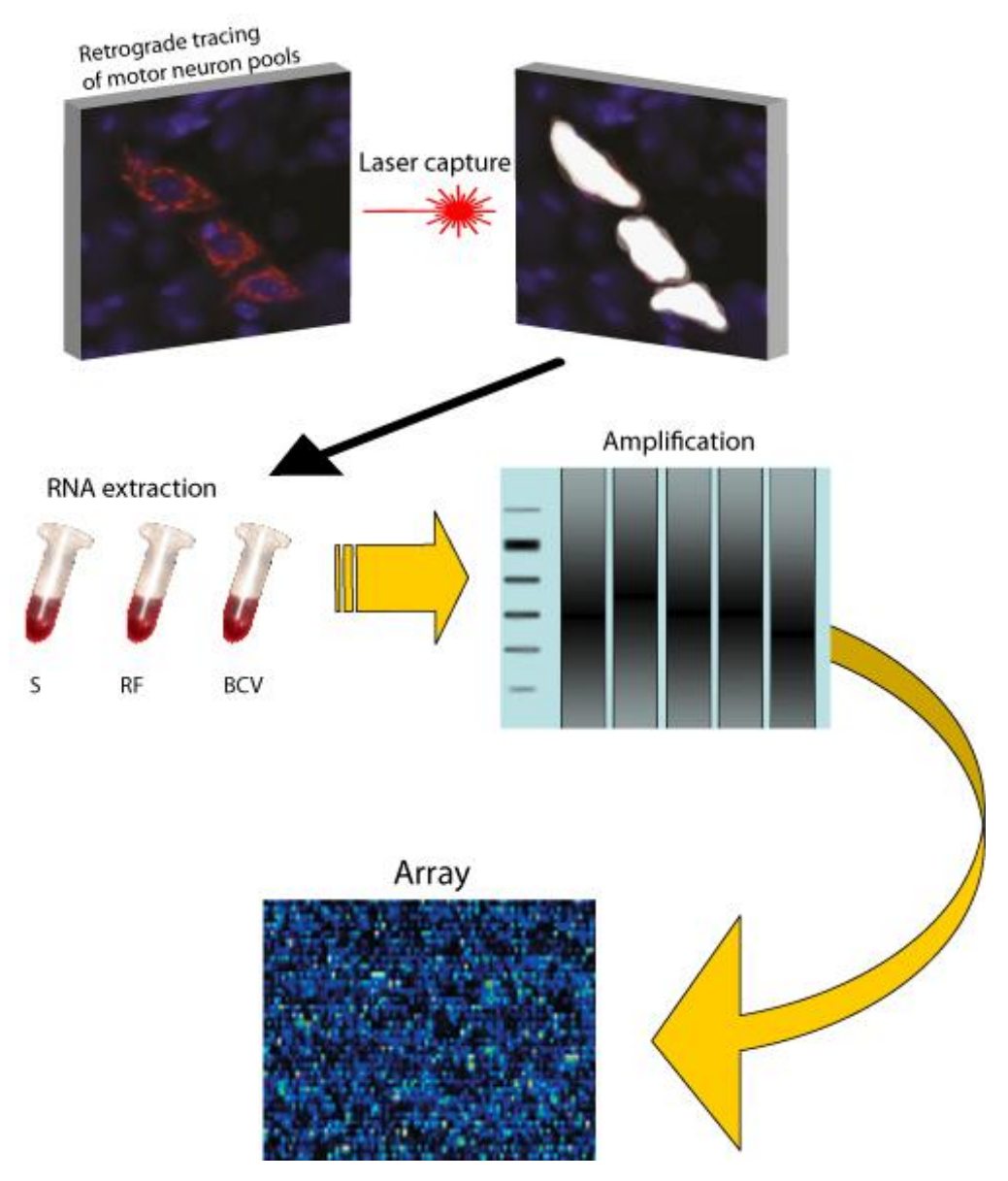

Fig. 9 Flowchart of differential expression analysis

(from top to bottom) Traced MNs were captured by laser capture micro dissection. RNA was extracted from obtained tissue, amplified and labelled for further processing. 10 $\mu \mathrm{g}$ of labelled cDNA were used for detection of differential expression on a Mouse Gene 1.0ST micro array.

For the extraction of mRNA from specific motor pools, flash frozen spinal cords from injected animals were embedded into OCT and cut into $10 \mu \mathrm{m}$ sections and fluorescence dependent laser directed micro capture was performed (Fig. 9). Using this method we were able to obtain mRNA in the ng concentration range, from about 40 labelled cells. For each MN subtype, RNA was extracted from 4 animals and separately processed on an Affymetrix whole transcriptome array (Fig. 9).

The micro array analysis of three MN pools showed a clear pattern in differential expression (Fig. 10). Interestingly, one can clearly distinguish distinct group of genes that are specifically up or down regulated between the MN subtypes. The genes 
expressed in $B C V$ and $S M N$ s have overall quite similar expression levels, whereas, the $R F$ MNs expression profile showed an opposite pattern, with a minor exception of a small set of genes. Herein, $R F$ and $S$ differ from the $B C V$ profile, indicating some $B C V$-specific gene regulation (Fig. 10).

\section{Gene expression profile of motor neuron subtypes}

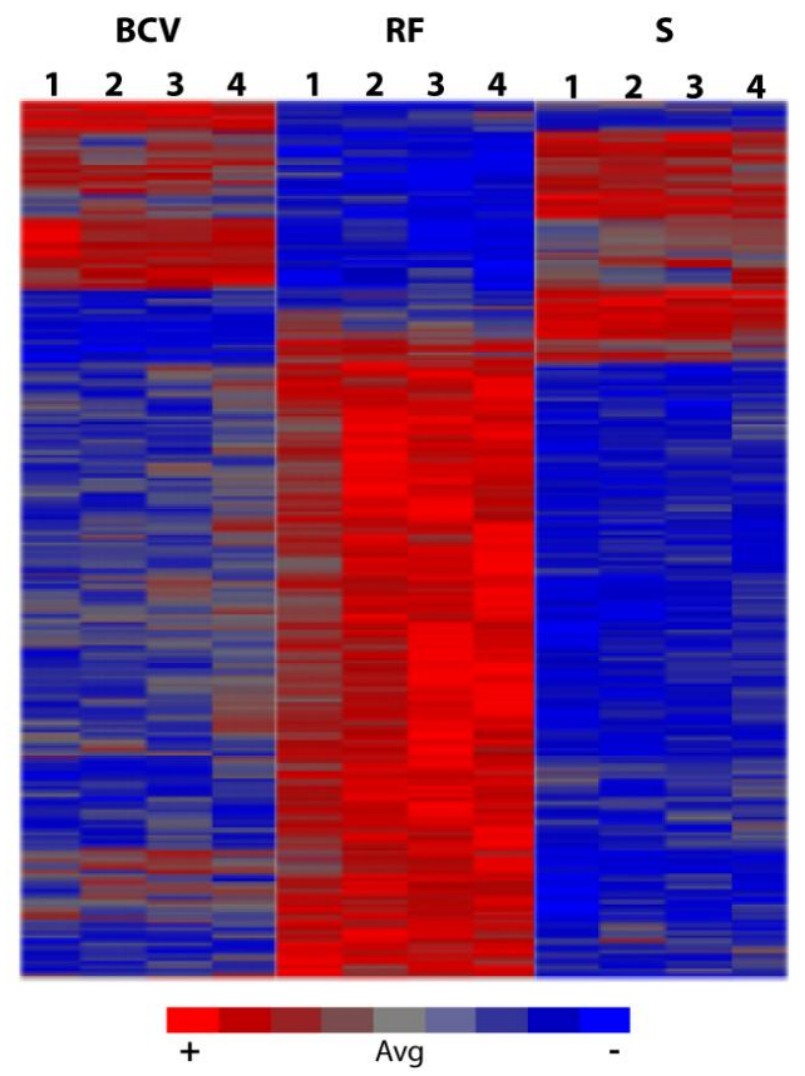

Fig. 10 Array of gene expression profiling

$B C V$ (resistant) compared to $R F$ (vulnerable) and $S$ (relatively resistant) MNs, each with $\mathrm{n}=4$. Heatmap was created from raw data without any filters, showing expression levels relative to each other. 


\subsection{Markers for resistant MNs}

The high number of differentially expressed genes caused problems of structuring the results and filter out possible $\mathrm{MN}$ vulnerability relevant data. Therefore, potential functional networks of differential expressed genes were analysed utilizing the pathway analysis tool IPA (Ingenuity Systems). The tool compared overall changes in gene expression levels in one subset of MNs relative to the expression in one or both of the other subsets of MNs. False positive candidates were excluded by a p-value cut-off of $p<0.05$ and a relative expression value cut-off of 100 . Genes that did not fit these criteria were not further investigated. Networks that each had a minimum of ten hits per pathway were considered. Comparison of differentially expressed genes in the $B C V$ MNs with the expression levels of these genes in $S$ MNs implicated two distinct networks. These networks include genes involved in genetic disorders, nutritional disease, cellular development and carbohydrate metabolism, molecular transport and small molecule biochemistry (Fig. 11). Interestingly, genes of these pathways with low expression in $S \mathrm{MNs}$, also show low expression in the $R F \mathrm{MNs}$. Hence, these networks show genes with a highly specific expression restricted to $B C V$ MNs. Some of the candidate genes retrieved from these analysis have already been shown to play a role in neuronal development and protection, such as brain expressed gene 1 (Bex1) and neurensin 1 (Nrsn1). Interestingly, Calbindin 2 (Calb2) which has an important role in $\mathrm{Ca}^{2+}$ buffering normally has a very low expression level in MNs compared to the other types of neurons. Calb2 shows a five-fold higher expression in BCV MNs when compared to RFMNs (Fig.11). 


\section{Genetic disorder, nutritional disease, cellular development}

\begin{tabular}{|c|c|c|c|c|c|c|c|}
\hline & & & & fold ove & pres & Vsignific & \\
\hline & BCV & $\mathrm{RF}$ & S & $\mathrm{BCV} / \mathrm{RF}$ & & BCVIS & \\
\hline $\begin{array}{l}\text { Bex1 } \\
\text { Cacna2d }\end{array}$ & & & & $\begin{array}{l}3,4 \\
44\end{array}$ & : & $\begin{array}{l}4,9 \\
22\end{array}$ & : \\
\hline $\begin{array}{l}\text { Cachaza } \\
\text { Calb2 }\end{array}$ & & & & & . & $\begin{array}{l}2,2 \\
2,4\end{array}$ & . \\
\hline Clic1 & & & & 2,6 & . & 3,3 & $*$ \\
\hline Comt1 & & & & 2,3 & . & 2,2 & . \\
\hline Ctss & & & & 1,7 & . & 2,3 & * \\
\hline Dgkk & & & & 1,8 & n.s. & 2,3 & . \\
\hline Ecel1 & & & & 3,0 & $\cdots$ & 2,2 & $\ldots$ \\
\hline Fads3 & & & & 1,4 & n.s. & 2,1 & * \\
\hline Fscn1 & & & & 1,6 & $\cdot$ & 2,0 & . \\
\hline Ifi30 & & & & 1,5 & n.s. & 2,2 & * \\
\hline Ly6a & & & & 1,2 & n.s. & 2,3 & . \\
\hline Pip2 & & & & 2,1 & $\cdot$ & 2,6 & * \\
\hline Scn9a & & & & 2,4 & n.s. & 11,5 & * \\
\hline Srd5a2 & & & & 20,4 & $*$ & 25,3 & .. \\
\hline
\end{tabular}

Carbohydrate metabolism, molecular transport, small molecule biochemistry

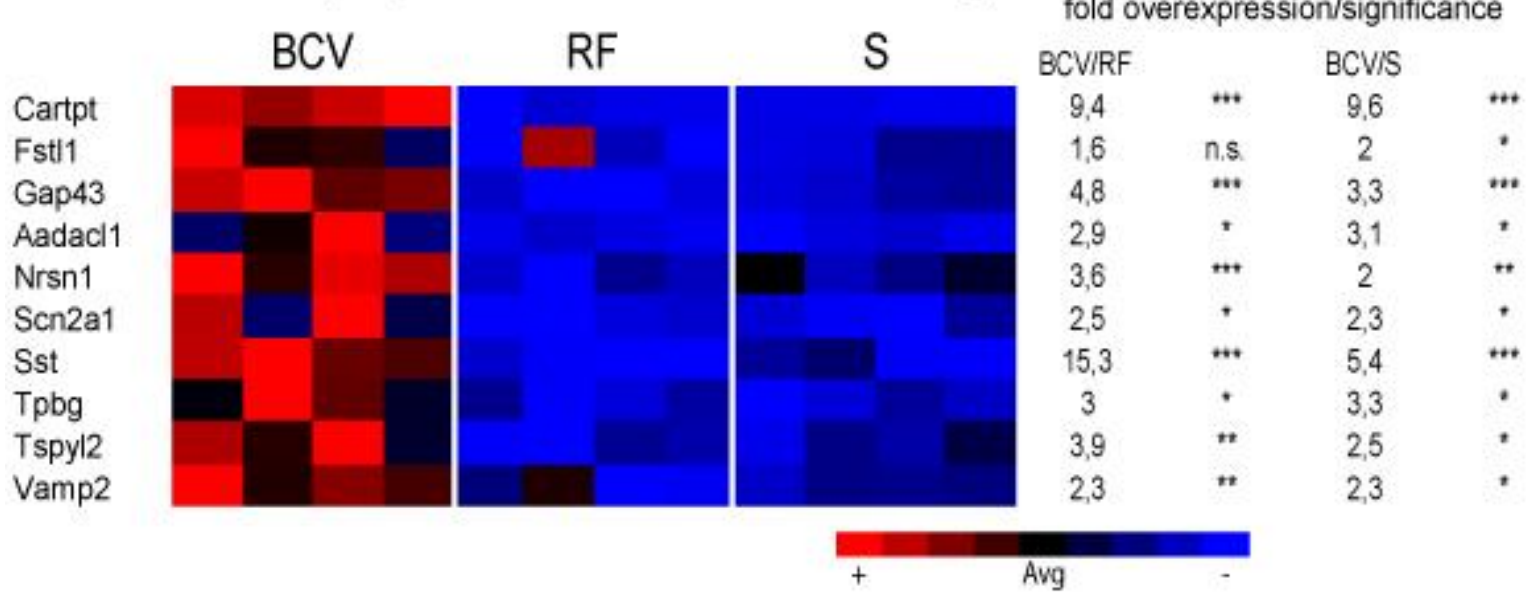

Fig. 11 Heatmaps of functional network analysis in BCV MNs

Networks specific for differentially expressed genes in the BCV (resistant) MNs compared with the $S$ MNs. Threshold of relative expression levels was prior set to 100 to avoid false positives. Fold overexpression and significance were calculated in respect to expression levels in $S$ and $R F$ MNs $(n=4)$. Significant differences are indicated (t-student's test) ${ }^{*} p<0.05,{ }^{* *} p<0.01,{ }^{* * *} p<0.001$ 

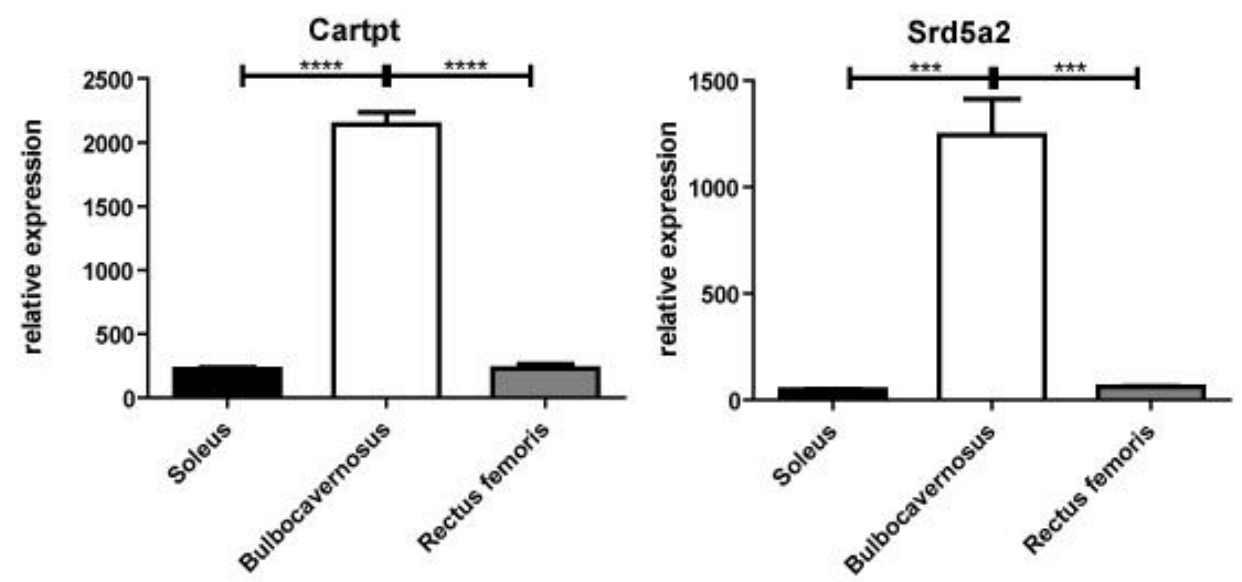

Fig. 12 Relative expression levels of Cartpt and Srd5a2.

Array data showing the relative expression levels of Cartpt and Srd5a2 in BCV MNs compared to their expression in $S$ and $R F M N s(n=4)$. Significant differences are indicated (t-student's test) * $p<0.05$, ** $p<0.01,{ }^{* * *} p<0.005,{ }^{* * * *} p<0.001$
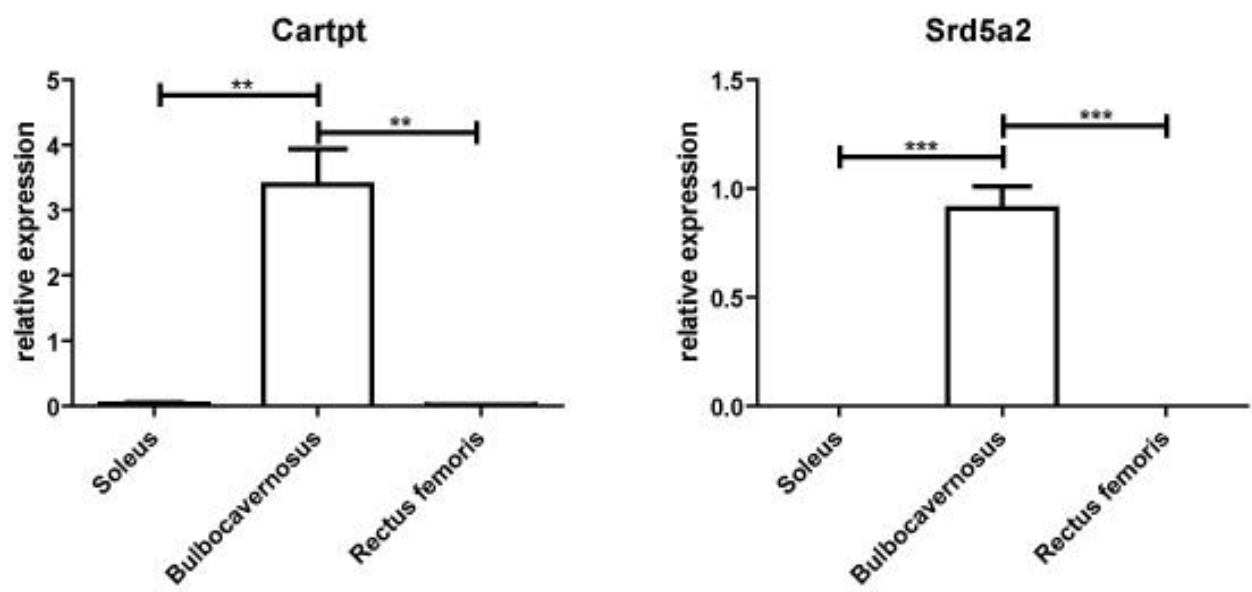

Fig. 13 qPCR of Cartpt and Srd5a2

Relative expression levels of Cartpt and Srd5a2 after qPCR in BCV MNs compared with their expression in $S$ and $R F$ MNs $(\mathrm{n}=4)$. Expression of alpha tubulin $1 B$ was set as 1 , to which the relative expression levels were normalized. Significant differences are indicated (t-student's test) * $p<0.05$, * $p<0.01,{ }^{* * *} p<0.005,{ }^{* * * *} p<0.001$

Among the genes that showed the highest differential expression were Cocaine and amphetamine regulated transcript prepropeptide (Cartpt/Cart) and steroid 5 alphareductase 2 (Srd5a2) (Fig. 12). Considering a very strong difference in expression, 
these genes were thought to serve as suitable candidates for markers of the resistant MN subtype. The array data of these two genes was confirmed with qPCR (Fig. 13), wherein expression of Cartpt and Srd5a2 was shown to be exclusively in BCV MNs. In order to use these genes as markers for a subset of MNs, confirmation via in situ hybridization was necessary. The expression of Cartpt is clearly confined to the central canal area in lumbar to sacral spinal cord of P20 mice (Fig. 14), which corresponds to the localization of $B C V M N s$ in the medially lower lumbar region of the spinal cord (Fig. 8).

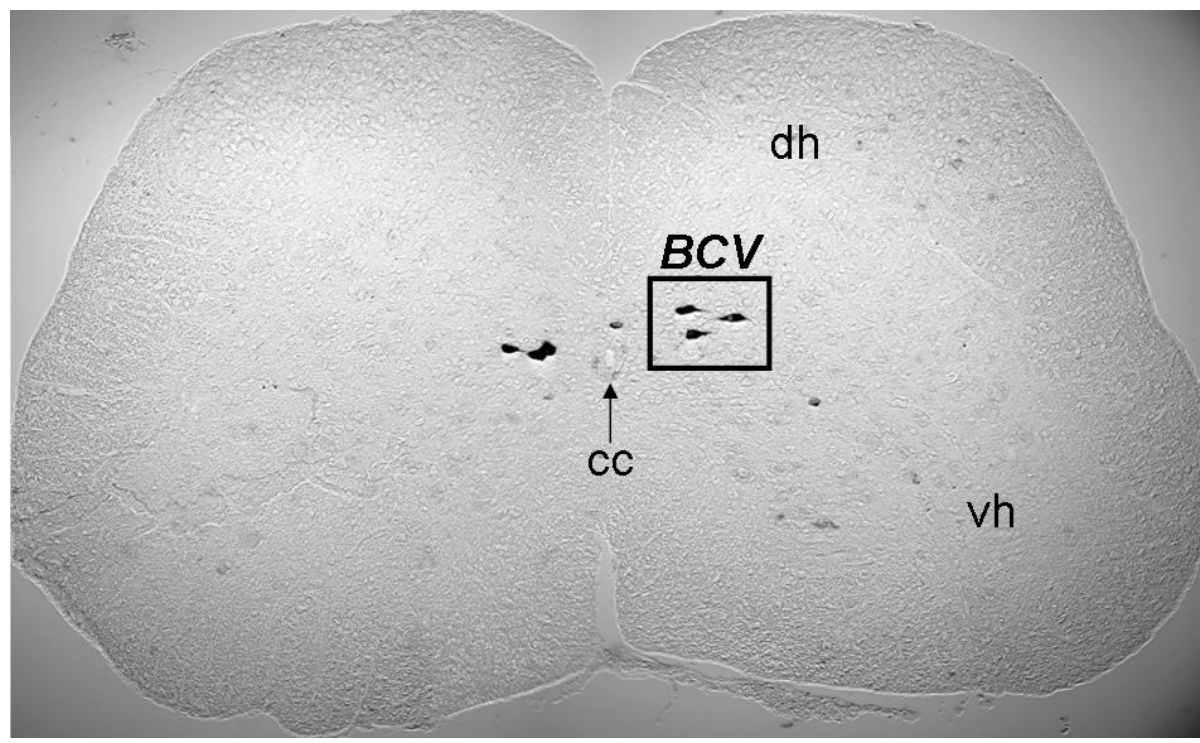

\section{Fig. 14 In situ analysis of Cartpt}

The picture shows a staining of Cartpt after in situ hybridization on $14 \mu \mathrm{m}$ P20 mouse spinal cord sections. Close restriction of stained MNs to the central canal, which has shown to be the area of $B C V$ MNs. $\mathrm{dh}=$ dorsal horn, $\mathrm{vh}=$ ventral horn, $\mathrm{cc}=$ central canal

Taken together, these results confirm restricted expression of Cartpt to BCV MNs, but not to other MNs, and thereby can be considered as a novel marker for resistant MNs. 


\subsection{Functional network analysis of $S$ candidates}

The network analysis of $S \mathrm{MN}$ vs. BCV MN expression levels revealed three networks restricted to differentially expressed genes in $S$ MNs. Whereas two functional networks were disease related and contained genes involved in neurological diseases, cancer, genetic disorders and psychological diseases, the third network though, displayed cell cycle functions, such as RNA post-transcriptional modification, lipid metabolism and nucleic acid modification (Fig. 15).

\section{Neurological disease, behavior psycological disorders}

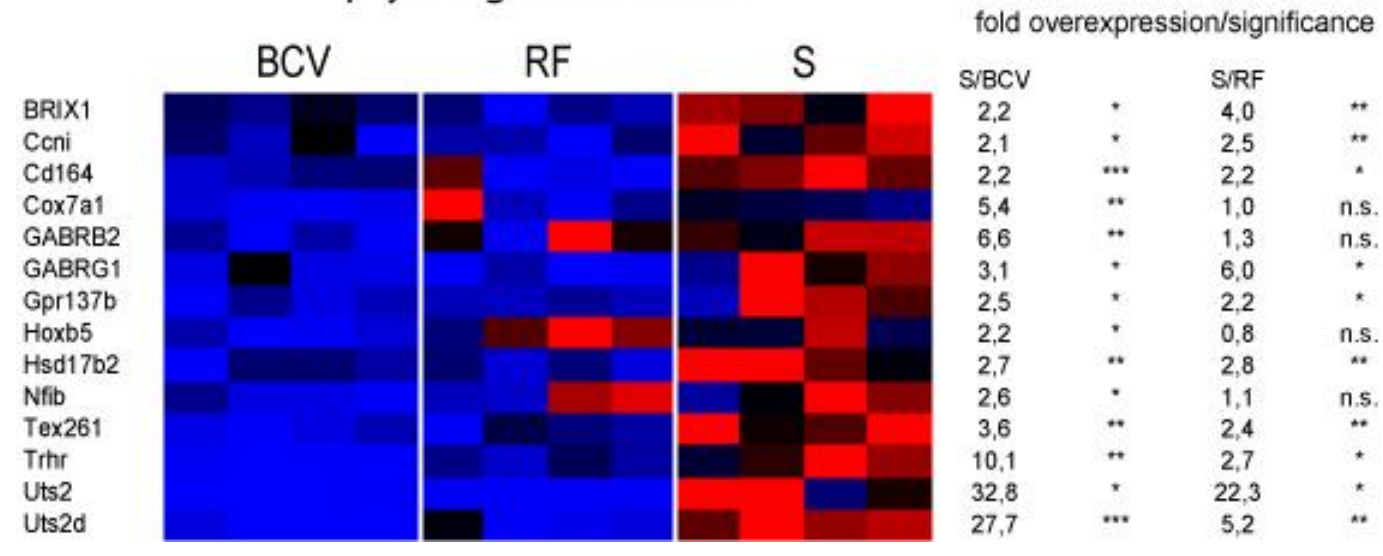

Cancer, genetic disorder, neurological disease

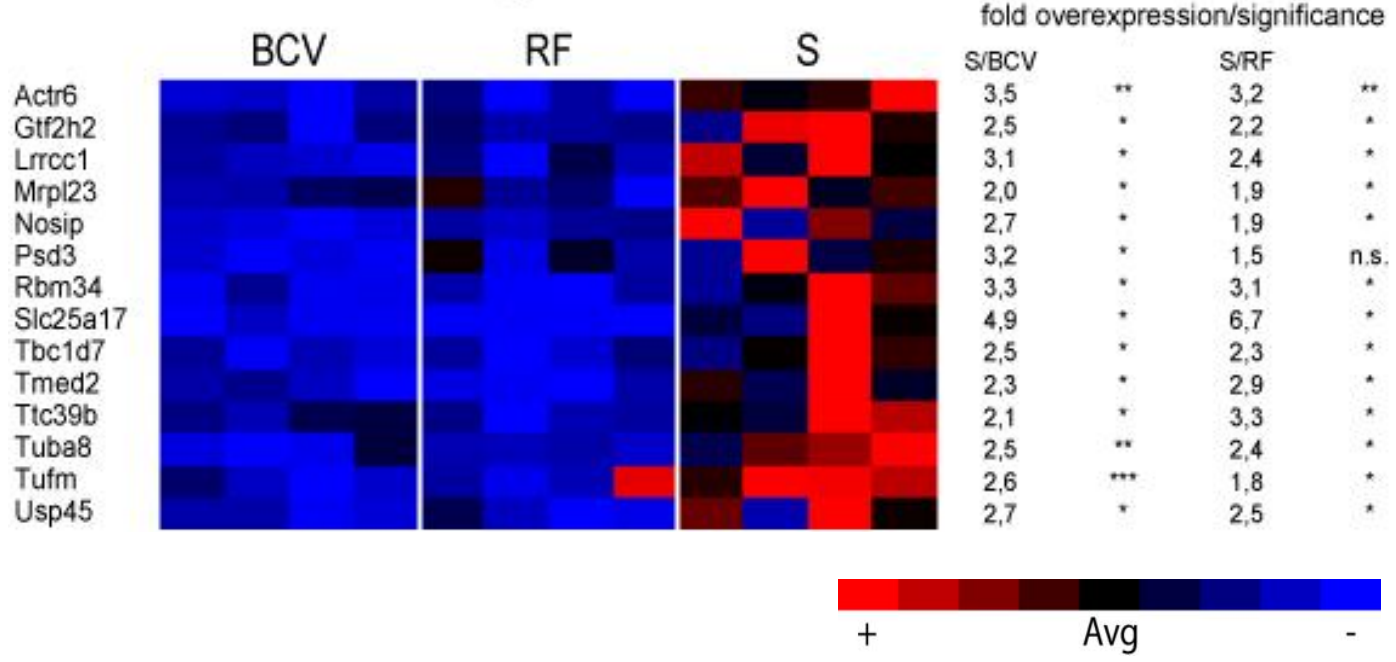

Fig. 15 Heatmaps of disease related S MN candidates

Networks of candidates implicated in disease, specifically differentially expressed genes in $S$ (relatively resistant) MNs compared with the $B C V$ (resistant) MNs. Threshold of relative expression 
levels was prior set to 100 to avoid false positives. Fold overexpression and significance were calculated in respect to expression levels in $B C V$ and $R F$ MNs $(n=4)$. Significant differences are indicated (t-student's test) * $p<0.05,{ }^{* *} p<0.01,{ }^{* * *} p<0.001$
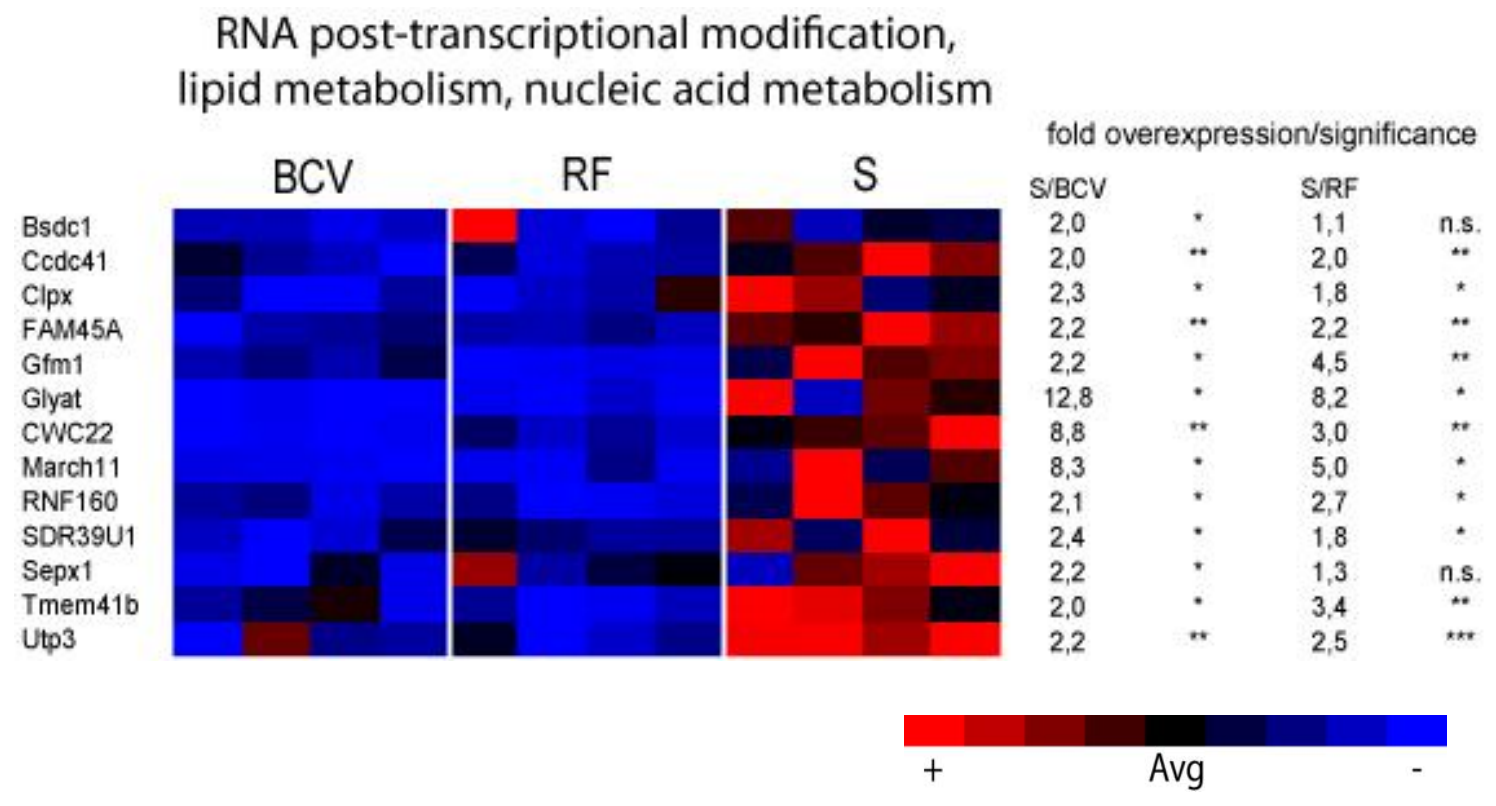

Fig. 16 Heatmap of cell cycle networks in $S$ MNs

Differentially expressed genes in the $S$ (relatively resistant) MNs compared with the $B C V$ MNs. Threshold of relative expression levels was prior set to 100 to avoid false positives. Fold overexpression and significance were calculated in respect to expression levels in $B C V$ and $R F M N s$ $(n=4)$. Significant differences are indicated (t-student's test) ${ }^{*} p<0.05,{ }^{* *} p<0.01,{ }^{* * *} p<0.001$

Similar to analysis of the $B C V$-specific genes, candidates that are genetic markers for the $S$ MNs were found. Relative expression levels of urotensin 2 (Uts2) and urotensin 2 domain containing (Uts2d) showed an extremely high differential expression in $S$ MNs (Fig. 17). Since Utd2d showed a minor expression difference towards $R F \mathrm{MNs}$, we decided to further investigate only Uts2. Verification of Uts2 via qPCR revealed highly significant differential expression particularly in $S$ MNs (Fig. 18). Additionally, an in situ probe was successful designed for Uts2. Thus, for Uts2 could be confirmed that expression was limited to $S$ MNs via qPCR and in situ hybridization and thereby gained as marker for relatively resistant MNs (Fig. 18, 19). 

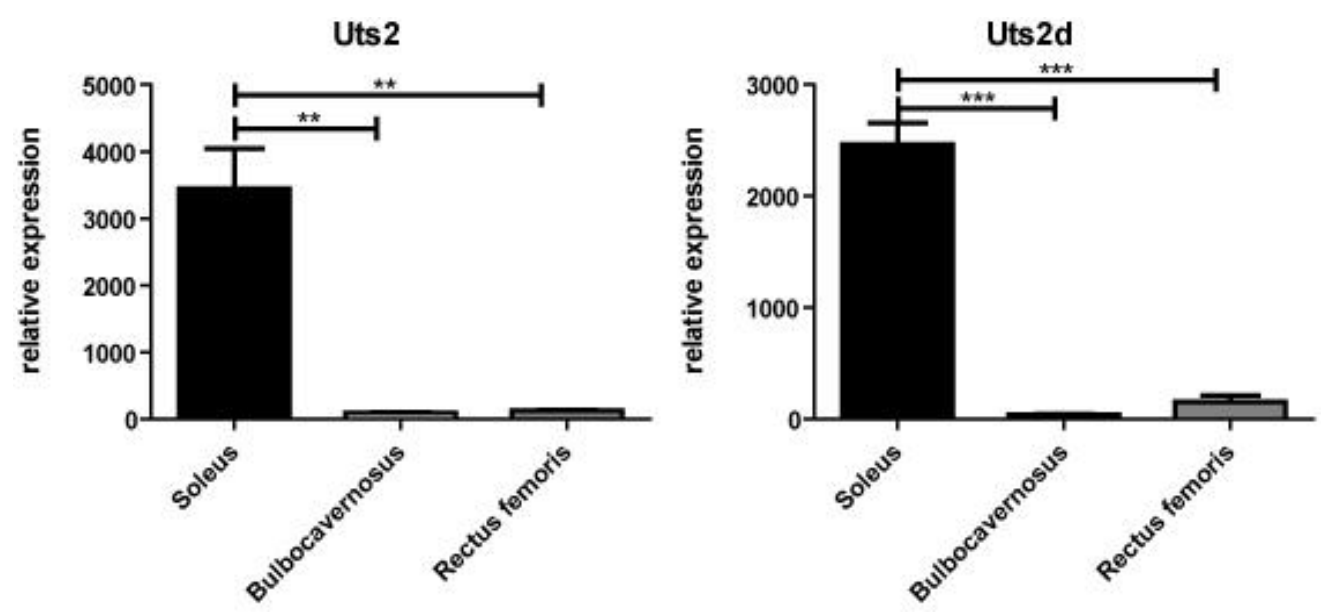

Fig. 17 Relative expression levels of Uts2 and Uts2d

Array data showing the relative expression levels of Uts2 and Uts2d in $S$ MNs compared to their expression in $B C V$ and $R F$ MNs $(\mathrm{n}=4)$. Significant differences are indicated (t-student's test) ${ }^{*} \mathrm{p}<$ $0.05,{ }^{* *} p<0.01,{ }^{* * *} p<0.005,{ }^{* * * *} p<0.001$

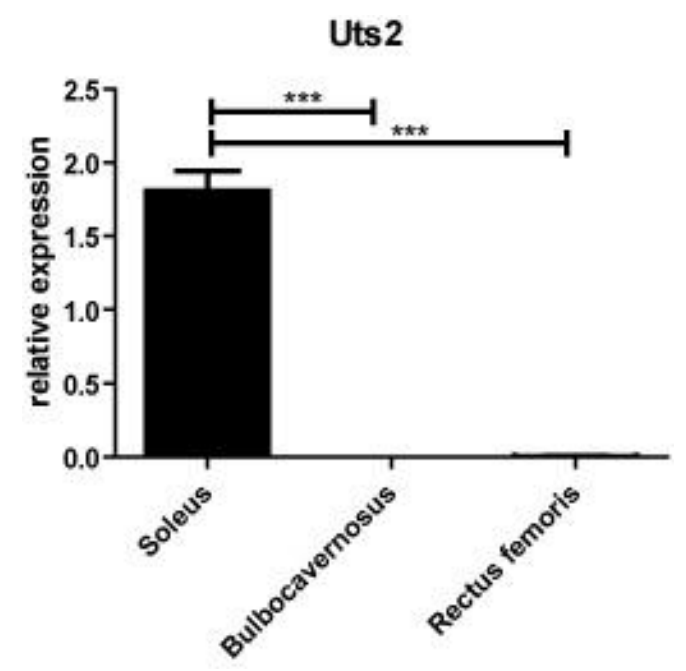

\section{Fig. 18 qPCR of Uts2}

Relative expression level of Uts2 in qPCR in $S$ MNs compared with expression in BCV and RF MNs $(n=4)$. Expression of alpha tubulin $1 B$ was set as 1 , to which the relative expression levels were normalized. Significant differences are indicated (t-student's test) ${ }^{*} p<0.05,{ }^{* *} p<0.01,{ }^{* * *} p<0.005$, **** $p<0.001$

In situ hybridization of Uts2 successfully validated array and qPCR findings. The localization of relatively resistant $S$ MNs in mouse spinal cord could be found within the dorsal part of the ventral horn (Fig. 19A). 

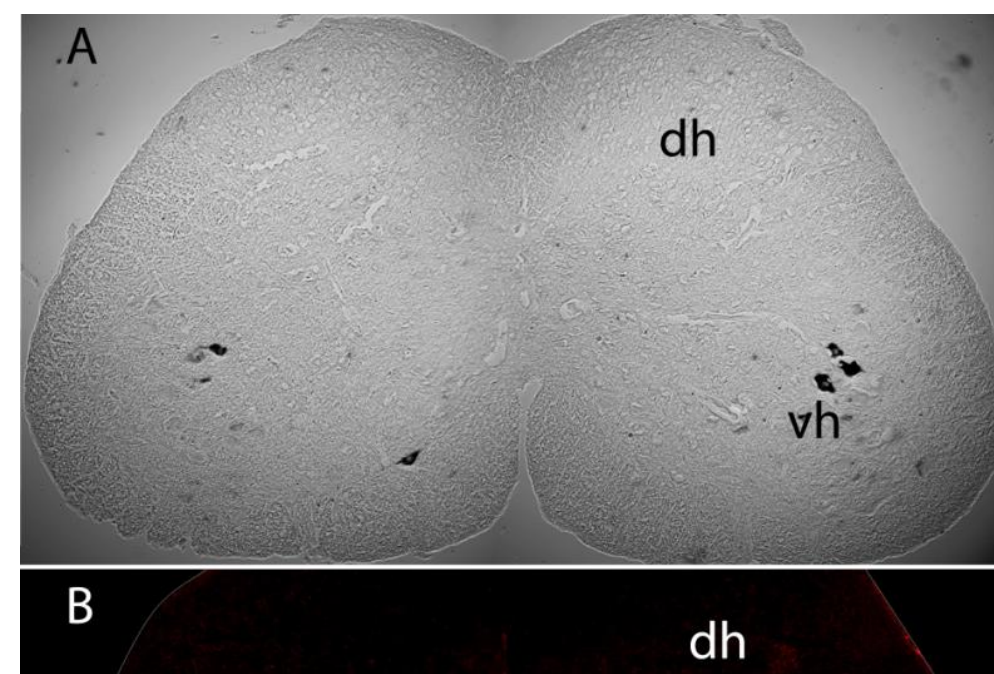

\section{$\mathrm{vh}$}

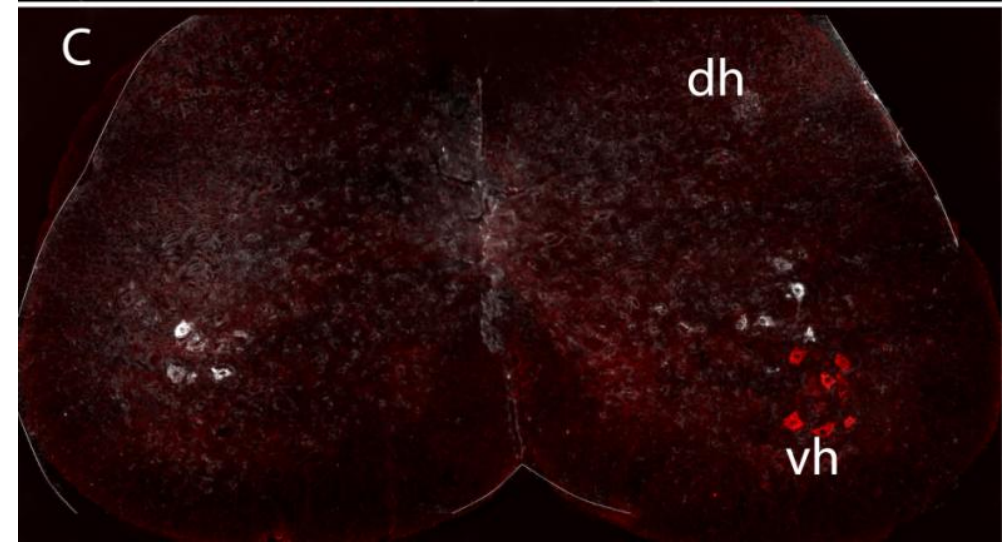

Fig. 19 In situ analysis of Uts2

(A) Staining of Uts2 after in situ hybridization, showing close restriction of stained MNs to the dorsal part of the ventral horn. (B) Shows a retrograde tracing of $S$ MNs. Choleratoxin subunit B (CTXB) in red, labelled the MNs innervating the $S$ muscle, showing the localization of $S$ MNs in the dorsal part of the ventral horn. Lines indicate the shape of the spinal cord cross section. (C) is an overlay of retrograde traced $\mathrm{MNs}$ innervating the $R F$ (red) and the $S$ (gray) muscle. All sections were obtained from similar regions in lumbar spinal cord. Pictures were taken from $14 \mu \mathrm{m}$ cryo sections of P20 mouse spinal cord.

Bilateral injection into the $S$ muscle gave clear localization sites within the spinal cord for the $S$ MNs (Fig. 19B). While relatively resistant (slow) MNs are thought to be 
distributed in a 'salt-and-pepper' fashion, it is expected that most of the MNs innervating the $\mathrm{m}$. Soleus muscle show expression of a putative slow MN marker gene. These sites overlap with the localization for the relatively resistant MNs that were obtained from the in situ hybridization. The enrichment of Uts2 expression in $S$ MNs is therefore consistent with a selective localization of Uts2 in resistant slow MNs. The retrograde traced MNs of the RF showed a different localization in the ventral horn of mouse spinal cord (Fig. 19C).

Since the expression profiles of $B C V$ and $S$ MNs showed high similarities, it was assumed that pathways conferring MN resistance would be - at least in part - shared by the two resistant MN types, the $B C V$ and the $S$ MNs, but not by the RF MNs. Seven networks were found, which were organized into three groups. The first group contained disease relevant networks (Fig. 20). Interestingly, several of these genes are involved in mitochondrial regulation and metabolism: translocase of outer mitochondrial membrane 70 homolog A (Tomm70a), which is involved in the import of proteins into mitochondria, voltage-dependent anion channel 2 (Vdac2), a voltage gated ion channel responsible for transport of metabolites and mitochondria-induced apoptosis, aldolase $\mathrm{C}(\mathrm{Aldoc})$, that codes for the enzyme aldolase, NADH dehydrogenase (ubiquinone) Fe-S protein 1 (Ndufs1) and succinate dehydrogenase flavoprotein subunit (Sdha1), which code for the complex I and II subunits of the respiratory chain, respectively.

The second group consisted of cellular functions like assembly, organization and morphology as well as nervous system development and function (Fig. 21), while the third group comprised genes involved in protein synthesis and degradation (Fig. 22). 
Neurological disease,

organismal injury and abnormalities, cell signaling

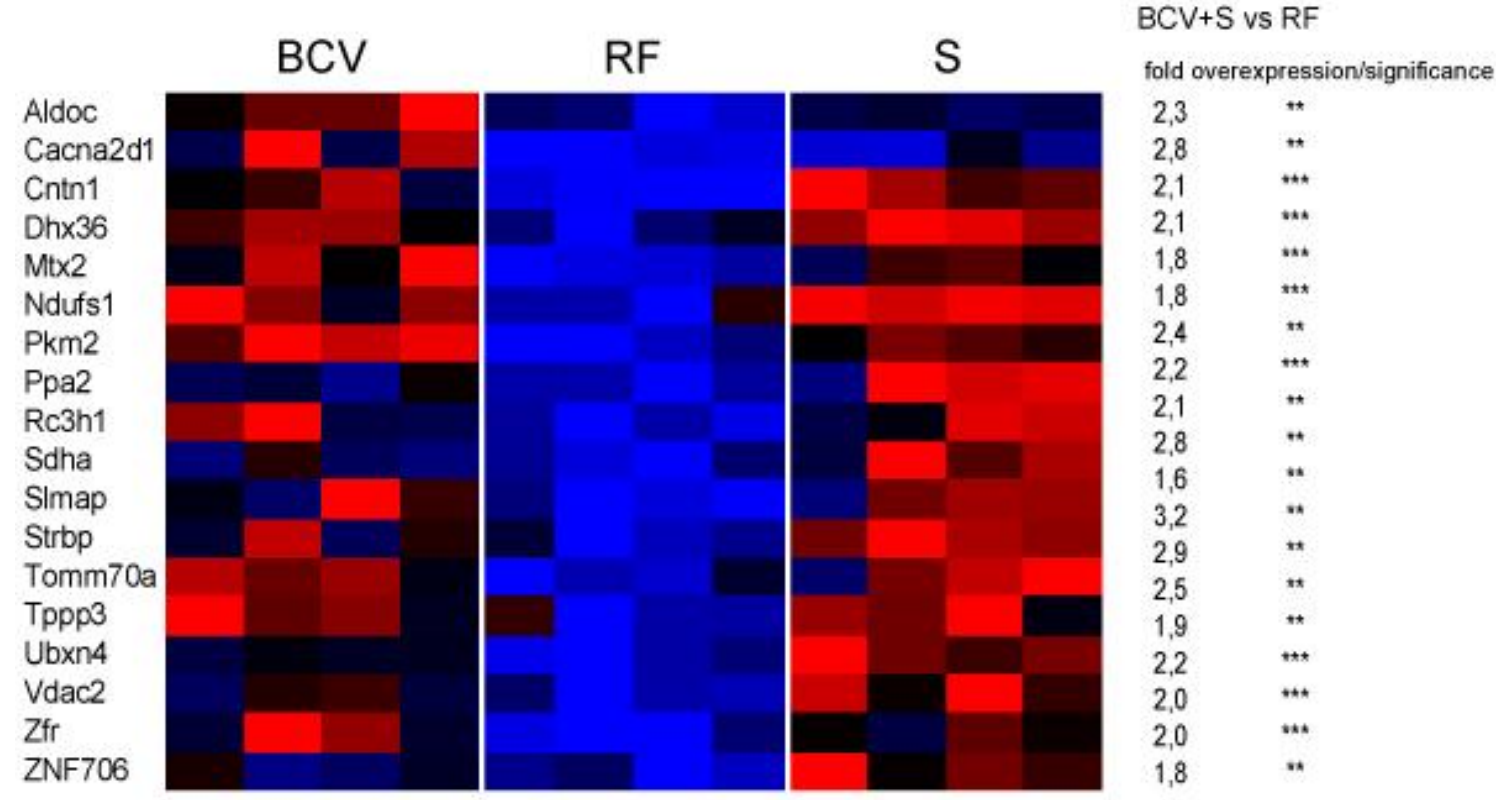

Genetic disorder, metabolic disease, behavior

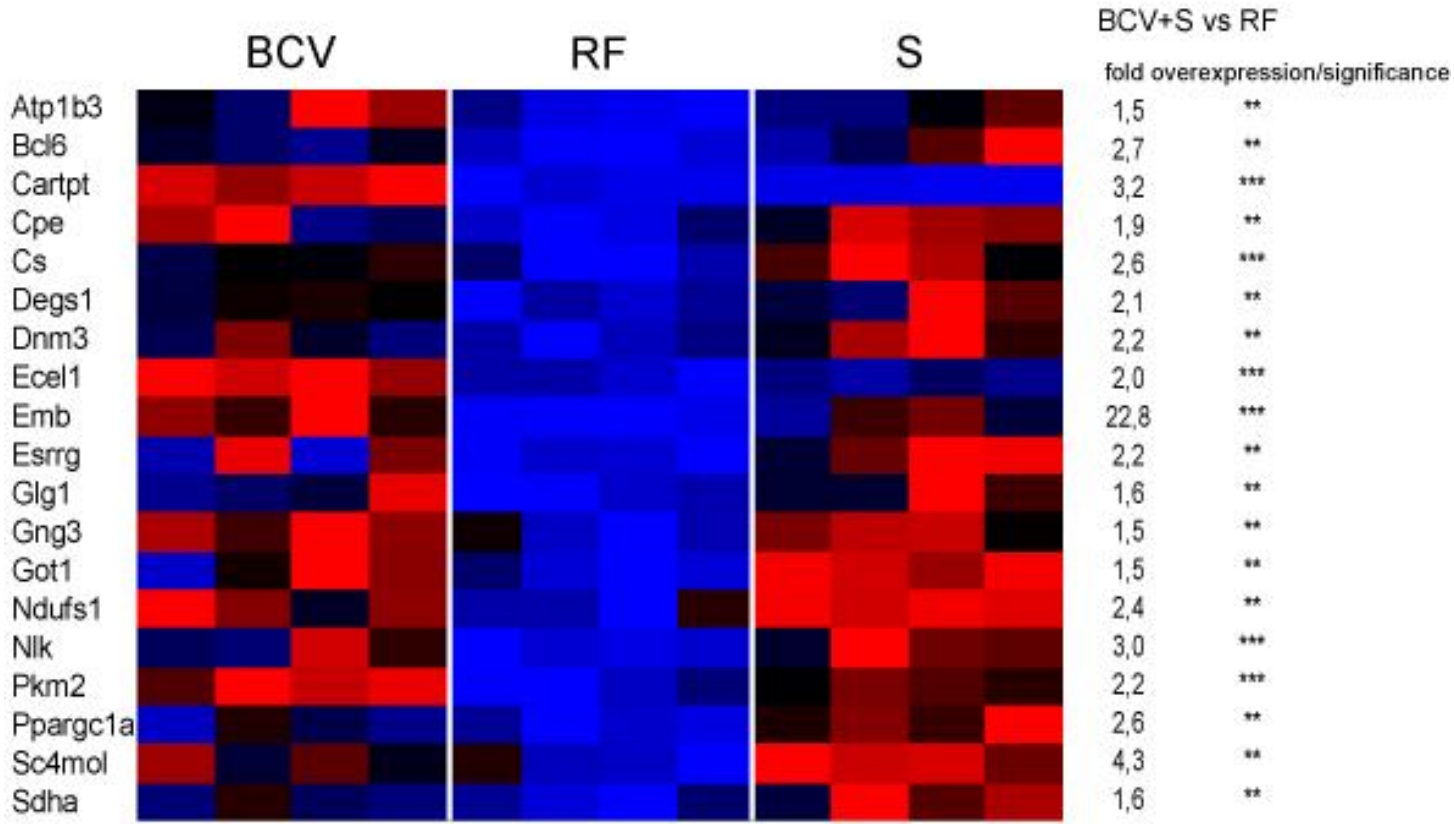


Skeletal and muscular disorders, cell cycle, respiratory system development and function

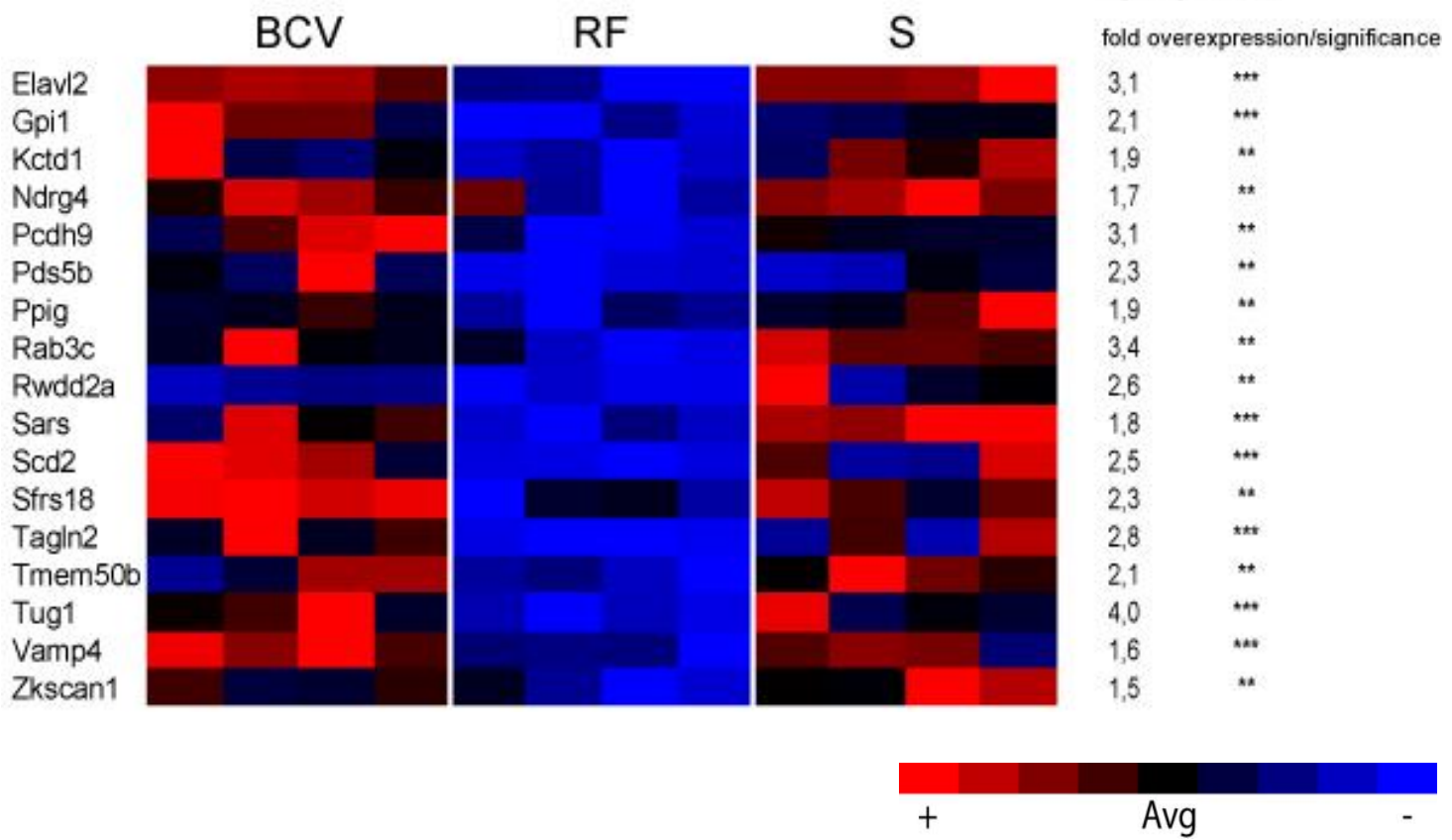

Fig. 20 Heatmaps of disease relevant networks in $B C V$ and $S$ vs. $R F$

Differentially expressed genes in $S$ (relatively resistant) and $B C V$ (resistant) MNs compared to $R F$ MNs. Threshold of relative expression levels was prior set to 100 to avoid false positives. Fold overexpression and significance were calculated in respect to expression levels in RF MNs $(n=4)$. Significant differences are indicated (t-student's test) ${ }^{*} p<0.05,{ }^{* *} p<0.01,{ }^{* * *} p<0.001$

Cellular assembly and organization, cell morphology, nervous system development and function

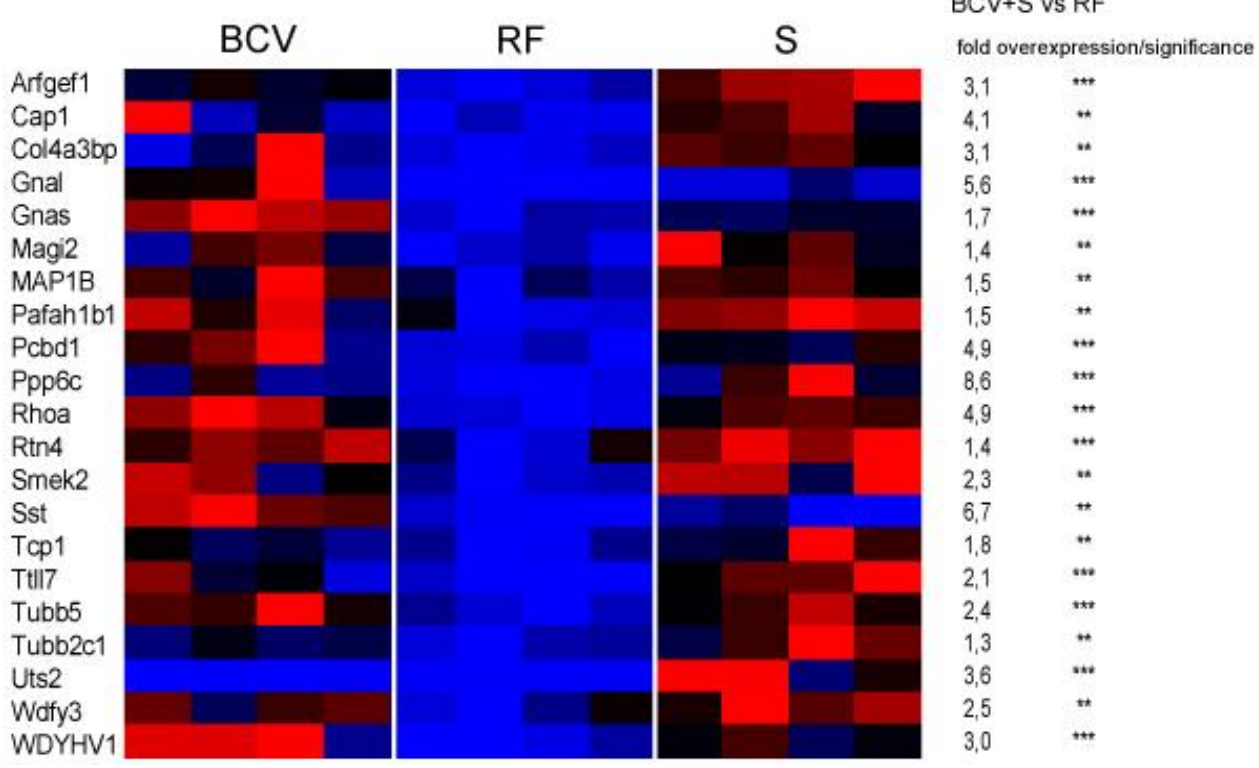


Cellular compromise, cellular assembly and organization, cell-to-cell signaling and interaction

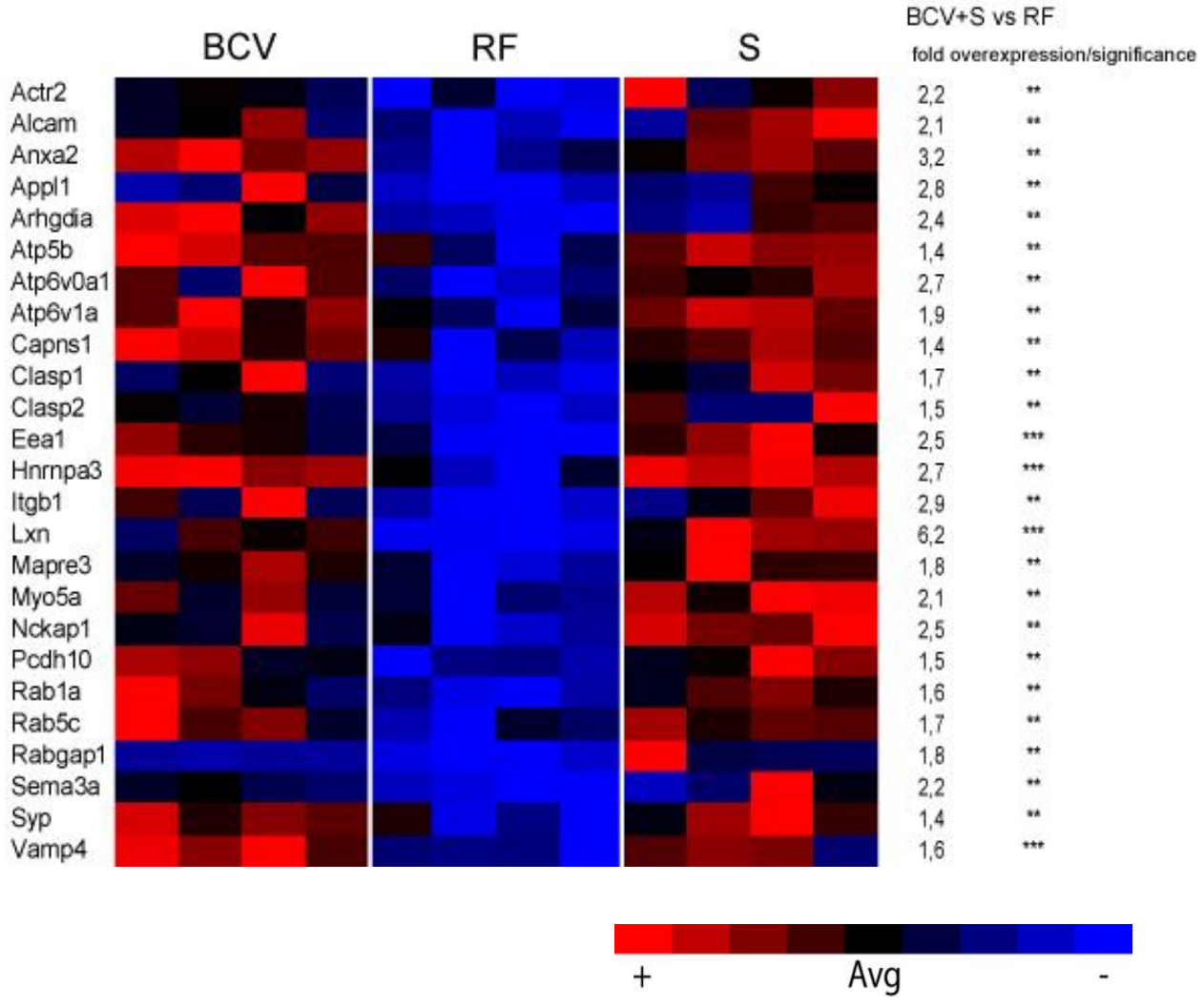

Fig. 21 Heatmaps of cellular functions in $B C V$ and $S$ vs. $R F$

Differentially expressed genes in $S$ (relatively resistant) and $B C V$ (resistant) MNs compared to $R F$ MNs. Threshold of relative expression levels was prior set to 100 to avoid false positives. Fold overexpression and significance were calculated in respect to expression levels in $R F$ MNs $(n=4)$. Significant differences are indicated (t-student's test) ${ }^{*} p<0.05,{ }^{* *} p<0.01,{ }^{* * *} p<0.001$ 


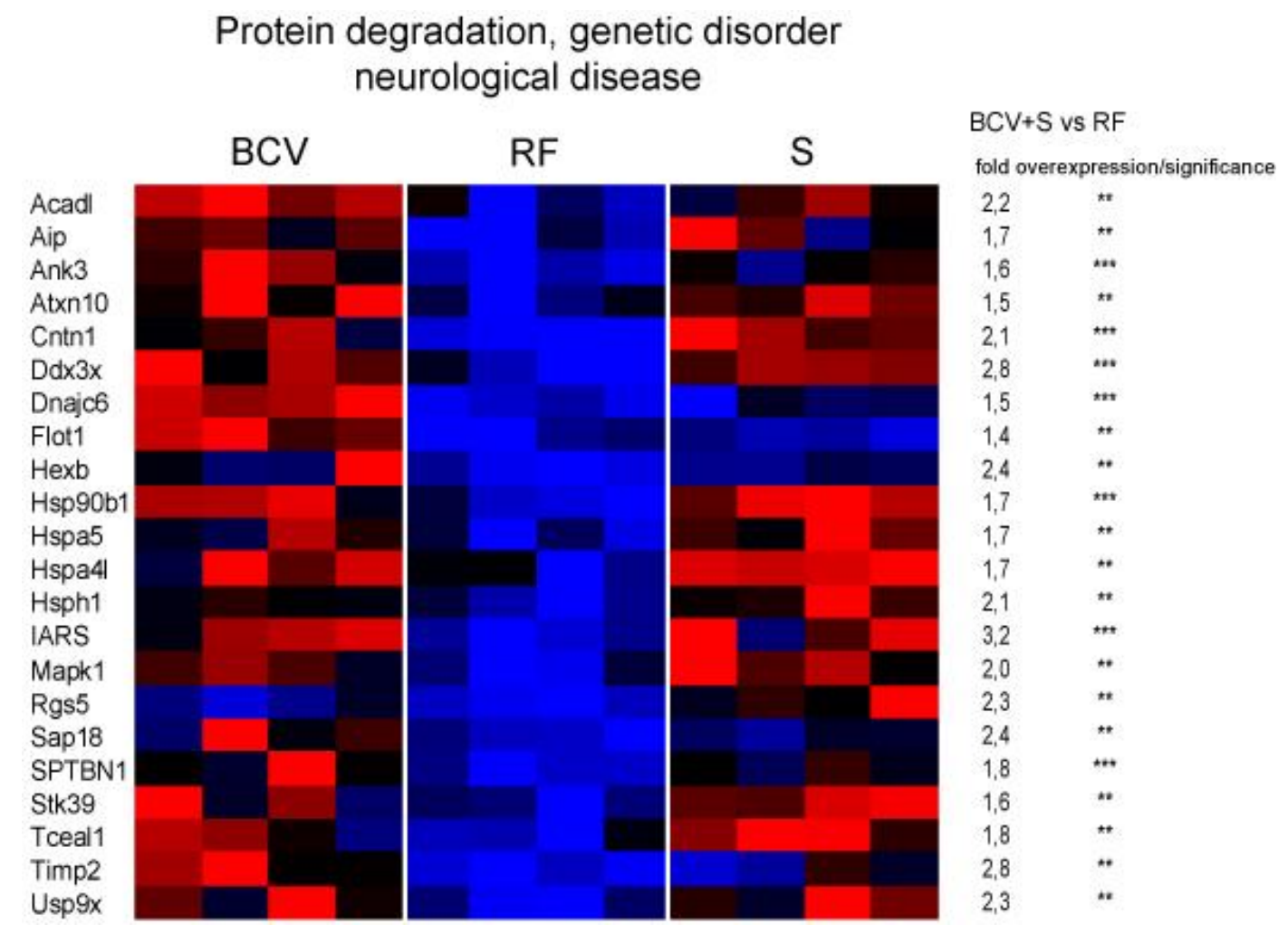

Protein synthesis, cell morphology,

cell to cell signaling and interaction

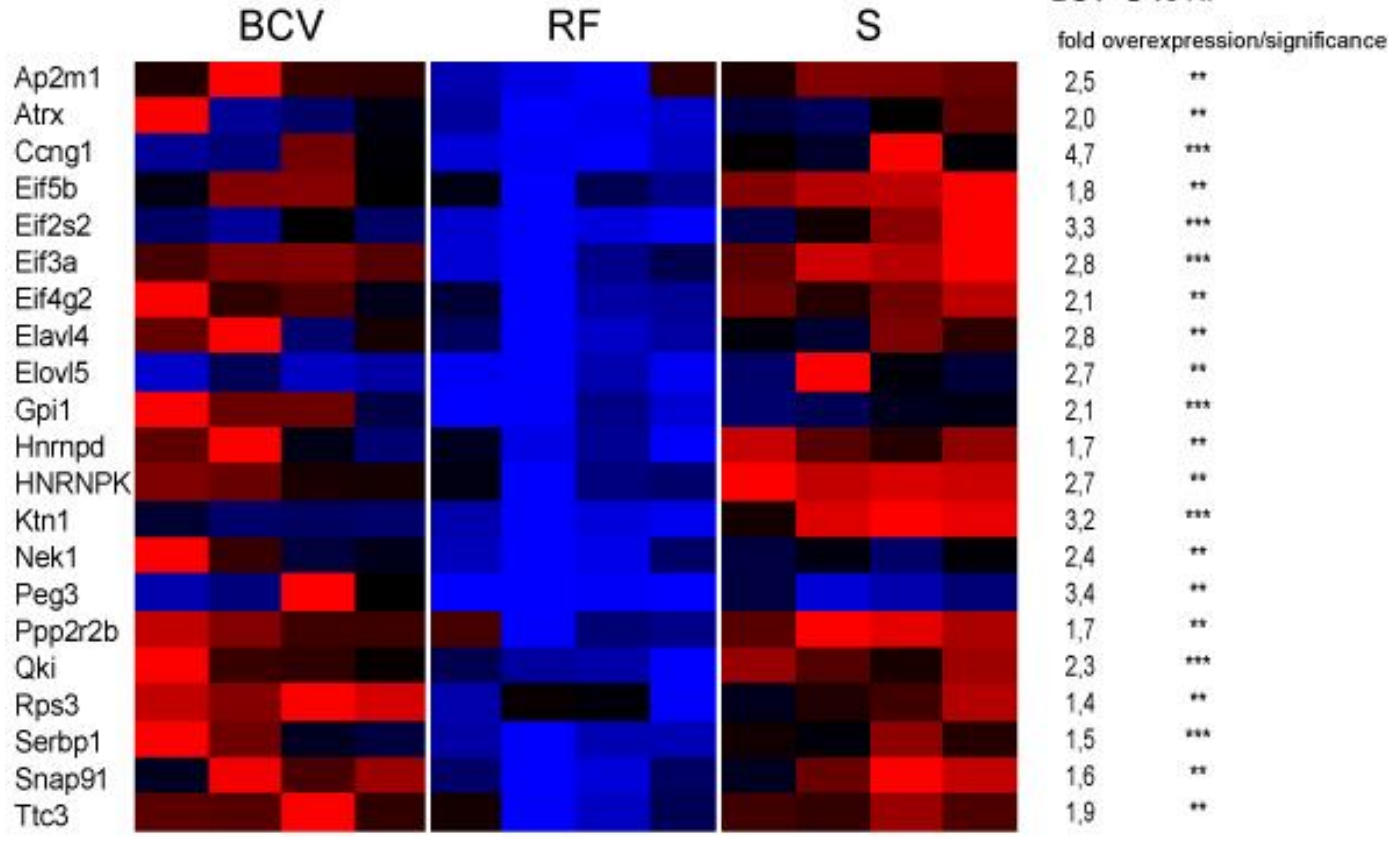

Fig. 22 Heatmaps of protein metabolism in $B C V$ and $S$ vs. $R F$

Differential expressed genes in $S$ (relatively resistant) and $B C V$ (resistant) MNs compared to RF MNs. Threshold of relative expression levels was prior set to 100 to avoid false positives. Fold overexpression and significance were calculated in respect to expression levels in $\operatorname{RF~MNs~}(n=4)$. Significant differences are indicated (t-student's test) ${ }^{*} p<0.05,{ }^{* *} p<0.01,{ }^{* * *} p<0.001$ 
We chose four candidates with high expression levels in $B C V$ and $S$ MNs, but low levels in $R F M N$ s, for further investigation. Embigin $(E m b)$ and latexin $(L x n)$ showed high differential expression compared to $R F \mathrm{MNs}$ and were chosen to serve as markers for $B C V$ and $S$ MNs (Fig. 23). Among those, leukemia inhibitory factor receptor (Lifr) and UBX domain protein 4 (Ubxn4) were of interest as candidates since they have been shown to play a neuroprotective role. The expression of Lifr and $L x n$ in $B C V$ and $S$, but not in RFMNs was confirmed by qPCR (Fig. 24).
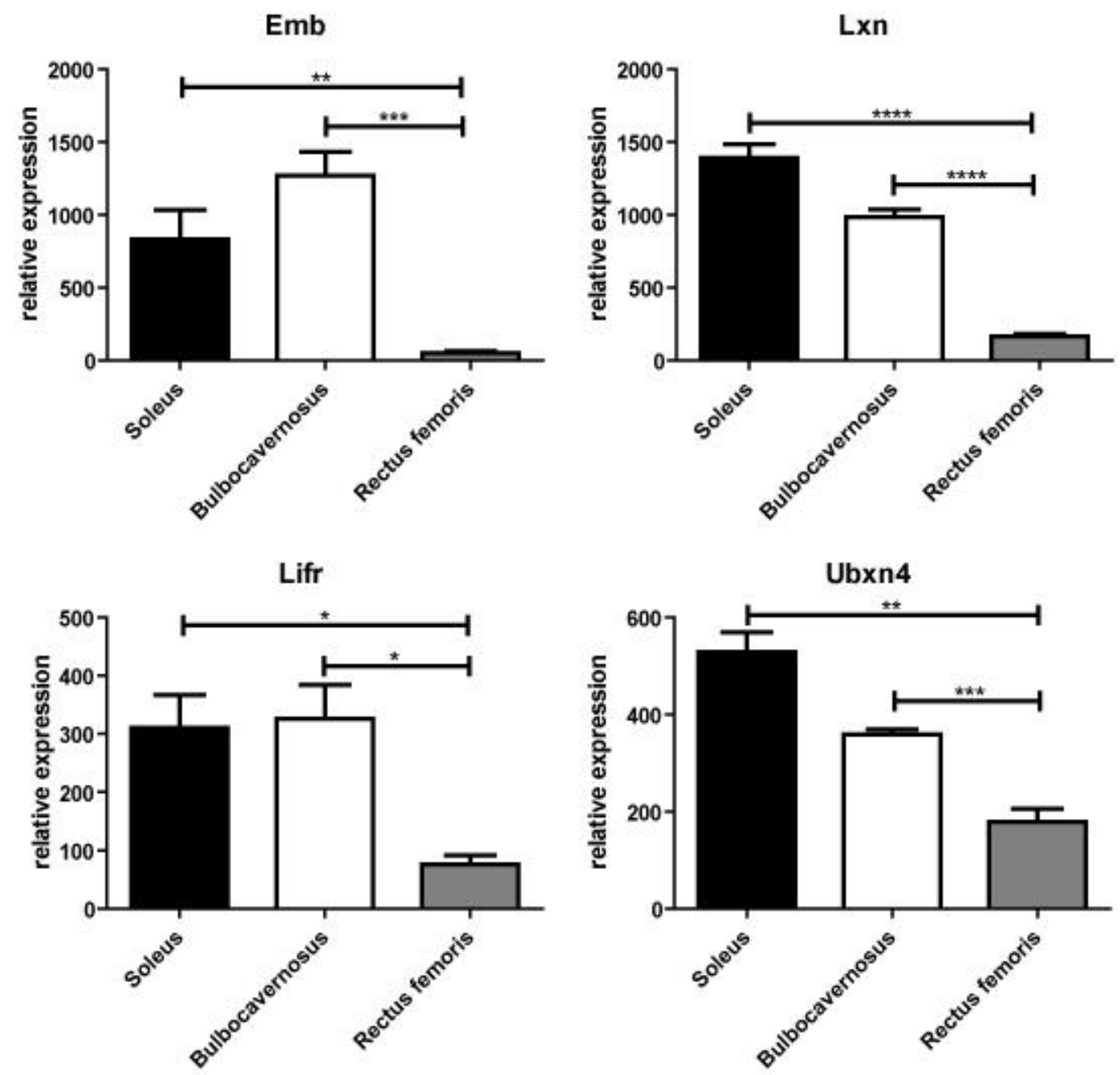

Fig. 23 Relative expression levels of Emb, Lxn, Lifr and Ubxn4

Array data showing the relative expression levels in $B C V$ and $S M N$ s compared to their expression in $R F$ MNs $(n=4)$. Significant differences are indicated (t-student's test) ${ }^{*} p<0.05,{ }^{* *} p<0.01,{ }^{* * *} p<$ $0.005,{ }^{* * * *} \mathrm{p}<0.001$ 

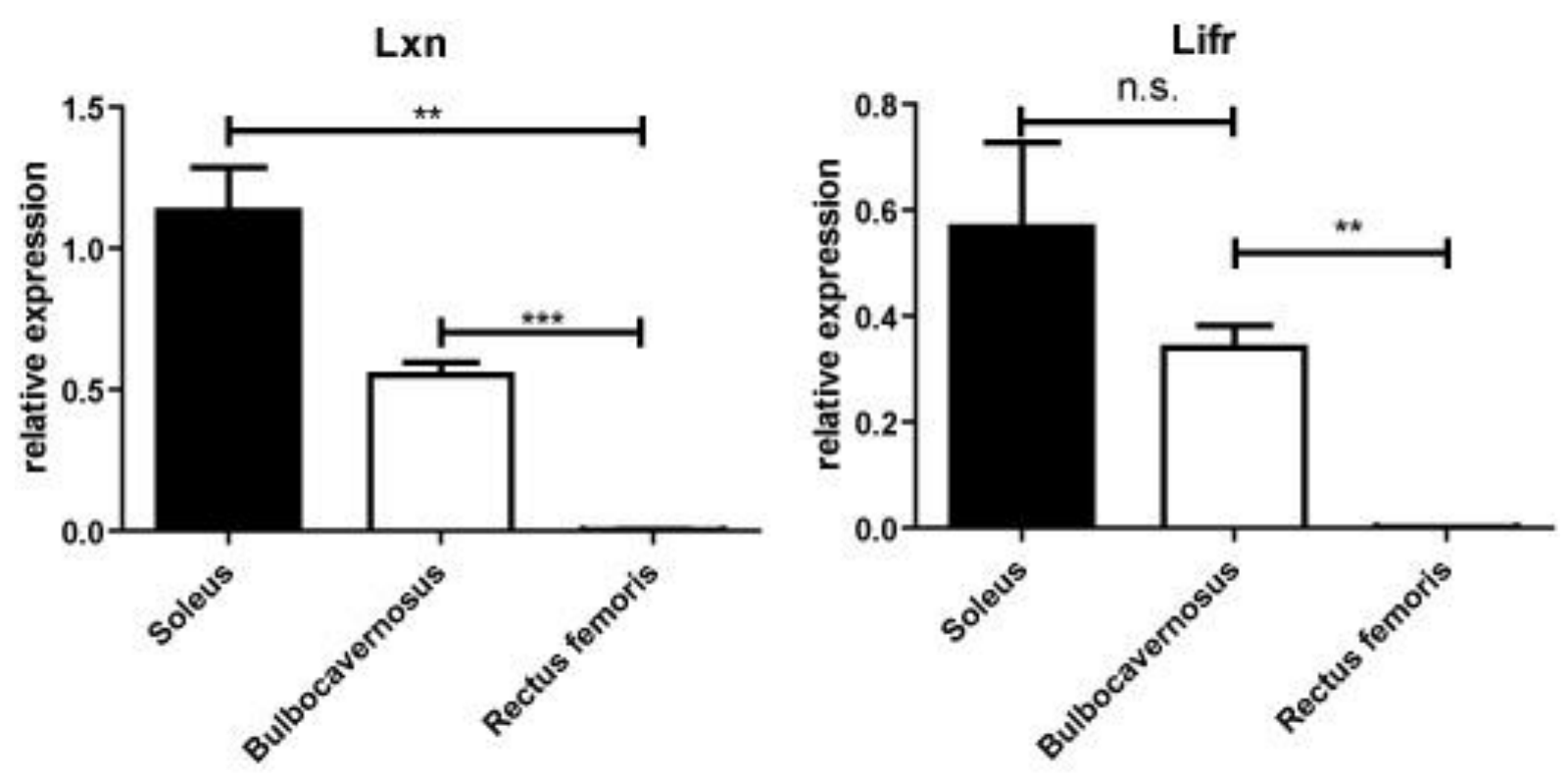

Fig. 24 qPCR of Lxn and Lifr

The graph shows the Relative expression levels of $L x n$ and Lifr in GPCR. BCV and $S$ MNs were compared with expression in RFMNs $(\mathrm{n}=4)$. Expression of alpha tubulin $1 B$ was set as 1 , to which the relative expression levels were normalized. Significant differences are indicated (t-student's test) ${ }^{*} p<$ $0.05,{ }^{* *} p<0.01,{ }^{* * *} p<0.005,{ }^{* * *} p<0.001$

Lifr showed a strong in situ signal in the dorsal part of the ventral horn and a weaker signal in the ventral horn of mouse spinal cord (Fig. 25B). This corresponds to the localization of $S \mathrm{MNs}$ in the retrograde tracing. Surprisingly, no signal was found in the region of $B C V M N s$. This finding could be explained due to the lower endogenous levels of Lifr in BCV MNs, compared to the expression levels in $S \mathrm{MNs}$, reflected in qPCR data for this gene.

Furthermore, in situ hybridization signal for Lxn was also located in the region of the S motor pool (Fig. 25A). Nevertheless, a signal could also be seen in the ventral horn below the position of the RF motor pool and close to the central canal, the region of the BCV motor pool. 

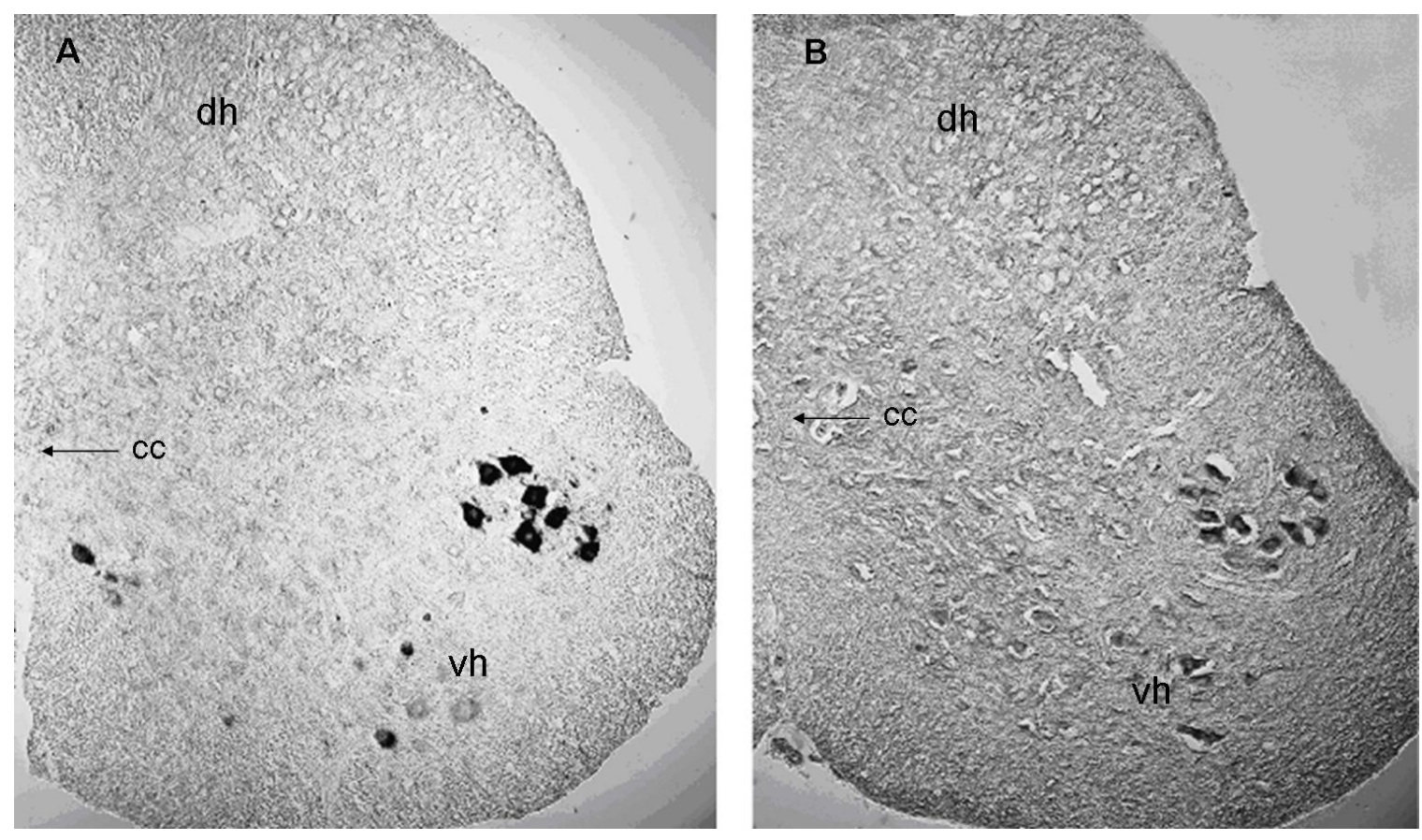

Fig. 25 In situ analysis of $L x n$ and Lifr

(A) Staining of $L x n$ after in situ hibridization where MNs are strongly stained in the dorsal part of the ventral horn, with a weaker staining in ventral horn MNs and MNs close to the central canal, the area of the BCV MNs. (B) Staining of Lifr after in situ hibridization with close restriction of stained MNs to the dorsal part of the ventral horn with minor staining in ventral horn MNs. Pictures were taken from $14 \mu \mathrm{m}$ cryo sections of mouse spinal cord (stage P20).

Even though the expression profile of $R F \mathrm{MNs}$ was clearly different from $\mathrm{BCV}$ and $\mathrm{S}$, we could not find a RF candidate with sufficient high differential expression to serve as a marker for this MN subtype.

\subsection{Relevance of the array data for the vulnerability of MN subtypes}

A high number of candidates were retrieved from the array data and network analysis. Verification of the gene expression levels via qPCR and in situ hybridization was performed on a small set of selected candidates, focusing only on those that showed high differential expression. This study for the first time defined specific molecular markers for different MN subtype-species. 
However, the relatively large and heterogeneous set of differentially expressed gene candidates, did not directly point to specific pathways that would account for the difference in vulnerability of MN subtypes in ALS disease. Therefore, advantage was taken of a genetic screen in the fruitfly Drosophila. In Drosophila, eye-specific overexpression of human TDP-43 (hTDP-43), a protein linked to ALS, elicits mild, but progressive ommatidia loss, causing a visible "rough eye" phenotype (Fig. 26). The screen was performed by knock down of the Drosophila gene-homologues homologues to the candidate genes retrieved from the array data in mouse. If the candidate gene activity influences the neurotoxicity of TDP-43, its knock down would be expected to either worsen or ameliorate the "rough eye" phenotype of the fly. Moreover, other studies have shown that RNAi in flies is an appropriate in vivo model system to screen for neurotoxicity in MNDs (Ding et al., 2003; Lavara-Culebras and Paricio, 2007).

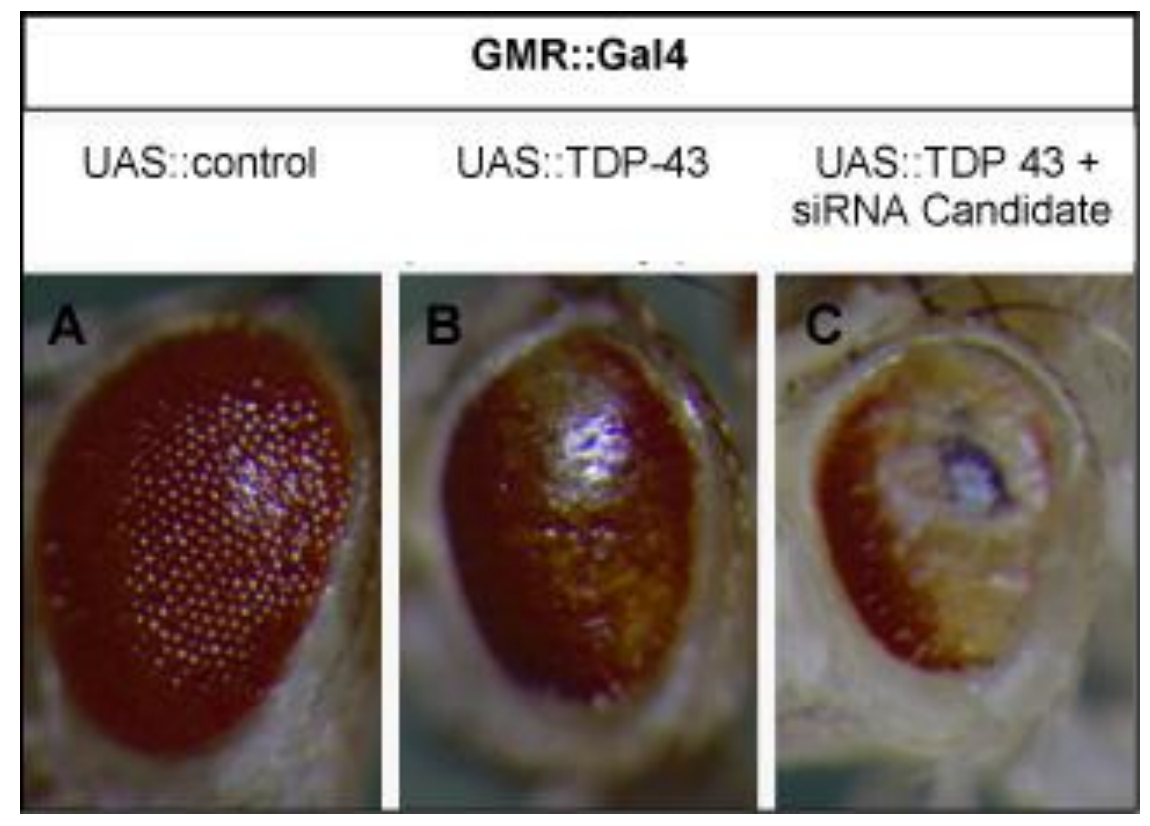

Fig. 26 Rough eye screen

Candidate siRNA co-expression with hTDP-43 in Drosophila eye, mimicking the toxic events in TDP43 mediated ALS toxicity. (A) Expression of a control vector, showing no effect (B) Expression of hTDP-43 caused neurotoxicity. (C) Co-expression of candidate siRNA and hTDP-43 showed enhancement of neurotoxicity (In collaboration with Dr. Aaron Voigt) 
More than 200 candidate genes were screened. From these 200 genes 24 were found to be enhancers and two were suppressors. Furthermore, 19 genes had strong enhancer activity and one was a strong suppressor. Interestingly, most of the candidates with enhanced or suppressed phenotype belong to ubiquitination pathways. Ubxn4 is an example for a candidate that is suggested to be further investigated. The knock down of Ubxn4 that showed higher expression level in $B C V$ and $S$ MNs compared to RF MNs, showed an enhancement of "rough eye" phenotype. Ubxn4 is therefore thought to have a protective function when overexpressed. This suggestion is supported by other studies that showed protective function of Ubxn4 in MND mediated endoplasmatic reticulum stress (ER-stress) (Liang et al., 2006). However, the knock down of small subunit (SSU) processome component (Utp3), a gene that showed high expression in the $S$ MNs, did not have any effect, therefore being an example of a candidate that will not be further investigated (Table 1).

\begin{tabular}{|l|l|l|l|l|l|l|l|}
\hline $\begin{array}{l}\text { Gene } \\
\text { Symbol }\end{array}$ & $\begin{array}{l}\text { Drosophiula } \\
\text { homologue }\end{array}$ & $\begin{array}{l}\text { GT } \\
\text { tdp+siRNA }\end{array}$ & $\begin{array}{l}\text { GMR only } \\
\text { siRNA }\end{array}$ & $\begin{array}{l}\text { TAU + } \\
\text { siRNA }\end{array}$ & $\begin{array}{l}\text { ABETA+ } \\
\text { siRNA } \\
\text { alzheimer }\end{array}$ & $\begin{array}{l}\text { POLY+ } \\
\text { siRNA } \\
\text { huntington }\end{array}$ & gene_assignment \\
\hline Usp47 & CG5486 & suppression & no effect & no effect & no effect & no effect & $\begin{array}{l}\text { ubiquitin specific } \\
\text { peptidase }\end{array}$ \\
\hline Ubxn4 & CG8042 & Enhancement & no effect & no effect & no effect & $\begin{array}{l}\text { Subtle } \\
\text { enhancement }\end{array}$ & UBX domain protein 4 \\
\hline Asah2 & CG1471 & enahncement & no effect & no effect & no effect & $\begin{array}{l}\text { Subtle } \\
\text { enhancement }\end{array}$ & $\begin{array}{l}\text { N-acylsphingosine } \\
\text { amidohydrolase 2 }\end{array}$ \\
\hline Uba3 & CG13343 & $\begin{array}{l}\text { obvious } \\
\text { enhancement }\end{array}$ & no effect & no effect & no effect & no effect & $\begin{array}{l}\text { ubiquitin-like modifier } \\
\text { activating enzyme 3 }\end{array}$ \\
\hline Far1 & CG1443 & enhancement & no effect & no effect & $\begin{array}{l}\text { subtle } \\
\text { enhancement }\end{array}$ & no effect & $\begin{array}{l}\text { fatty acyl CoA } \\
\text { reductase 1 }\end{array}$ \\
\hline Ricalg2 & CG12156 & lethal & no effect & no effect & no effect & no effect & $\begin{array}{l}\text { RAB39B, member } \\
\text { RAS oncogene family }\end{array}$ \\
\hline CG1291 & enhancement & no effect & no effect & no effect & $\begin{array}{l}\text { Subtle } \\
\text { suppression }\end{array}$ & $\begin{array}{l}\text { phosphatidylinositol } \\
\text { binding clathrin } \\
\text { assembly protein }\end{array}$ \\
\hline
\end{tabular}




\begin{tabular}{|l|l|l|l|l|l|l|l|}
\hline Utp3 & CG4202 & no change & no effect & no effect & $\begin{array}{l}\text { subtle } \\
\text { suppression }\end{array}$ & lethal,no effect & $\begin{array}{l}\text { smacessome } \\
\text { component, homolog }\end{array}$ \\
\hline Ap2m1 & CG7057 & $\begin{array}{l}\text { Subtle } \\
\text { enahncement }\end{array}$ & lethal & lethal & no effect & $\begin{array}{l}\text { Subtle } \\
\text { enhancement }\end{array}$ & $\begin{array}{l}\text { adaptor protein } \\
\text { complex AP-2, mu1 }\end{array}$ \\
\hline Cdc5l & CG6905 & lethal & lethal & $\begin{array}{l}\text { subtle } \\
\text { enhancement }\end{array}$ & lethal & Lethal & $\begin{array}{l}\text { cell division cycle 5- } \\
\text { like }\end{array}$ \\
\hline Dhx36 & CG9323 & lethal & lethal & lethal & lethal & Lethal & $\begin{array}{l}\text { DEAH (Asp-Glu-Ala- } \\
\text { His) box polypeptide }\end{array}$ \\
\hline
\end{tabular}

Table 1. Examples of candidate siRNA effects in fly eye disk

The effect of siRNA in Drosophila rough eye screen in the models for well-known neurodegenerative diseases. Only the candidates that either suppress or enhance the phenotype observed in the flies overexpressing TDP-43 are marked with purple. These candidates are thought to be further investigated. (In collaboration with Dr. Aaron Voigt)

\subsection{The Chick embryo as a model system to study ALS pathology}

\section{in vivo}

The genetic screen for suppressors and enhancers of the "rough eye" phenotype caused by hTDP-43 overexpression narrowed the group of candidate genes retrieved from the transcriptome-wide screen to the one with a functional relevance. Ideally, the genes that showed the enhancement of the "rough eye" phenotype when knocked down would have a neuroprotective role when overexpressed. To test this, a vertebrate model system is needed that would allow a high throughput testing of the candidate genes. Chick was chosen as a model, taking advantage of the temporally compressed neuromuscular maturation typical for precocial species. This model thus facilitates ready experimental access to mature vertebrate motor neurons through in ovo transfection.

First, the impact of TDP-43 expression in chick motor neurons was examined. In this approach a novel expression system was used achieving stable neuron subtypespecific transgene expression in chick in vivo (see materials and methods). This 
system was based on tol2-transposon-mediated stable genomic insertion (Kawakami, 2007), combined with restricted transgene activation via postmitotic MN-specific Cre recombination mediated by an $\mathrm{Hb9::Cre} \mathrm{driver} \mathrm{construct.} \mathrm{The} \mathrm{TDP-43} \mathrm{long-term}$ expression constructs were injected into the neural tube of E3 chick embryos and transfected via electroporation (Fig. 27). The electroporation leads to transfection of only one half of the spinal cord. With this system, one obtained the control "wild type" within the same sample. This system facilitated specific and stable N-terminally V5 peptide-tagged TDP-43 expression in the late-stage chick MN (Fig. 27).

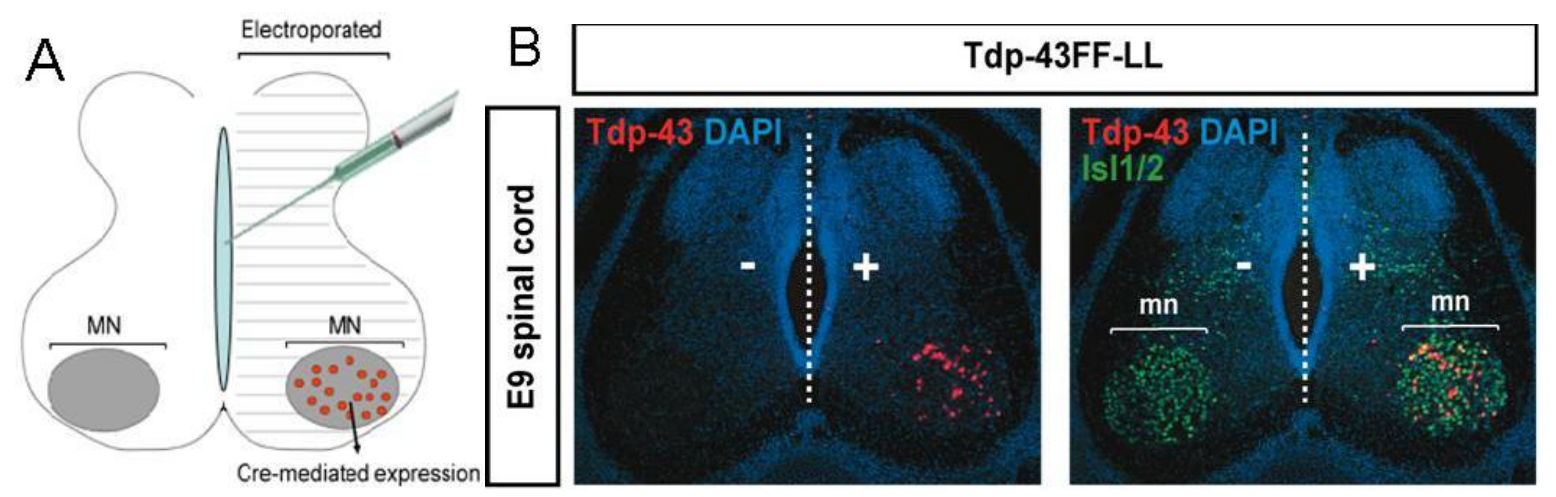

Fig. 27 Stable expression of TDP-43 in chick spinal cord MNs

(A) The scheme shows the injection site for the DNA mixture of TDP-43 expression vector, $\mathrm{Hb9::Cre}$ and transposase. (B) TDP-43 expression in MNs on the electroporated + site (red) overlaps with IsI $1 / 2$ (green) immunostaining as a MN marker. DAPI was used as nuclear marker (blue).

We next tested the impact of ALS-linked variants of TDP-43 on MN survival. This included human full length TDP-43 ${ }^{\mathrm{WT}}$, a full length version of TDP-43 with a point mutation found in human patients (A315T), a deletion mutation of the nuclear localization signal 'TDP- $43^{\triangle N L S}$, a deletion mutation in the RNA-binding motif 'TDP$43^{\mathrm{FFLL}}$, a truncation of the N-terminus 'TDP- $43^{\mathrm{CTF}}$, a truncation of the C-terminus 'TDP- $43^{\text {NTF' }}$ and a double deletion in the nuclear localization signal and the RNAbinding motif 'TDP-43 ${ }^{\Delta N L S F F L L '}$ (Fig. 28). 


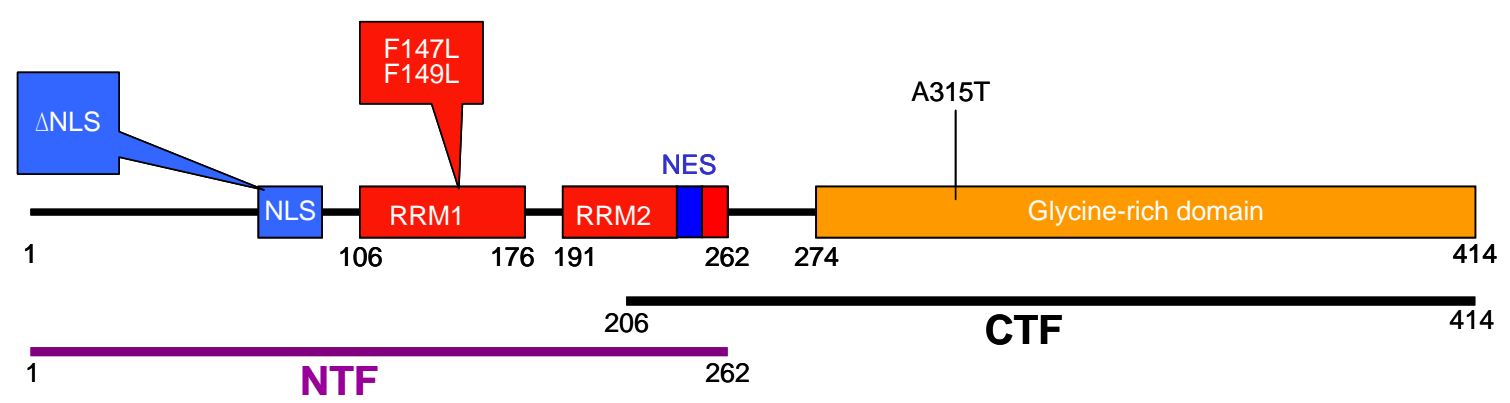

Fig. 28 Scheme of TDP-43 with indications of used variants

TDP-43 consists of a nuclear localization signal (NLS), the RNA recognition motifs 1, 2 (RRM1,RRM2), a nuclear export signal (NES) and a Glycine-rich domain. Variants of TDP-43 used in the overexpression experiments were the $\mathrm{N}$-terminal fragment (TDP- $43^{\mathrm{NTF}}$ ), the $\mathrm{C}$-terminal fragment (TDP- $43^{\mathrm{CTF}}$ ), a full length variant with deletion of NLS (TDP- $43^{\mathrm{ANLS}}$ ), a amino acid substitution resulting in loss of RNA-binding activity (TDP- $43^{\mathrm{F} 147 \mathrm{~L}}$, TDP- $43^{\mathrm{F} 149 \mathrm{~L}}$ ) and a point mutation found in human ALS patients (TDP-43 ${ }^{\mathrm{A} 315 \mathrm{~T}}$ ).

When overexpressed in chick, TDP-43 ${ }^{\mathrm{WT}}$, TDP-43 ${ }^{\mathrm{A} 315 \mathrm{~T}}$ and TDP-43 ${ }^{\mathrm{FFL}}$ showed a strict localization to the nucleus. Only strongly overexpressing cells displayed a mislocalization into the cytosol (Fig. 29). Nevertheless, TDP-43 ${ }^{\Delta N L S}$ showed the expected mislocalization into the cytosol as well as TDP $-43^{\triangle N L S F F L L}$ and TDP- $43^{\mathrm{CTF}}$ (Fig. 30), that is missing the NLS signal due to truncation. Furthermore, TDP $-43^{\mathrm{NTF}}$ showed a main localization in the nucleus and a weak distribution into the cytosol (Fig. 30). Interestingly, findings in chicken exactly mirrored the data obtained from the Drosophila experiments proving compatibility of the same disease model in two different species (Fig. 29). 


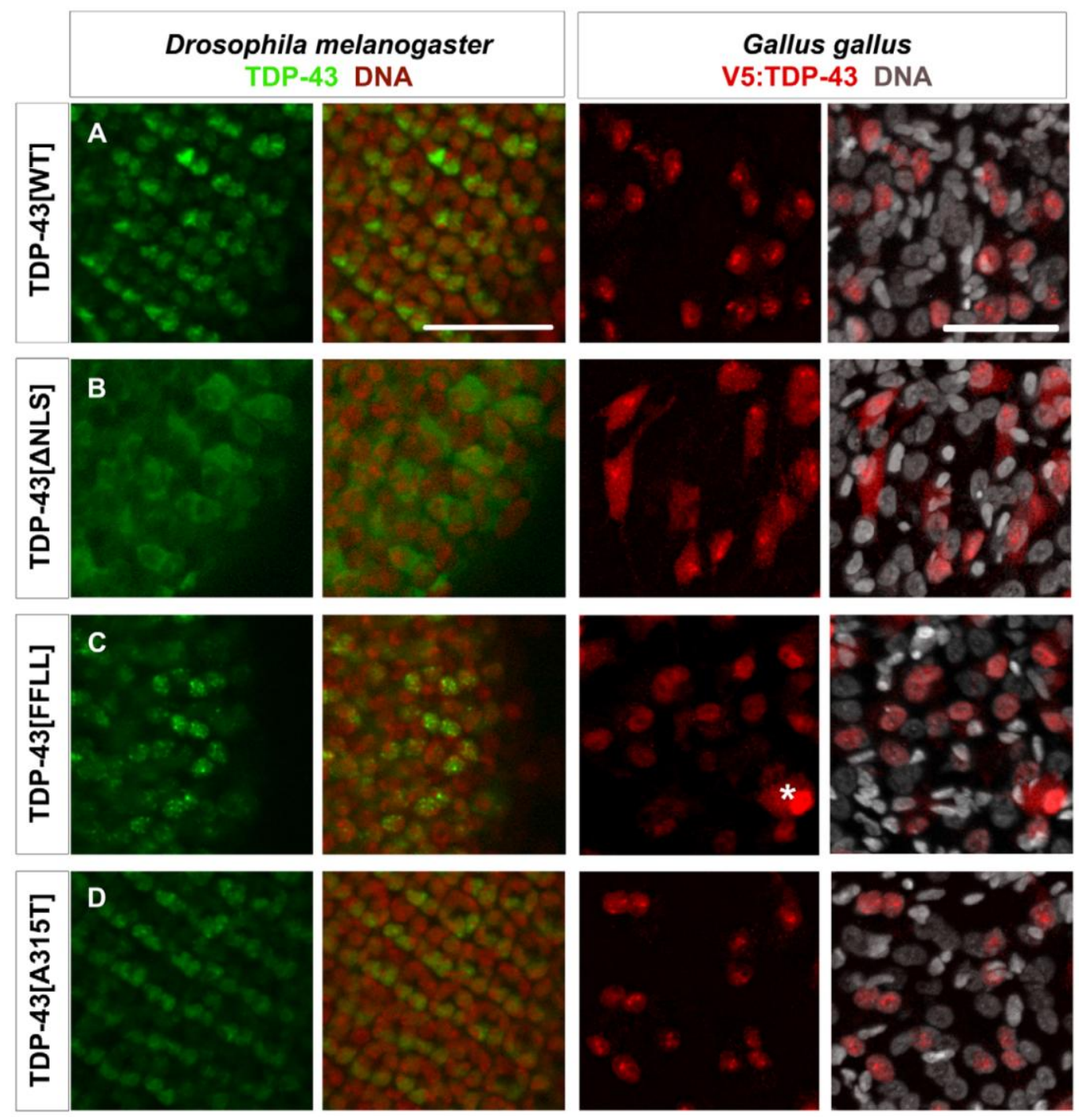

Fig. 29 Localization of TDP-43 in Drosophila melanogaster and Gallus gallus.

Confocal sections of eye imaginal discs from Drosophila larvae (left panel) and motor neurons from Gallus (right panel) expressing indicated TDP-43 variants. To be able to discriminate between the two in vivo systems, ectopic TDP-43 in Drosophila is shown in green, whereas TDP-43 in Gallus is shown in red. Subcellular localization of the different TDP-43 variants was found to be identical between fly and chick. TDP-43 ${ }^{\mathrm{WT}}$ (A) localized mainly to the nucleus, while TDP-43 ${ }^{\triangle N L S}$ (B) was found predominantly in the cytoplasm. TDP- $43^{\mathrm{FFLL}}(\mathrm{C})$ and TDP- $43^{\mathrm{A} 315 \mathrm{~T}}$ (D) displayed a nuclear distribution. Only cells with very high expression levels of usually nuclear TDP-43 displayed a detectable cytoplasmic staining (example in case of TDP-43 ${ }^{\mathrm{FFLL}}$ marked by asterisk). DNA was stained with SytoxH Orange (fly, red) or DAPI (chick, white). Scale bar indicates $50 \mathrm{~mm}$. Neuronal expression was mediated by elav::Gal4 (flies) or Hb9::Cre (chick). (In collaboration with Dr. Aaron Voigt)

Unilateral expression of hTDP-43 ${ }^{\mathrm{WT}}$ in postmitotic motor neurons for 6 days, triggered loss of roughly $30 \%$ Isl $1 / 2$ positive spinal motor neuronsrelative to the non- 
transfected control hemicord (Fig. 31). Significant, but comparatively milder MN loss was observed upon expression of TDP- $43^{\triangle N L S}$ and TDP $-43^{A 315 T}$ forms. Relative to the

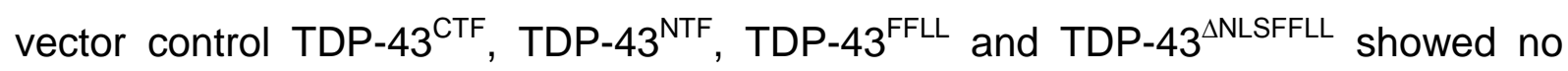
toxicity at all. Based on these results, one can speculate that loss of MNs was restricted to full length TDP-43 with a functional RNA-binding motive (Fig. 31). Loss was further preceded by transient increase in Caspase-3 positive motor neuron numbers, mirroring the relative impact of TDP-43 variants on overall reduction of MN numbers. This suggests that $\mathrm{MN}$ loss occurred at least in part via caspase-dependent apoptotic elimination. 


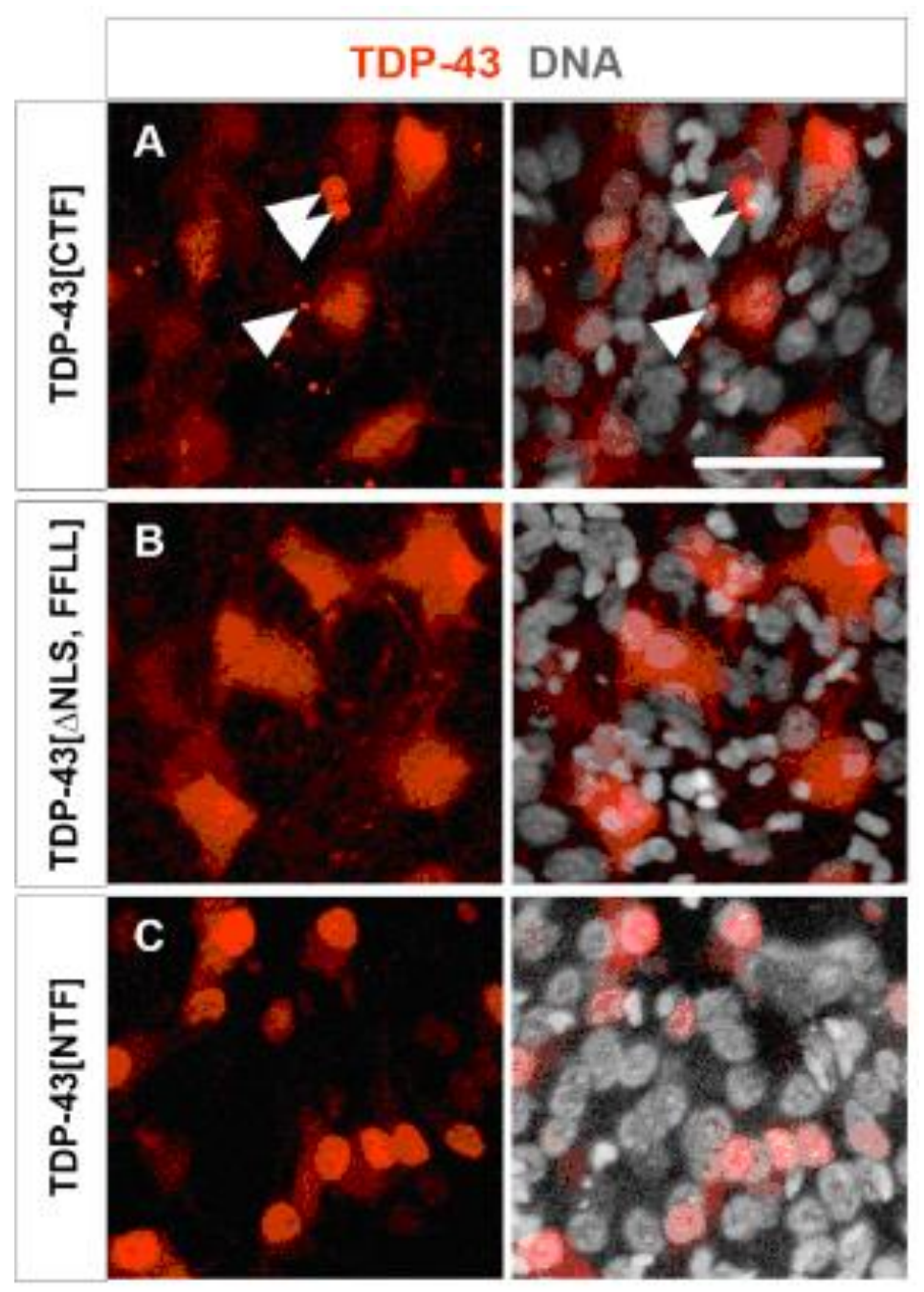

Fig. 30 Subcellular localization of additional TDP-43 variants in Gallus

Subcellular localization of TDP-43 variants (red) in E9 chick motor neurons (large DAPI+ nuclei: white). In analogy to cytosolic and nuclear localization observed in flies, TDP-43 ${ }^{\mathrm{CTF}}$ (A) in chick localized alike and displayed frequent cytosolic foci (arrowheads). To test if the lack of RNA-binding also abolishes toxicity of cytoplasmic TDP-43, we combined RNA-binding deficient FFLL with the mutated NLS and generated TDP-43 $3^{\triangle N L S, F F L L}$. As observed for TDP-43 ${ }^{\triangle N L S}$, TDP- $43^{\triangle N L S, F F L L}$ localized predominantly to the cytoplasm (B). Thus, interference with RNA-binding of TDP-43 did not alter localization. Scale bar indicates $50 \mu \mathrm{m}$. 

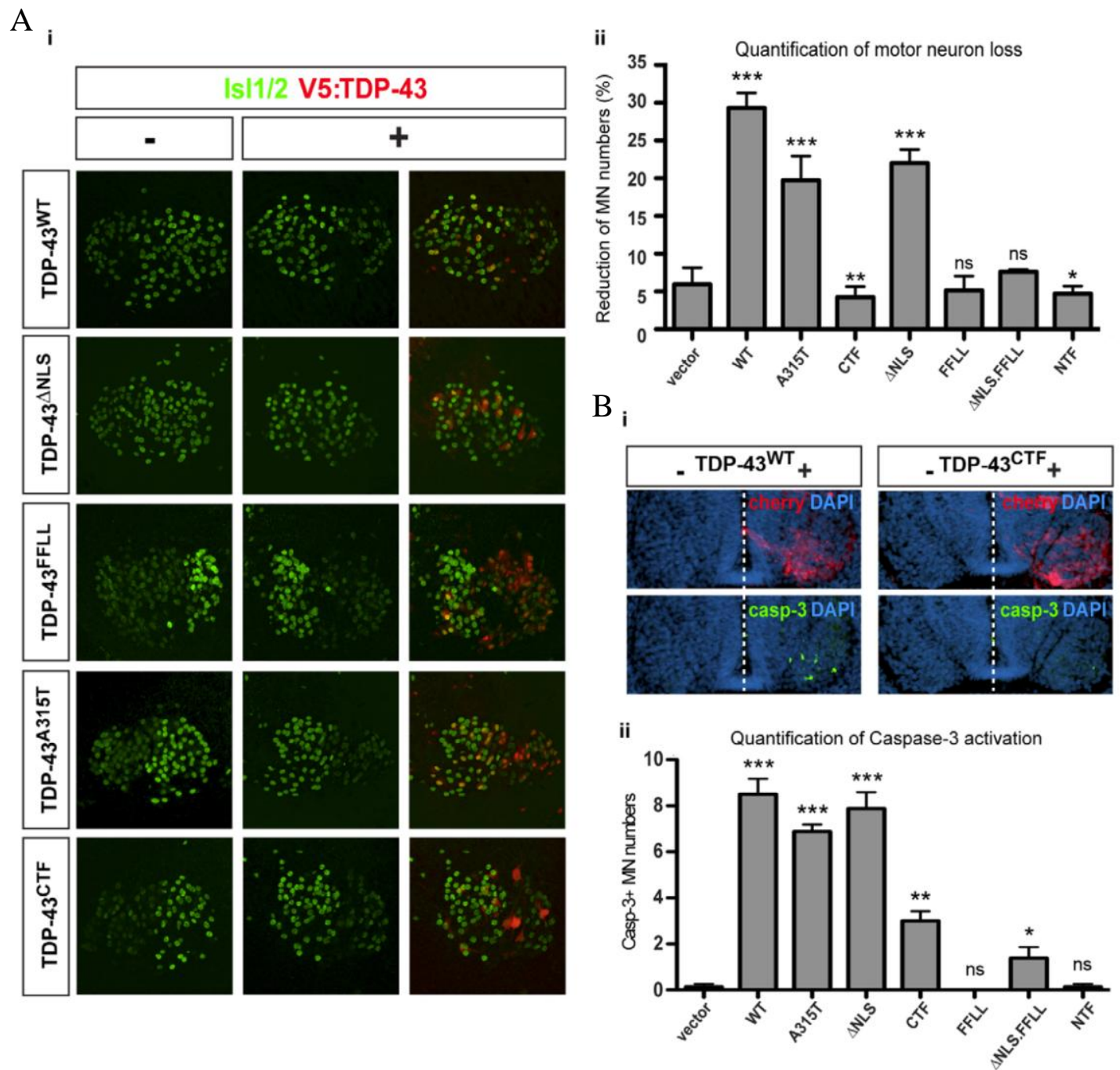

Fig. 31 RNA-binding activity is required for TDP-43-mediated motor neuron loss in chick

(A) [i] Examples of thoracic motor columns (Is/1/2+ motor neurons: green) upon TDP-43 variant expression (red). [ii] Quantification of motor neuron loss upon TDP-43 variant expression over all obtained sections (in "-" versus "+" hemicord). Differences relative to control (t-student's test) are indicated. (B) [i] Activated Caspase-3 (green) detected in E5 motor neurons upon TDP-43 ${ }^{\text {WT }}$ expression (indicated by IRES-cherry bi-cistronic reporter: red). Compared to E5 motor neurons expressing TDP-43 ${ }^{\mathrm{WT}}$, little activation of Caspase- 3 was detected upon TDP-43 ${ }^{\mathrm{CTF}}$ expression. [ii] Quantification of Caspase-3 activation in motor neurons of transfected hemicords versus vector control. Significant differences are indicated (t-student's test - relative to control). ${ }^{*} p, 0.05 ;{ }^{* *} p, 0.01$; ${ }^{* * *} \mathrm{p}, 0.001$; ns not significant.

In conclusion, the approach showed that we obtained a powerful and precise tool, appropriate for in vivo impact investigations of candidate gene expression in motor neurons. 


\subsection{Verifying the role of candidate genes in protection from TDP-43 induced neurotoxicity}

As mentioned above, the candidates that showed the enhancement of the "rough eye" phenotype in Drosophila overexpressing hTDP-43 ${ }^{\mathrm{WT}}$ when knocked down, would ideally have a neuroprotective role when overexpressed. To test this, the candidate gene should be stably expressed in our chick model system for TDP-43 induced neurotoxicity. For this, the vector for long-term expression was remodelled so that it co-expresses a candidate gene with TDP-43 fused to GFP (Fig. 32). This allows the stable expression of the candidate gene together with the visualization of TDP-43. This should give us new insights how our candidates affect ALS-like TDP43 neurotoxicity in a vertebrate model system.

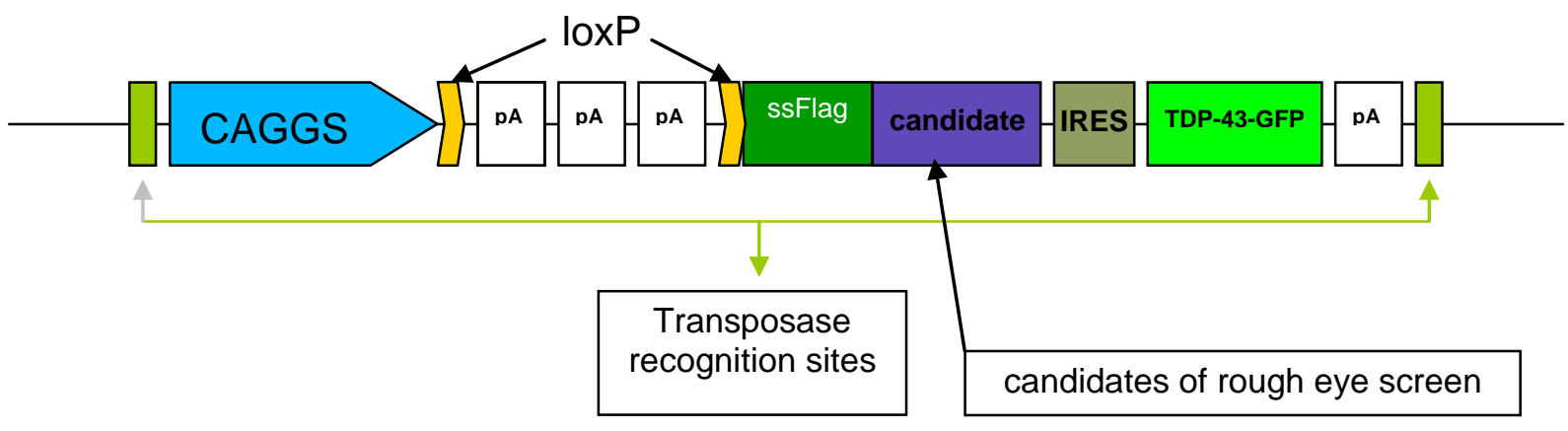

Fig. 32 Modified long-term expression vector

The expression vector used in A. Voigt et al. 2010 was modified for coexpression of candidate gene with TDP-43. (For original vector see material and methods) 


\section{Discussion}

Motor neuron diseases (MNDs) like amyotrophic lateral sclerosis (ALS) have been investigated in a variety of approaches. These studies have lead to a range of alternative and often conflicting models for the disease-causing mechanism/s. A common denominator of MNDs is the vulnerability of MNs. Several lines of evidence strongly indicate intrinsic differences between distinct types of MNs that render them more or less sensitive or resistant towards neurodegeneration. However, the mechanistic basis of these differences remains unknown.

\subsection{Genetic differences between MN subtypes}

The identification of genes potentially involved in mediating MN vulnerability vs. resistance was performed by isolating MNs that differ in their vulnerability towards MNDs. Since most motor pools innervating a distinct muscle consist of a variety of MN subtypes, muscles were chosen which are innervated mainly by one type of MN. For instance, the $M$. Rectus femoris $(R F)$ as a muscle consistent of fast muscle fibres and thereby innervated by fast MNs. The motor pool innervating the $R F$ represents highly susceptible MNs, since motor neurons innervating the $R F$ are known to die early in disease progression of ALS. Furthermore we chose the M. Soleus $(S)$ muscle. It consists of slow muscle fibres and is therefore innervated by slow MNs. Slow MNs have been shown to be affected in later stages of disease progression, whereas they should represent the relatively resistant MNs (Pun et al., 2006). Resistant MNs could be shown to innervate the M. Bulbocavernosus (BCV) and seem to be unaffected throughout the whole disease process (Hamson et al., 2002). Among the pool of genes selectively expressed in $B C V$ and $S$ MNs was expected to 
find factors involved in the intrinsic resistance of these MN subtypes. Alternatively, the specific expression of certain factors in vulnerable MNs may render these neurons particularly receptive for ALS-causing effectors.

The expression profiles of three MN subtypes were compared and highly differential gene signatures were gained. These findings showed similarities in expression profiles between $B C V$ and $S M N s$, while, the gene expression found in $R F M N s$ was highly differential.

The array data was analysed in two directions:

1. Establishing of marker genes for BCV and $\mathrm{S}$ via $\mathrm{QPCR}$ and in situ hybridization

2. Revealing mediators of resistance via functional analysis in "rough eye" screen in Drosophila and in silico pathway analysis

3. Establishing of a stable chick gain of function system for candidate impact testing on MN survival

The gene Cartpt was identified as a specific marker for resistant BCV MNs, since it showed more than threefold higher expression level in this MNs compared to $R F$ or $S$ MNs. Furthermore, the in situ hybridization showed the expression restricted to $B C V$ MNs. Uts2 was selected as a marker for relatively resistant $S$ MNs, based on the expression data. $L x n$ and Lifr were identified and confirmed as genes that are specific for the less vulnerable $B C V$ and $S$ MNs. Based on the qPCR data, their expression profile positively correlates with the disease-resistance levels of the different MN subtypes.

The "rough eye" screen was based on an enhancer/suppressor model system mimicking toxics events in ALS by TDP-43 expression. In parallel, an in silico pathway analysis of the candidates was performed, thereby candidates that had no 
fly homologue were also taken into account. Possible mediators of resistance have to be further investigated in the future. Of particular interest is their impact in a chick model system for ALS-like TDP-43 toxicity. Subsequently, candidates showing suppression of TDP-43-mediated toxicity in chick will be tested on MN loss and disease progression in ALS mouse models (Fig. 33).

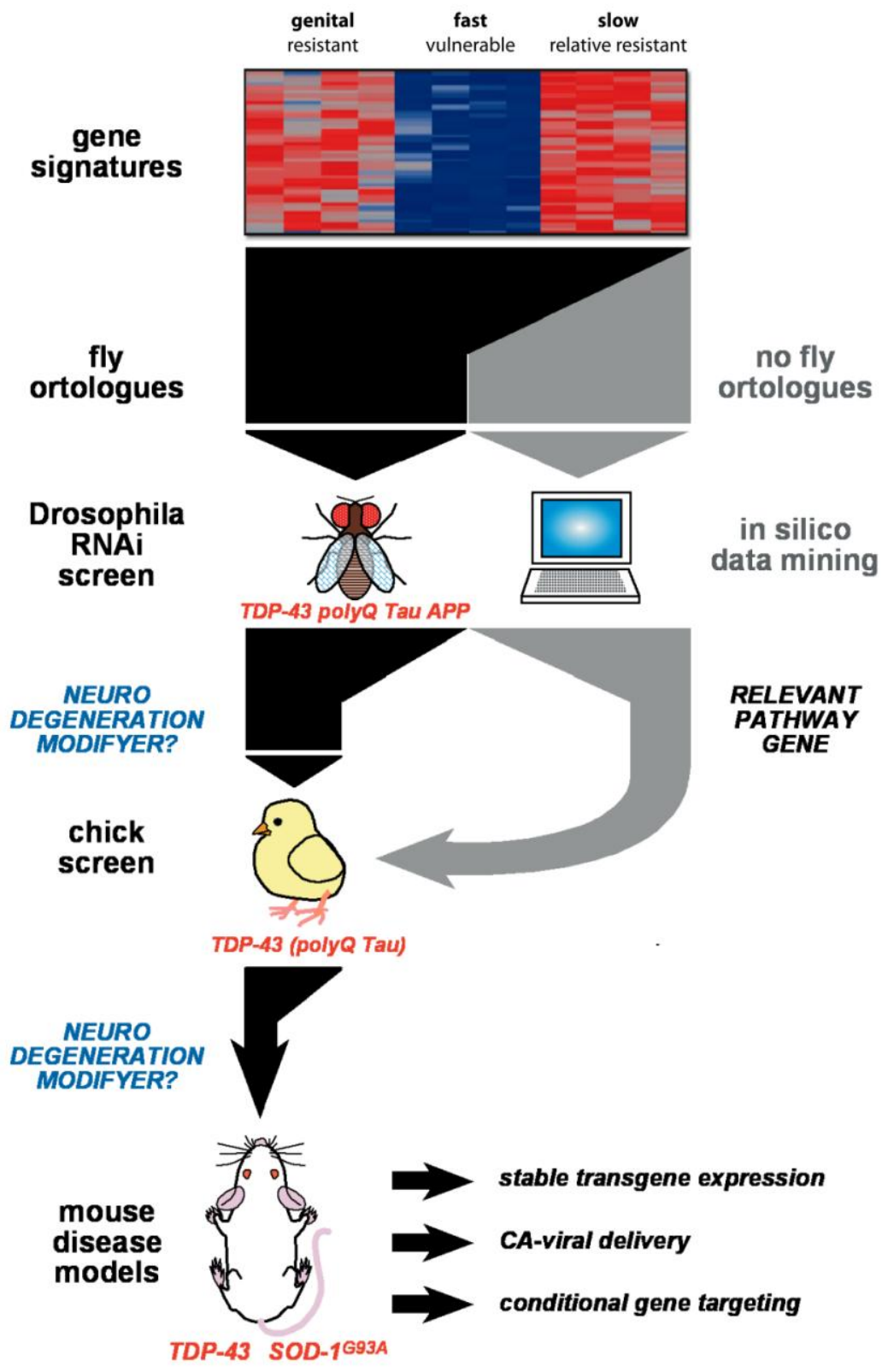

Fig. 33 Workflow chart 


\subsection{Relevance of differential gene expression to neuroprotection}

\subsubsection{CART}

Apoptotic events were found in fALS in cultured mutant SOD1 transgenic neuronal cells (Pasinelli et al., 1998; Rabizadeh et al., 1995), spinal cord of human patients and in SOD1 mutant mouse models (Li et al., 2000; Pasinelli et al., 1998). The apoptosis precursors caspase- 1 and caspase- 3 have been found activated in these cases, whereas the inhibition of caspase-1 prolonged survival in $S O D 1^{G 93 A}$ mice (Friedlander et al., 1997; Li et al., 2000). This thesis shows that hTDP-43 overexpression leads to $\mathrm{MN}$ loss that was preceded by activation of caspase-3. This suggests that MN loss in both SOD1 and TDP-43-linked ALS is at least in part mediated by caspase-dependent apoptotic elimination.

Apoptosis can be triggered by various events including excitotoxicity or mitochondrial dysfunction which have all been implicated in ALS. Therefore, protection against apoptotic effects on neurons was also tested in ALS models. Mediators of cell survival are for example the anti-apoptotic protein $\mathrm{Bcl}-2$, phosphatidylinositol 3-kinase (PI3-K), pp90 ribosomal S6 kinase (RSK), extracellular signal regulated protein kinase (ERK) and cAMP-response-element-binding protein (CREB), which have been found to be involved in blocking apoptotic pathways (Yuan and Yankner, 2000). Bcl-2 overexpression in particular was found to act neuroprotective in SOD1 mutant mice (Kostic et al., 1997). Moreover, ERK was found to activate RSK and RSK therefore CREB, which further activates $\mathrm{Bcl}-2$ and activates transcription of pro-survival factors. Interestingly, in this thesis it was found that ERK was highly expressed in $B C V$ and $S \mathrm{MNs}$, but this observation still needs to be confirmed. Since glutamateinduced excitotoxicity is thought to be part of SOD1-mediated ALS toxicity, ERK and PI3-K have been shown to act in a neuroprotective manner in glutamate-induced 
apoptotic cell death in hippocampal neurons (Almeida et al., 2005), which could indicate a possible connection to neurotoxicity in ALS.

Previous studies identified the Cocaine- and amphetamine-regulated transcript prepropeptide (Cartpt/Cart) as an activator of the ERK signalling pathway. This interaction was shown to be very important in protection against cerebral ischemia.

Furthermore, Cart has been shown to interact with the subunit B of the mitochondrial enzyme succinate-dehydrogenase (SDHB) (Mao et al., 2007), which is a subunit of the complex II of electron transport chain in mitochondria. This interaction increased complex II activity in purified mitochondria, as well as in cultured neurons and thus increased ATP production. Furthermore, interaction of Cart with SDHB protected neurons against oxygen-glucose deprivation (Mao et al., 2007). Together, these results suggest a neuroprotective role of Cart.

It has been shown that in ALS patients as well as in SOD1 mutant mouse complex I abundance and activity is decreased (Jung et al., 2002). In a study performed by Zhao et al., a ketogenic diet changed the clinical and biological alternations in SOD1 mutant mouse (Zhao et al., 2006). It has been demonstrated that ketone bodies increase ATP production through complex II, thus bypassing the reduced function of complex I. In light of this data, one could speculate that neuroprotective role of Cart may be mediated in a similar manner, through increasing the activity of complex II.

In the context of these studies, the findings of highly differential expression of Cart in MNDs resistant $B C V$ MNs could indicate an involvement of Cart in the intrinsic neuroprotection in these MNs. 


\subsubsection{Lxn}

Latexin ( $L x n)$ is a cytosolically localized protein and the only protein known in mammals with carboxypeptidase inhibition activity, which implicates it in protein turnover regulation (Aagaard et al., 2005; Garcia-Castellanos et al., 2005; Normant et al., 1995). Furthermore, Lxn is involved in stem cell aging and lifespan and is thought to prevent aggregation-mediated toxicity in old stem cells and to preserve their functionality (Liang and Van Zant, 2008). Also interesting is the structural similarity to the cystein protease inhibitor, cystatin $\mathrm{C}$, which could indicate similar functions of Lxn. Cystatin C was demonstrated to act protective in Alzheimer's disease by overexpression in disease mouse models (Kaeser et al., 2007; Mi et al., 2007), by diminishing aggregates of amyloid- $B$. Endogenous Lxn was moreover found to be downregulated in a mouse model of Alzheimer's disease (Mirnics et al., 2003). While Lxn is thought to have similar functions as cystatin $\mathrm{C}$, a similar protective role in Alzheimer's disease could be suggested when Lxn is overexpressed. Since deposition and aggregation is a hallmark of several neurological diseases, especially in ALS, Lxn could hypothetically act neuroprotective when overexpressed in MNs, due to its role in protein turnover. Furthermore, Lxn was shown to be absent or decreased in expression in human leukemia/lymphoma cell lines and patients (Liang and Van Zant, 2008), suggesting a protective function when overexpressed, which still has to be confirmed. A higher expression of Lxn could be shown in relatively resistant $S \mathrm{MNs}$ and resistant $B C V \mathrm{MNs}$, supporting the idea of a protective function of Lxn. 


\subsubsection{Uts2/Uts2d}

Two out of the three members in the Urotensin II family were found to be highly expressed in S MNs, urotensin 2 (Uts2) and urotensin 2 domain containing (Uts2d). Uts2d has been shown to bind and activate the third member of the Urotensin II family, urotensin 2 receptor (Uts2r) (Jiang et al., 2008). Moreover, Uts2 and Uts2r have been reported to alter glucose metabolism and insulin resistance, a hallmark of the human disease type 2 diabetes mellitus (T2DM). Highly elevated levels of Uts2 and Uts2r have been found in tissue of human diabetes patients (Langham et al., 2004). Related to Uts2 in MNs it is known that its expression is downregulated in androgen expressing neurons (Pelletier et al., 2002).

\subsubsection{Lifr}

The leukemia inhibitory factor receptor (Lifr) has been shown to be activated by Lif (Davis et al., 1993; Gearing et al., 1991). Most prominent functions of Lif protein are differentiation of M1 monocytic leukaemia cells and differentiation inhibition of embryonic stem cells. Interestingly, Lifr has been found to protect oligodendrocytes from cell death in multiple sclerosis (MS) mouse model (Butzkueven et al., 2002). Hallmarks of the disease are demyelination and inflammatory infiltration in certain areas of the central nervous system (Lucchinetti et al., 2000).

The mechanisms of Lifr's protective role are not yet fully understood, but several studies implicate a way through well known cell survival pathways. In the spinal cord of MS disease mouse models, Lifr is activated via exogenous Lif, followed by activation of STAT-3 (Butzkueven et al., 2002). STAT-3 was shown to activate transcription of anti-apoptotic proteins, like Bcl-2 and Bcl-xl in plasma and NIH3T3 cells (Bowman et al., 2000). Furthermore, activation of ras-MAP kinase pathway (ERK pathway) as well as initiation of the nuclear factor-kB (NF-kB) mediated 
survival mechanism through activation of PI3-K in neural cells (Fukunaga and Miyamoto, 1998; Middleton et al., 2000; Segal and Greenberg, 1996). NF-kB and $\mathrm{Bcl}-2$ were found to be expressed in demyelinating areas of human MS patients (Bonetti et al., 1997; Bonetti et al., 1999). Other targets of STAT-3 are the cytokines IFN- $\gamma$ and TNF- $\alpha$. These cytokines are confirmed to induce oligodendroglial or fibroblast cell death and are inhibited by STAT-3 (D'Souza et al., 1996; Vartanian et al., 1995). Lif can directly inhibit cytokine signalling by transcription activation of the suppressor of cytokine signalling (SOCS) proteins SOCS-1 and SOCS-3 (Turnley and Bartlett, 2000), whereas SOCS-1 has been shown to inhibit TNF-induced apoptosis in fibroblasts (Morita et al., 2000). Moreover, Lifr and Lif expression were found to be increased in the hippocampus of Alzheimer's disease as well as in the anterior cingulated cortex of Parkinson's disease patients (Soilu-Hanninen et al.). Whereby, Lif was indicated to protect neurons from Aß-induced toxicity in Alzheimer's disease (Rensink et al., 2002). Interestingly, a Lif knock out showed significant loss of MNs in postnatal mice (Sendtner et al., 1996), whereas Lifr depletion was lethal due to severe prenatal MN loss (Li et al., 1995), which showed an important function of Lif and Lifr in the viability of MNs (Sendtner et al., 1996). In addition, Lif production increased rapidly after nerve lesion in rats (Banner and Patterson, 1994; Curtis et al., 1994), leading to the hypothesis of neuroprotective functions of Lif.

All of these studies strongly indicate a neuroprotective role of Lifr, consistant with findings of this thesis of elevated Lifr expression in relatively resistant and resistant MNs. Furthermore, Lifr seems to be involved in survival pathways similar to those activated by Cart, suggesting a common mechanism of neuroprotection in $B C V$ and S MNs. It could be suspected that Cart may enhance protective effects mediated by Lifr, thus explaining the difference in resistance between $S$ and $B C V$ MNs. 


\subsubsection{Ubxn4}

Misfolded and misassembled proteins undergo degradation at the endoplasmatic reticulum (ER) in a process called ER-associated protein degradation (ERAD). This is a process where proteins are transported from the ER into the cytosol, where they are degraded by the proteasome (Hirsch et al., 2009; Kanehara et al., 2007; Vembar and Brodsky, 2008). Several studies suspected ERAD to be part of human disease processes, as there were found mutations in several proteins leading to their misfolding in the ER, with subsequent activation of ERAD ((Brodsky, 2007; Schroder and Kaufman, 2005; Zhao and Ackerman, 2006). Under normal conditions ERAD serves as a safety mechanism, protecting the cell from damaged and possible toxic proteins. Impaired functions of ERAD therefore could lead to accumulation of these proteins and cause toxic events in the cell. Furthermore, mutant proteins found in Huntington's disease and ALS have been demonstrated to interfere with ERAD thereby causing ER-stress (Duennwald and Lindquist, 2008; Nishitoh et al., 2008). Interestingly, valosin-containing protein (p97/VCP), has been recently found to be mutated in MNDs such as fALS, where it interacts with TDP-43 in human patients (Johnson et al., 2010). Mutations in VCP have been shown to lead to disposition and aggregate forming of TDP-43 in MNDs (Shaw, 2010). In respect to its physiological role, VCP has been demonstrated to be recruited to the ER for ERAD (Kostova et al., 2007). VCP acts as an extractor for ERAD substrates by binding to ER-located proteins. Candidate for this assisting binding is among others the UBX domain protein 4 (Ubxn4). The extraction process of damaged proteins is accompanied by tagging the protein with a polyubiquitin chain by ubiquitin ligases (Kostova et al., 2007), representing a signal that determines the protein to be degraded by the proteasome. An ubiquitin-protein ligase (Ufd2/Ube4b) is responsible for tagging of the substrates with these ubiquitin chains and is recruited to ERAD by binding to VCP 
(Richly et al., 2005). Liang et al. have shown that Ubxn4 is binding to VCP and increases ERAD efficiency when overexpressed in vitro. Therefore, knock down of Ubxn4 caused a complete inhibition of ERAD, suggesting a key role for Ubxn4 in ERAD mechanisms (Liang et al., 2006). When treated with ER-stress inducing agents, cells in culture showed higher levels of Ubxn4, confirming the protective character of this protein. In addition, Ubxn4 was found to be elevated in neuropathological brain regions of human Alzheimer's disease patients, suggesting that Ubxn4 is involved in the degradation of $A B$ aggregates (Liang et al., 2006). Moreover, another interaction partner of VCP, ataxin-3 (Atxn3) was found to decrease ERAD activity. Atxn3 interacts with VCP in a way that prevents retrotranslocation of targeted proteins from ER to the cytosol in vitro (Zhong and Pittman, 2006). Ultimately this leads to aggregation of ubiquitylated proteins in the ER. Extended polyglutamine stretches of Atxn3 have been shown to lead to a neurodegenerative disease similar to Huntingtin's disease (Zoghbi and Orr, 2000). This thesis provided evidence for a modifier activity of Ubxn4 in ALS and Huntington's disease, where it was shown to enhance TDP-43 and polyglutamine (PQ)-mediated toxicity upon its knock out in the rough eye screen. Thus, Ubxn4 shows a direct link between PQ and TDP-43-mediated neurotoxicity.

Taken together, all these studies strongly suggest a protective role of Ubxn4 by degradation through ERAD, preventing toxic levels of misfolded or damaged proteins. In support to this idea, expression of Ubxn4 has been found to be elevated in the relatively resistant and resistant MNs in this work. 


\subsubsection{Calb2/parvalbumin}

MNs have been suggested to lack important $\mathrm{Ca}^{2+}$ buffering proteins like parvalbumin and calbindin 1 (De Maria et al., 1994; Ince et al., 1993). Furthermore, it has been shown that MNs have a high number of $\mathrm{Ca}^{2+}$ permeable AMPA glutamate receptors (Shaw, 2005), allowing fast $\mathrm{Ca}^{2+}$ influx. Hence, a highly functional $\mathrm{Ca}^{2+}$ buffering system for compensation is essential for MNs. Increased levels of $\mathrm{Ca}^{2+}$ have been found in ALS patients, as well as in cell lines and SOD1 mutant mouse models. These elevated levels have been proposed to lead to mitochondrial perturbations, since mitochondrial calcium buffering capability was found to be impared in $S O D 1^{G 93 A}$ mouse model MNs and neuroblastoma cell cultures (Jaiswal et al., 2009). Subsequently, this could interrupt the supply of the $\mathrm{Na}^{+} / \mathrm{K}^{+}$ATPase with ATP, leading to accumulation of $\mathrm{Na}^{+}$in the cytosol and cause hyperexcitability of the MN. In order to remove the accumulating $\mathrm{Na}^{+}$, more $\mathrm{Ca}^{2+}$ would be imported and raise activation of degradative enzymes like proteases (Cheah et al., 2010). Furthermore, in $S O D 1^{G 93 A}$ mice, high levels of $\mathrm{Ca}^{2+}$ appear to be involved in inducing apoptosis by release of mitochondrial cytrochrome c into the cytosol (Kirkinezos et al., 2005; Pasinelli et al., 2004; Takeuchi et al., 2002), which is consistent with the finding of reduced expression of Calretinin (calbindin 2, Calb2) in the hippocampus of $S O D 1^{G 93 A}$ mice (Chung et al., 2005). In addition, parvalbumin overexpression in $S O D 1^{G 93 A}$ mice resulted in a delayed disease onset and a prolonged survival of these mice (Beers et al., 2001). Since calretinin is a protein of similar function, one could suggest an equal effect in SOD1 disease model mice upon overexpression. Higher $\mathrm{Ca}^{2+}$ buffering capability seems to alter neurotoxic effects, but would not be suspected to completely prevent the disease. Thus, the findings of this study of elevated levels in Calb2 and parvalbumin expression in resistant and relatively 
resistant $\mathrm{MNs}$, may rather implicate a higher tolerance to neurotoxic stress in the BCV MNs than a total resistance (Fig. 34).
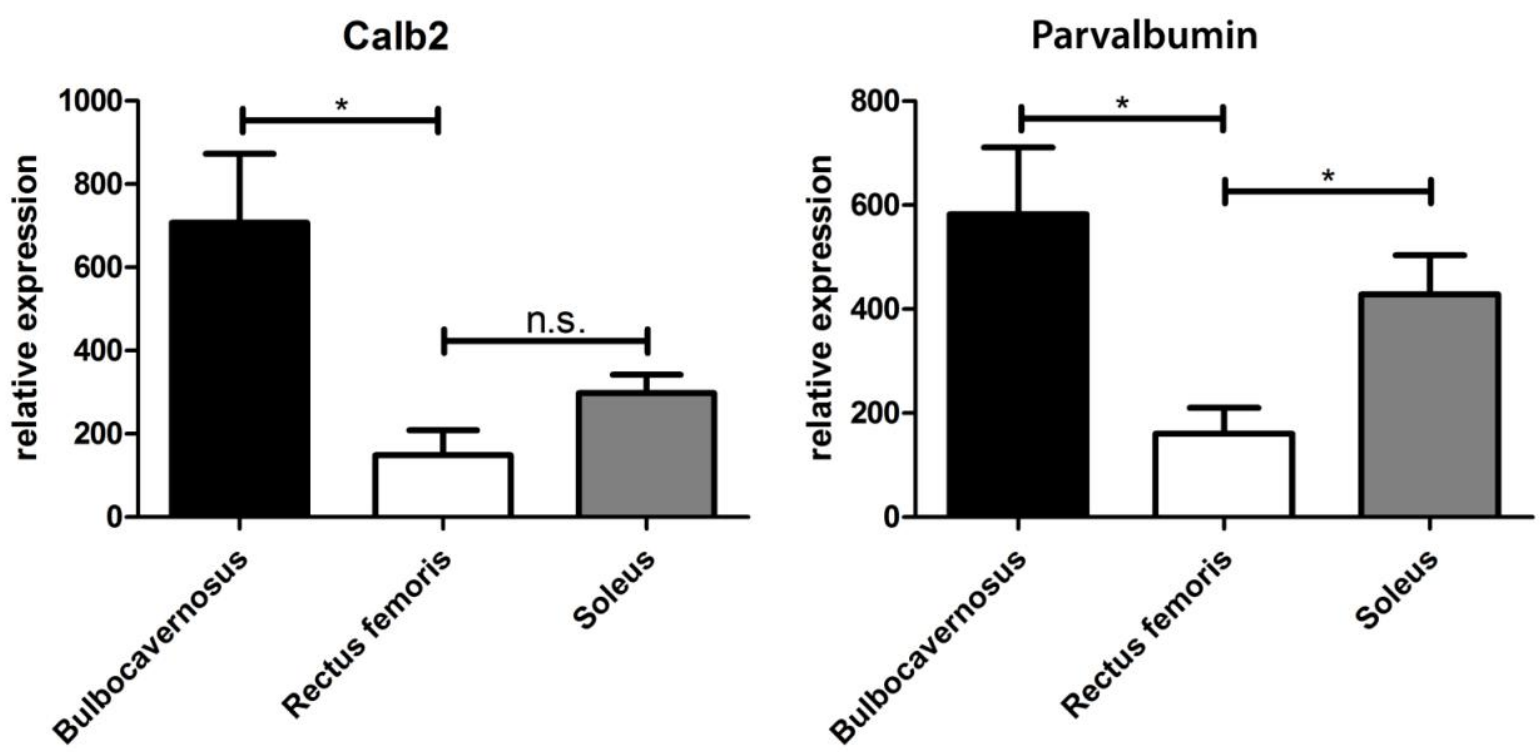

Fig. 34 Relative expression levels of Calb2 and Parvalbumin Array data showing the relative expression levels in $B C V$ and $S$ MNs compared to their expression in $R F M N s(n=4)$. Significant differences are indicated (t-student's test) ${ }^{*} p<0.05,{ }^{* *} p<0.01,{ }^{* * *} p<0.005,{ }^{* * * *} p<0.001$

\subsection{TDP-43 enhancer /suppressor model system}

In this thesis a novel model systems was used to achieve stable MN-specific longterm expression of TDP-43 in chick spinal cord. Through this system it could be shown that TDP-43-mediated MN loss requires a functional RNA-binding motif. Remarkably, these result obtained in the chick precisely mirrored those obtained for TDP-43-mediated toxicity after same site-integrated expression of TDP-43 variants in Drosophila (Voigt et al., 2010). This showed that the fly could be used as a model for TDP-43-mediated toxicity in vertebrate models. The fly system was already under further use in the context of this thesis, where TDP-43 expression in the rough eye screen was utilized in order to investigate the functional properties of possible candidates in neuroprotection. As mentioned before, one of the candidates, Ubxn4, 
showed an enhancement of TDP-43-mediated neurotoxicity upon knock down, consistent with findings indicating a neuroprotective role of Ubxn4 in other studies. The next step will be to perform an overexpression of Ubxn4 in fly and chick system, to test whether this would achieve the opposing impact on TDP-43-mediated toxicity, consistent with a possible role in neuroprotection. Screening of all possible candidates in these model systems may reveal novel mediators of neuroprotection in ALS-like TDP-43 proteinopathy and MN vulnerability in general.

\subsection{Conclusion}

All neuroprotective functions that are discussed for the candidates analysed in this thesis do not seem to mediate the final resistance of MNs towards MNDs. It is possible that the effects of the differentially expressed genes converge to exert protective function in specific MN subtypes. These protective functions are 'shared' for the candidate genes Lifr, Lxn and Ubxn4 in BCV and S MNs. Therefore, Cart is the most prominent candidate gene for mediating resistance towards MNDs, since it was shown to be exclusively expressed in resistant $B C V$ MNs. Functional analysis of Cart in Drosophila rough eye screen and TDP-43 chick model system will reveal its potential neuroprotective role. 


\section{References}

Aagaard, A., Listwan, P., Cowieson, N., Huber, T., Ravasi, T., Wells, C.A., Flanagan, J.U., Kellie, S., Hume, D.A., Kobe, B., and Martin, J.L. (2005). An inflammatory role for the mammalian carboxypeptidase inhibitor latexin: relationship to cystatins and the tumor suppressor TIG1. Structure 13, 309-317.

Almeida, R.D., Manadas, B.J., Melo, C.V., Gomes, J.R., Mendes, C.S., Graos, M.M., Carvalho, R.F., Carvalho, A.P., and Duarte, C.B. (2005). Neuroprotection by BDNF against glutamate-induced apoptotic cell death is mediated by ERK and PI3-kinase pathways. Cell Death Differ 12, 1329-1343.

Atkin, J.D., Farg, M.A., Turner, B.J., Tomas, D., Lysaght, J.A., Nunan, J., Rembach, A., Nagley, P., Beart, P.M., Cheema, S.S., and Horne, M.K. (2006). Induction of the unfolded protein response in familial amyotrophic lateral sclerosis and association of protein-disulfide isomerase with superoxide dismutase 1. J Biol Chem 281, 3015230165.

Baechtold, H., Kuroda, M., Sok, J., Ron, D., Lopez, B.S., and Akhmedov, A.T. (1999). Human $75-\mathrm{kDa}$ DNA-pairing protein is identical to the pro-oncoprotein TLS/FUS and is able to promote D-loop formation. J Biol Chem 274, 34337-34342.

Ballar, P., Shen, Y., Yang, H., and Fang, S. (2006). The role of a novel p97/valosincontaining protein-interacting motif of gp78 in endoplasmic reticulum-associated degradation. J Biol Chem 281, 35359-35368.

Banner, L.R., and Patterson, P.H. (1994). Major changes in the expression of the mRNAs for cholinergic differentiation factor/leukemia inhibitory factor and its receptor after injury to adult peripheral nerves and ganglia. Proc Natl Acad Sci U S A 91, 7109-7113.

Beers, D.R., Henkel, J.S., Xiao, Q., Zhao, W., Wang, J., Yen, A.A., Siklos, L., McKercher, S.R., and Appel, S.H. (2006). Wild-type microglia extend survival in PU.1 knockout mice with familial amyotrophic lateral sclerosis. Proc Natl Acad Sci U S A 103, 16021-16026.

Beers, D.R., Ho, B.K., Siklos, L., Alexianu, M.E., Mosier, D.R., Mohamed, A.H., Otsuka, Y., Kozovska, M.E., McAlhany, R.E., Smith, R.G., and Appel, S.H. (2001). Parvalbumin overexpression alters immune-mediated increases in intracellular calcium, and delays disease onset in a transgenic model of familial amyotrophic lateral sclerosis. J Neurochem 79, 499-509.

Bertrand, P., Akhmedov, A.T., Delacote, F., Durrbach, A., and Lopez, B.S. (1999). Human POMp75 is identified as the pro-oncoprotein TLS/FUS: both POMp75 and POMp100 DNA homologous pairing activities are associated to cell proliferation. Oncogene 18, 4515-4521.

Boillee, S., Vande Velde, C., and Cleveland, D.W. (2006). ALS: a disease of motor neurons and their nonneuronal neighbors. Neuron 52, 39-59. 
Bonetti, B., Pohl, J., Gao, Y.L., and Raine, C.S. (1997). Cell death during autoimmune demyelination: effector but not target cells are eliminated by apoptosis. $J$ Immunol 159, 5733-5741.

Bonetti, B., Stegagno, C., Cannella, B., Rizzuto, N., Moretto, G., and Raine, C.S. (1999). Activation of NF-kappaB and c-jun transcription factors in multiple sclerosis lesions. Implications for oligodendrocyte pathology. Am J Pathol 155, 1433-1438.

Borthwick, G.M., Johnson, M.A., Ince, P.G., Shaw, P.J., and Turnbull, D.M. (1999). Mitochondrial enzyme activity in amyotrophic lateral sclerosis: implications for the role of mitochondria in neuronal cell death. Ann Neurol 46, 787-790.

Bowling, A.C., Schulz, J.B., Brown, R.H., Jr., and Beal, M.F. (1993). Superoxide dismutase activity, oxidative damage, and mitochondrial energy metabolism in familial and sporadic amyotrophic lateral sclerosis. J Neurochem 61, 2322-2325.

Bowman, T., Garcia, R., Turkson, J., and Jove, R. (2000). STATs in oncogenesis. Oncogene 19, 2474-2488.

Brodsky, J.L. (2007). The protective and destructive roles played by molecular chaperones during ERAD (endoplasmic-reticulum-associated degradation). Biochem J 404, 353-363.

Bruijn, L.I., Houseweart, M.K., Kato, S., Anderson, K.L., Anderson, S.D., Ohama, E., Reaume, A.G., Scott, R.W., and Cleveland, D.W. (1998). Aggregation and motor neuron toxicity of an ALS-linked SOD1 mutant independent from wild-type SOD1. Science 281, 1851-1854.

Bruijn, L.I., Miller, T.M., and Cleveland, D.W. (2004). Unraveling the mechanisms involved in motor neuron degeneration in ALS. Annu Rev Neurosci 27, 723-749.

Buratti, E., and Baralle, F.E. (2001). Characterization and functional implications of the RNA binding properties of nuclear factor TDP-43, a novel splicing regulator of CFTR exon 9. J Biol Chem 276, 36337-36343.

Butzkueven, H., Zhang, J.G., Soilu-Hanninen, M., Hochrein, H., Chionh, F., Shipham, K.A., Emery, B., Turnley, A.M., Petratos, S., Ernst, M., et al. (2002). LIF receptor signaling limits immune-mediated demyelination by enhancing oligodendrocyte survival. Nat Med 8, 613-619.

Carriedo, S.G., Sensi, S.L., Yin, H.Z., and Weiss, J.H. (2000). AMPA exposures induce mitochondrial $\mathrm{Ca}(2+)$ overload and $\mathrm{ROS}$ generation in spinal motor neurons in vitro. J Neurosci 20, 240-250.

Cartegni, L., Maconi, M., Morandi, E., Cobianchi, F., Riva, S., and Biamonti, G. (1996). hnRNP A1 selectively interacts through its Gly-rich domain with different RNA-binding proteins. J Mol Biol 259, 337-348.

Cheah, B.C., Vucic, S., Krishnan, A.V., and Kiernan, M.C. (2010). Riluzole, neuroprotection and amyotrophic lateral sclerosis. Curr Med Chem 17, 1942-1199.

Chen, D., Shen, L., Wang, L., Lu, A., Zhang, H., Zhang, X., Zhang, Y., Shui, W., Li, L., Fan, D., and Zhang, J. (2007). Association of polymorphisms in vascular 
endothelial growth factor gene with the age of onset of amyotrophic lateral sclerosis. Amyotroph Lateral Scler 8, 144-149.

Chio, A., Benzi, G., Dossena, M., Mutani, R., and Mora, G. (2005). Severely increased risk of amyotrophic lateral sclerosis among Italian professional football players. Brain 128, 472-476.

Chu, C.T. (2010). Tickled PINK1: mitochondrial homeostasis and autophagy in recessive Parkinsonism. Biochim Biophys Acta 1802, 20-28.

Chung, Y.H., Joo, K.M., Nam, R.H., Cho, M.H., Kim, D.J., Lee, W.B., and Cha, C.I. (2005). Decreased expression of calretinin in the cerebral cortex and hippocampus of SOD1G93A transgenic mice. Brain Res 1035, 105-109.

Crapo, J.D., Oury, T., Rabouille, C., Slot, J.W., and Chang, L.Y. (1992). Copper,zinc superoxide dismutase is primarily a cytosolic protein in human cells. Proc Natl Acad Sci U S A 89, 10405-10409.

Crozat, A., Aman, P., Mandahl, N., and Ron, D. (1993). Fusion of CHOP to a novel RNA-binding protein in human myxoid liposarcoma. Nature 363, 640-644.

Curtis, R., Scherer, S.S., Somogyi, R., Adryan, K.M., Ip, N.Y., Zhu, Y., Lindsay, R.M., and DiStefano, P.S. (1994). Retrograde axonal transport of LIF is increased by peripheral nerve injury: correlation with increased LIF expression in distal nerve. Neuron 12, 191-204.

D'Souza, S.D., Bonetti, B., Balasingam, V., Cashman, N.R., Barker, P.A., Troutt, A.B., Raine, C.S., and Antel, J.P. (1996). Multiple sclerosis: Fas signaling in oligodendrocyte cell death. J Exp Med 184, 2361-2370.

Davis, S., Aldrich, T.H., Stahl, N., Pan, L., Taga, T., Kishimoto, T., Ip, N.Y., and Yancopoulos, G.D. (1993). LIFR beta and gp130 as heterodimerizing signal transducers of the tripartite CNTF receptor. Science 260, 1805-1808.

De Maria, R., Todaro, M., Stassi, G., Di Blasi, F., Giordano, M., Galluzzo, A., and Giordano, C. (1994). Defective T cell receptor/CD3 complex signaling in human type I diabetes. Eur J Immunol 24, 999-1002.

Del Bo, R., Ghezzi, S., Corti, S., Pandolfo, M., Ranieri, M., Santoro, D., Ghione, I., Prelle, A., Orsetti, V., Mancuso, M., et al. (2009). TARDBP (TDP-43) sequence analysis in patients with familial and sporadic ALS: identification of two novel mutations. Eur J Neurol 16, 727-732.

DeLaBarre, B., Christianson, J.C., Kopito, R.R., and Brunger, A.T. (2006). Central pore residues mediate the p97/VCP activity required for ERAD. Mol Cell 22, 451-462.

Deng, H.X., Shi, Y., Furukawa, Y., Zhai, H., Fu, R., Liu, E., Gorrie, G.H., Khan, M.S., Hung, W.Y., Bigio, E.H., et al. (2006). Conversion to the amyotrophic lateral sclerosis phenotype is associated with intermolecular linked insoluble aggregates of SOD1 in mitochondria. Proc Natl Acad Sci U S A 103, 7142-7147. 
Ding, H., Schwarz, D.S., Keene, A., Affar el, B., Fenton, L., Xia, X., Shi, Y., Zamore, P.D., and Xu, Z. (2003). Selective silencing by RNAi of a dominant allele that causes amyotrophic lateral sclerosis. Aging Cell 2, 209-217.

Dion, P.A., Daoud, H., and Rouleau, G.A. (2009). Genetics of motor neuron disorders: new insights into pathogenic mechanisms. Nat Rev Genet 10, 769-782.

Dreveny, I., Kondo, H., Uchiyama, K., Shaw, A., Zhang, X., and Freemont, P.S. (2004). Structural basis of the interaction between the AAA ATPase p97/VCP and its adaptor protein p47. EMBO J 23, 1030-1039.

Dreyfuss, G., Matunis, M.J., Pinol-Roma, S., and Burd, C.G. (1993). hnRNP proteins and the biogenesis of mRNA. Annu Rev Biochem 62, 289-321.

Dubessy, C., Cartier, D., Lectez, B., Bucharles, C., Chartrel, N., Montero-Hadjadje, M., Bizet, P., Chatenet, D., Tostivint, H., Scalbert, E., et al. (2008). Characterization of urotensin II, distribution of urotensin II, urotensin II-related peptide and UT receptor mRNAs in mouse: evidence of urotensin II at the neuromuscular junction. J Neurochem 107, 361-374.

Duennwald, M.L., and Lindquist, S. (2008). Impaired ERAD and ER stress are early and specific events in polyglutamine toxicity. Genes Dev 22, 3308-3319.

Feiguin, F., Godena, V.K., Romano, G., D'Ambrogio, A., Klima, R., and Baralle, F.E. (2009). Depletion of TDP-43 affects Drosophila motoneurons terminal synapsis and locomotive behavior. FEBS Lett 583, 1586-1592.

Ferri, A., Cozzolino, M., Crosio, C., Nencini, M., Casciati, A., Gralla, E.B., Rotilio, G., Valentine, J.S., and Carri, M.T. (2006). Familial ALS-superoxide dismutases associate with mitochondria and shift their redox potentials. Proc Natl Acad Sci U S A $103,13860-13865$.

Forman, M.S., Mackenzie, I.R., Cairns, N.J., Swanson, E., Boyer, P.J., Drachman, D.A., Jhaveri, B.S., Karlawish, J.H., Pestronk, A., Smith, T.W., et al. (2006). Novel ubiquitin neuropathology in frontotemporal dementia with valosin-containing protein gene mutations. J Neuropathol Exp Neurol 65, 571-581.

Frey, D., Schneider, C., Xu, L., Borg, J., Spooren, W., and Caroni, P. (2000). Early and selective loss of neuromuscular synapse subtypes with low sprouting competence in motoneuron diseases. J Neurosci 20, 2534-2542.

Friedlander, R.M., Brown, R.H., Gagliardini, V., Wang, J., and Yuan, J. (1997). Inhibition of ICE slows ALS in mice. Nature 388, 31.

Fukunaga, K., and Miyamoto, E. (1998). Role of MAP kinase in neurons. Mol Neurobiol 16, 79-95.

Garcia-Castellanos, R., Bonet-Figueredo, R., Pallares, I., Ventura, S., Aviles, F.X., Vendrell, J., and Gomis-Rutha, F.X. (2005). Detailed molecular comparison between the inhibition mode of A/B-type carboxypeptidases in the zymogen state and by the endogenous inhibitor latexin. Cell Mol Life Sci 62, 1996-2014. 
Gearing, D.P., Thut, C.J., VandeBos, T., Gimpel, S.D., Delaney, P.B., King, J., Price, V., Cosman, D., and Beckmann, M.P. (1991). Leukemia inhibitory factor receptor is structurally related to the IL-6 signal transducer, gp130. EMBO J 10, 2839-2848.

Gitcho, M.A., Baloh, R.H., Chakraverty, S., Mayo, K., Norton, J.B., Levitch, D., Hatanpaa, K.J., White, C.L., 3rd, Bigio, E.H., Caselli, R., et al. (2008). TDP-43 A315T mutation in familial motor neuron disease. Ann Neurol 63, 535-538.

Gitcho, M.A., Bigio, E.H., Mishra, M., Johnson, N., Weintraub, S., Mesulam, M., Rademakers, R., Chakraverty, S., Cruchaga, C., Morris, J.C., et al. (2009). TARDBP 3 '-UTR variant in autopsy-confirmed frontotemporal lobar degeneration with TDP-43 proteinopathy. Acta Neuropathol 118, 633-645.

Gong, Y.H., Parsadanian, A.S., Andreeva, A., Snider, W.D., and Elliott, J.L. (2000). Restricted expression of $\mathrm{G} 86 \mathrm{R} \mathrm{Cu/Zn}$ superoxide dismutase in astrocytes results in astrocytosis but does not cause motoneuron degeneration. J Neurosci 20, 660-665.

Greenway, M.J., Andersen, P.M., Russ, C., Ennis, S., Cashman, S., Donaghy, C., Patterson, V., Swingler, R., Kieran, D., Prehn, J., et al. (2006). ANG mutations segregate with familial and 'sporadic' amyotrophic lateral sclerosis. Nat Genet 38, 411-413.

Gros-Louis, F., Gaspar, C., and Rouleau, G.A. (2006). Genetics of familial and sporadic amyotrophic lateral sclerosis. Biochim Biophys Acta 1762, 956-972.

Guo, H., Lai, L., Butchbach, M.E., Stockinger, M.P., Shan, X., Bishop, G.A., and Lin, C.L. (2003). Increased expression of the glial glutamate transporter EAAT2 modulates excitotoxicity and delays the onset but not the outcome of ALS in mice. Hum Mol Genet 12, 2519-2532.

Hadano, S., Hand, C.K., Osuga, H., Yanagisawa, Y., Otomo, A., Devon, R.S., Miyamoto, N., Showguchi-Miyata, J., Okada, Y., Singaraja, R., et al. (2001). A gene encoding a putative GTPase regulator is mutated in familial amyotrophic lateral sclerosis 2. Nat Genet 29, 166-173.

Hamson, D.K., Hu, J.H., Krieger, C., and Watson, N.V. (2002). Lumbar motoneuron fate in a mouse model of amyotrophic lateral sclerosis. Neuroreport 13, 2291-2294.

Higgins, C.M., Jung, C., Ding, H., and Xu, Z. (2002). Mutant Cu, Zn superoxide dismutase that causes motoneuron degeneration is present in mitochondria in the CNS. J Neurosci 22, RC215.

Hirsch, C., Gauss, R., Horn, S.C., Neuber, O., and Sommer, T. (2009). The ubiquitylation machinery of the endoplasmic reticulum. Nature 458, 453-460.

Hoess, R., Abremski, K., and Sternberg, N. (1984). The nature of the interaction of the P1 recombinase Cre with the recombining site loxP. Cold Spring Harb Symp Quant Biol 49, 761-768.

Igaz, L.M., Kwong, L.K., Lee, E.B., Chen-Plotkin, A., Swanson, E., Unger, T., Malunda, J., Xu, Y., Winton, M.J., Trojanowski, J.Q., and Lee, V.M. (2011). Dysregulation of the ALS-associated gene TDP-43 leads to neuronal death and degeneration in mice. J Clin Invest 121, 726-738. 
Ince, P., Stout, N., Shaw, P., Slade, J., Hunziker, W., Heizmann, C.W., and Baimbridge, K.G. (1993). Parvalbumin and calbindin D-28k in the human motor system and in motor neuron disease. Neuropathol Appl Neurobiol 19, 291-299.

Jaarsma, D., Haasdijk, E.D., Grashorn, J.A., Hawkins, R., van Duijn, W., Verspaget, H.W., London, J., and Holstege, J.C. (2000). Human Cu/Zn superoxide dismutase (SOD1) overexpression in mice causes mitochondrial vacuolization, axonal degeneration, and premature motoneuron death and accelerates motoneuron disease in mice expressing a familial amyotrophic lateral sclerosis mutant SOD1. Neurobiol Dis 7, 623-643.

Jaiswal, M.K., and Keller, B.U. (2009). Cu/Zn superoxide dismutase typical for familial amyotrophic lateral sclerosis increases the vulnerability of mitochondria and perturbs Ca2+ homeostasis in SOD1G93A mice. Mol Pharmacol 75, 478-489.

Jaiswal, M.K., Zech, W.D., Goos, M., Leutbecher, C., Ferri, A., Zippelius, A., Carri, M.T., Nau, R., and Keller, B.U. (2009). Impairment of mitochondrial calcium handling in a mtSOD1 cell culture model of motoneuron disease. BMC Neurosci 10, 64.

Jiang, Z., Michal, J.J., Tobey, D.J., Wang, Z., Macneil, M.D., and Magnuson, N.S. (2008). Comparative understanding of UTS2 and UTS2R genes for their involvement in type 2 diabetes mellitus. Int J Biol Sci 4, 96-102.

Johnson, B.S., Snead, D., Lee, J.J., McCaffery, J.M., Shorter, J., and Gitler, A.D. (2009). TDP-43 is intrinsically aggregation-prone, and amyotrophic lateral sclerosislinked mutations accelerate aggregation and increase toxicity. J Biol Chem 284, 20329-20339.

Johnson, J.O., Mandrioli, J., Benatar, M., Abramzon, Y., Van Deerlin, V.M., Trojanowski, J.Q., Gibbs, J.R., Brunetti, M., Gronka, S., Wuu, J., et al. (2010). Exome sequencing reveals VCP mutations as a cause of familial ALS. Neuron 68, 857-864.

Jung, C., Higgins, C.M., and Xu, Z. (2002). Mitochondrial electron transport chain complex dysfunction in a transgenic mouse model for amyotrophic lateral sclerosis. $J$ Neurochem 83, 535-545.

Kabashi, E., Valdmanis, P.N., Dion, P., Spiegelman, D., McConkey, B.J., Vande Velde, C., Bouchard, J.P., Lacomblez, L., Pochigaeva, K., Salachas, F., et al. (2008). TARDBP mutations in individuals with sporadic and familial amyotrophic lateral sclerosis. Nat Genet 40, 572-574.

Kaeser, S.A., Herzig, M.C., Coomaraswamy, J., Kilger, E., Selenica, M.L., Winkler, D.T., Staufenbiel, M., Levy, E., Grubb, A., and Jucker, M. (2007). Cystatin C modulates cerebral beta-amyloidosis. Nat Genet 39, 1437-1439.

Kanehara, K., Kawaguchi, S., and Ng, D.T. (2007). The EDEM and Yos9p families of lectin-like ERAD factors. Semin Cell Dev Biol 18, 743-750.

Kawakami, K. (2007). Tol2: a versatile gene transfer vector in vertebrates. Genome Biol 8 Suppl 1, S7. 
Kawakami, K., and Noda, T. (2004). Transposition of the Tol2 element, an Ac-like element from the Japanese medaka fish Oryzias latipes, in mouse embryonic stem cells. Genetics 166, 895-899.

Kawakami, K., and Shima, A. (1999). Identification of the Tol2 transposase of the medaka fish Oryzias latipes that catalyzes excision of a nonautonomous Tol2 element in zebrafish Danio rerio. Gene 240, 239-244.

Kawamata, H., and Manfredi, G. (2008). Different regulation of wild-type and mutant $\mathrm{Cu}, \mathrm{Zn}$ superoxide dismutase localization in mammalian mitochondria. Hum Mol Genet 17, 3303-3317.

Kikuchi, H., Almer, G., Yamashita, S., Guegan, C., Nagai, M., Xu, Z., Sosunov, A.A., McKhann, G.M., 2nd, and Przedborski, S. (2006). Spinal cord endoplasmic reticulum stress associated with a microsomal accumulation of mutant superoxide dismutase-1 in an ALS model. Proc Natl Acad Sci U S A 103, 6025-6030.

Kirkinezos, I.G., Bacman, S.R., Hernandez, D., Oca-Cossio, J., Arias, L.J., PerezPinzon, M.A., Bradley, W.G., and Moraes, C.T. (2005). Cytochrome c association with the inner mitochondrial membrane is impaired in the CNS of G93A-SOD1 mice. J Neurosci 25, 164-172.

Klivenyi, P., Ferrante, R.J., Matthews, R.T., Bogdanov, M.B., Klein, A.M., Andreassen, O.A., Mueller, G., Wermer, M., Kaddurah-Daouk, R., and Beal, M.F. (1999). Neuroprotective effects of creatine in a transgenic animal model of amyotrophic lateral sclerosis. Nat Med 5, 347-350.

Kong, J., and Xu, Z. (1998). Massive mitochondrial degeneration in motor neurons triggers the onset of amyotrophic lateral sclerosis in mice expressing a mutant SOD1. J Neurosci 18, 3241-3250.

Kostic, V., Jackson-Lewis, V., de Bilbao, F., Dubois-Dauphin, M., and Przedborski, S. (1997). Bcl-2: prolonging life in a transgenic mouse model of familial amyotrophic lateral sclerosis. Science 277, 559-562.

Kostova, Z., Tsai, Y.C., and Weissman, A.M. (2007). Ubiquitin ligases, critical mediators of endoplasmic reticulum-associated degradation. Semin Cell Dev Biol 18, 770-779.

Kwiatkowski, T.J., Jr., Bosco, D.A., Leclerc, A.L., Tamrazian, E., Vanderburg, C.R., Russ, C., Davis, A., Gilchrist, J., Kasarskis, E.J., Munsat, T., et al. (2009). Mutations in the FUS/TLS gene on chromosome 16 cause familial amyotrophic lateral sclerosis. Science 323, 1205-1208.

Kwong, L.K., Neumann, M., Sampathu, D.M., Lee, V.M., and Trojanowski, J.Q. (2007). TDP-43 proteinopathy: the neuropathology underlying major forms of sporadic and familial frontotemporal lobar degeneration and motor neuron disease. Acta Neuropathol 114, 63-70.

Langham, R.G., Kelly, D.J., Gow, R.M., Zhang, Y., Dowling, J.K., Thomson, N.M., and Gilbert, R.E. (2004). Increased expression of urotensin II and urotensin II receptor in human diabetic nephropathy. Am J Kidney Dis 44, 826-831. 
Lavara-Culebras, E., and Paricio, N. (2007). Drosophila DJ-1 mutants are sensitive to oxidative stress and show reduced lifespan and motor deficits. Gene 400, 158-165.

Lee, S.K., Jurata, L.W., Funahashi, J., Ruiz, E.C., and Pfaff, S.L. (2004). Analysis of embryonic motoneuron gene regulation: derepression of general activators function in concert with enhancer factors. Development 131, 3295-3306.

Li, M., Ona, V.O., Guegan, C., Chen, M., Jackson-Lewis, V., Andrews, L.J., Olszewski, A.J., Stieg, P.E., Lee, J.P., Przedborski, S., and Friedlander, R.M. (2000). Functional role of caspase-1 and caspase-3 in an ALS transgenic mouse model. Science 288, 335-339.

Li, M., Sendtner, M., and Smith, A. (1995). Essential function of LIF receptor in motor neurons. Nature 378, 724-727.

Li, Y., Ray, P., Rao, E.J., Shi, C., Guo, W., Chen, X., Woodruff, E.A., 3rd, Fushimi, K., and Wu, J.Y. (2010). A Drosophila model for TDP-43 proteinopathy. Proc Natl Acad Sci U S A 107, 3169-3174.

Liang, J., Yin, C., Doong, H., Fang, S., Peterhoff, C., Nixon, R.A., and Monteiro, M.J. (2006). Characterization of erasin (UBXD2): a new ER protein that promotes ERassociated protein degradation. J Cell Sci 119, 4011-4024.

Liang, Y., and Van Zant, G. (2008). Aging stem cells, latexin, and longevity. Exp Cell Res 314, 1962-1972.

Lino, M.M., Schneider, C., and Caroni, P. (2002). Accumulation of SOD1 mutants in postnatal motoneurons does not cause motoneuron pathology or motoneuron disease. J Neurosci 22, 4825-4832.

Livingstone, M., Ruan, H., Weiner, J., Clauser, K.R., Strack, P., Jin, S., Williams, A., Greulich, H., Gardner, J., Venere, M., et al. (2005). Valosin-containing protein phosphorylation at Ser784 in response to DNA damage. Cancer Res 65, 7533-7540.

Lucchinetti, C., Bruck, W., Parisi, J., Scheithauer, B., Rodriguez, M., and Lassmann, H. (2000). Heterogeneity of multiple sclerosis lesions: implications for the pathogenesis of demyelination. Ann Neurol 47, 707-717.

Manfredi, G., and Xu, Z. (2005). Mitochondrial dysfunction and its role in motor neuron degeneration in ALS. Mitochondrion 5, 77-87.

Mao, P., Ardeshiri, A., Jacks, R., Yang, S., Hurn, P.D., and Alkayed, N.J. (2007). Mitochondrial mechanism of neuroprotection by CART. Eur J Neurosci 26, 624-632.

Matsuda, T., and Cepko, C.L. (2004). Electroporation and RNA interference in the rodent retina in vivo and in vitro. Proc Natl Acad Sci U S A 101, 16-22.

Matunis, E.L., Matunis, M.J., and Dreyfuss, G. (1992). Characterization of the major hnRNP proteins from Drosophila melanogaster. J Cell Biol 116, 257-269.

Mayeda, A., Munroe, S.H., Caceres, J.F., and Krainer, A.R. (1994). Function of conserved domains of hnRNP A1 and other hnRNP A/B proteins. EMBO J 13, 54835495. 
McCracken, A.A., and Brodsky, J.L. (1996). Assembly of ER-associated protein degradation in vitro: dependence on cytosol, calnexin, and ATP. J Cell Biol 132, 291298.

Mi, W., Pawlik, M., Sastre, M., Jung, S.S., Radvinsky, D.S., Klein, A.M., Sommer, J., Schmidt, S.D., Nixon, R.A., Mathews, P.M., and Levy, E. (2007). Cystatin C inhibits amyloid-beta deposition in Alzheimer's disease mouse models. Nat Genet 39, 14401442.

Middleton, G., Hamanoue, M., Enokido, Y., Wyatt, S., Pennica, D., Jaffray, E., Hay, R.T., and Davies, A.M. (2000). Cytokine-induced nuclear factor kappa B activation promotes the survival of developing neurons. J Cell Biol 148, 325-332.

Miguel, L., Frebourg, T., Campion, D., and Lecourtois, M. (2011). Both cytoplasmic and nuclear accumulations of the protein are neurotoxic in Drosophila models of TDP-43 proteinopathies. Neurobiol Dis 41, 398-406.

Mirnics, Z.K., Mirnics, K., Terrano, D., Lewis, D.A., Sisodia, S.S., and Schor, N.F. (2003). DNA microarray profiling of developing PS1-deficient mouse brain reveals complex and coregulated expression changes. Mol Psychiatry 8, 863-878.

Mitsumoto, H.C., D.A. and Pioro, E.P. (1998). Amyotrophic Lateral Sclerosis (Davis, Philadelphia ).

Morita, Y., Naka, T., Kawazoe, Y., Fujimoto, M., Narazaki, M., Nakagawa, R., Fukuyama, H., Nagata, S., and Kishimoto, T. (2000). Signals transducers and activators of transcription (STAT)-induced STAT inhibitor-1 (SSI-1)/suppressor of cytokine signaling-1 (SOCS-1) suppresses tumor necrosis factor alpha-induced cell death in fibroblasts. Proc Natl Acad Sci U S A 97, 5405-5410.

Mulder, D.W. (1982). Clinical limits of amyotrophic lateral sclerosis. Adv Neurol 36, 15-22.

Munch, C., Sedlmeier, R., Meyer, T., Homberg, V., Sperfeld, A.D., Kurt, A., Prudlo, J., Peraus, G., Hanemann, C.O., Stumm, G., and Ludolph, A.C. (2004). Point mutations of the p150 subunit of dynactin (DCTN1) gene in ALS. Neurology 63, 724726.

Neumann, M., Sampathu, D.M., Kwong, L.K., Truax, A.C., Micsenyi, M.C., Chou, T.T., Bruce, J., Schuck, T., Grossman, M., Clark, C.M., et al. (2006). Ubiquitinated TDP-43 in frontotemporal lobar degeneration and amyotrophic lateral sclerosis. Science 314, 130-133.

Nishimura, A.L., Mitne-Neto, M., Silva, H.C., Richieri-Costa, A., Middleton, S., Cascio, D., Kok, F., Oliveira, J.R., Gillingwater, T., Webb, J., et al. (2004). A mutation in the vesicle-trafficking protein VAPB causes late-onset spinal muscular atrophy and amyotrophic lateral sclerosis. Am J Hum Genet 75, 822-831.

Nishitoh, H., Kadowaki, H., Nagai, A., Maruyama, T., Yokota, T., Fukutomi, H., Noguchi, T., Matsuzawa, A., Takeda, K., and Ichijo, H. (2008). ALS-linked mutant SOD1 induces ER stress- and ASK1-dependent motor neuron death by targeting Derlin-1. Genes Dev 22, 1451-1464. 
Normant, E., Martres, M.P., Schwartz, J.C., and Gros, C. (1995). Purification, cDNA cloning, functional expression, and characterization of a $26-\mathrm{kDa}$ endogenous mammalian carboxypeptidase inhibitor. Proc Natl Acad Sci U S A 92, 12225-12229.

Nowis, D., McConnell, E., and Wojcik, C. (2006). Destabilization of the VCP-Ufd1Npl4 complex is associated with decreased levels of ERAD substrates. Exp Cell Res 312, 2921-2932.

Okado-Matsumoto, A., and Fridovich, I. (2001). Subcellular distribution of superoxide dismutases (SOD) in rat liver: $\mathrm{Cu}, \mathrm{Zn}-\mathrm{SOD}$ in mitochondria. J Biol Chem 276, 3838838393.

Pasinelli, P., Belford, M.E., Lennon, N., Bacskai, B.J., Hyman, B.T., Trotti, D., and Brown, R.H., Jr. (2004). Amyotrophic lateral sclerosis-associated SOD1 mutant proteins bind and aggregate with Bcl-2 in spinal cord mitochondria. Neuron 43, 1930 .

Pasinelli, P., Borchelt, D.R., Houseweart, M.K., Cleveland, D.W., and Brown, R.H., Jr. (1998). Caspase-1 is activated in neural cells and tissue with amyotrophic lateral sclerosis-associated mutations in copper-zinc superoxide dismutase. Proc Natl Acad Sci U S A 95, 15763-15768.

Pasinelli, P., and Brown, R.H. (2006). Molecular biology of amyotrophic lateral sclerosis: insights from genetics. Nat Rev Neurosci 7, 710-723.

Pelletier, G., Lihrmann, I., and Vaudry, H. (2002). Role of androgens in the regulation of urotensin II precursor mRNA expression in the rat brainstem and spinal cord. Neuroscience 115, 525-532.

Pesiridis, G.S., Lee, V.M., and Trojanowski, J.Q. (2009). Mutations in TDP-43 link glycine-rich domain functions to amyotrophic lateral sclerosis. Hum Mol Genet 18, R156-162.

Pramatarova, A., Laganiere, J., Roussel, J., Brisebois, K., and Rouleau, G.A. (2001). Neuron-specific expression of mutant superoxide dismutase 1 in transgenic mice does not lead to motor impairment. J Neurosci 21, 3369-3374.

Pun, S., Santos, A.F., Saxena, S., Xu, L., and Caroni, P. (2006). Selective vulnerability and pruning of phasic motoneuron axons in motoneuron disease alleviated by CNTF. Nat Neurosci 9, 408-419.

Pye, V.E., Beuron, F., Keetch, C.A., McKeown, C., Robinson, C.V., Meyer, H.H., Zhang, X., and Freemont, P.S. (2007). Structural insights into the p97-Ufd1-Npl4 complex. Proc Natl Acad Sci U S A 104, 467-472.

Rabizadeh, S., Gralla, E.B., Borchelt, D.R., Gwinn, R., Valentine, J.S., Sisodia, S., Wong, P., Lee, M., Hahn, H., and Bredesen, D.E. (1995). Mutations associated with amyotrophic lateral sclerosis convert superoxide dismutase from an antiapoptotic gene to a proapoptotic gene: studies in yeast and neural cells. Proc Natl Acad Sci U S A 92, 3024-3028. 
Rensink, A.A., Verbeek, M.M., Otte-Holler, I., ten Donkelaar, H.T., de Waal, R.M., and Kremer, B. (2002). Inhibition of amyloid-beta-induced cell death in human brain pericytes in vitro. Brain Res 952, 111-121.

Richly, H., Rape, M., Braun, S., Rumpf, S., Hoege, C., and Jentsch, S. (2005). A series of ubiquitin binding factors connects CDC48/p97 to substrate multiubiquitylation and proteasomal targeting. Cell 120, 73-84.

Ritson, G.P., Custer, S.K., Freibaum, B.D., Guinto, J.B., Geffel, D., Moore, J., Tang, W., Winton, M.J., Neumann, M., Trojanowski, J.Q., et al. (2010). TDP-43 mediates degeneration in a novel Drosophila model of disease caused by mutations in VCP/p97. J Neurosci 30, 7729-7739.

Rosen, D.R. (1993). Mutations in Cu/Zn superoxide dismutase gene are associated with familial amyotrophic lateral sclerosis. Nature 364, 362.

Rothstein, J.D., Dykes-Hoberg, M., Pardo, C.A., Bristol, L.A., Jin, L., Kuncl, R.W., Kanai, Y., Hediger, M.A., Wang, Y., Schielke, J.P., and Welty, D.F. (1996). Knockout of glutamate transporters reveals a major role for astroglial transport in excitotoxicity and clearance of glutamate. Neuron 16, 675-686.

Rothstein, J.D., Jin, L., Dykes-Hoberg, M., and Kuncl, R.W. (1993). Chronic inhibition of glutamate uptake produces a model of slow neurotoxicity. Proc Natl Acad Sci U S A 90, 6591-6595.

Rothstein, J.D., and Kuncl, R.W. (1995). Neuroprotective strategies in a model of chronic glutamate-mediated motor neuron toxicity. J Neurochem 65, 643-651.

Rothstein, J.D., Martin, L.J., and Kuncl, R.W. (1992). Decreased glutamate transport by the brain and spinal cord in amyotrophic lateral sclerosis. $\mathrm{N}$ Engl $\mathrm{J}$ Med 326, 1464-1468.

Rothstein, J.D., Tsai, G., Kuncl, R.W., Clawson, L., Cornblath, D.R., Drachman, D.B., Pestronk, A., Stauch, B.L., and Coyle, J.T. (1990). Abnormal excitatory amino acid metabolism in amyotrophic lateral sclerosis. Ann Neurol 28, 18-25.

Rutherford, N.J., Zhang, Y.J., Baker, M., Gass, J.M., Finch, N.A., Xu, Y.F., Stewart, H., Kelley, B.J., Kuntz, K., Crook, R.J., et al. (2008). Novel mutations in TARDBP (TDP-43) in patients with familial amyotrophic lateral sclerosis. PLoS Genet 4, e1000193.

Sato, Y., Kasai, T., Nakagawa, S., Tanabe, K., Watanabe, T., Kawakami, K., and Takahashi, Y. (2007). Stable integration and conditional expression of electroporated transgenes in chicken embryos. Dev Biol 305, 616-624.

Saxena, S., Cabuy, E., and Caroni, P. (2009). A role for motoneuron subtypeselective ER stress in disease manifestations of FALS mice. Nat Neurosci 12, 627636.

Schaefer, A.M., Sanes, J.R., and Lichtman, J.W. (2005). A compensatory subpopulation of motor neurons in a mouse model of amyotrophic lateral sclerosis. $J$ Comp Neurol 490, 209-219. 
Schmitz, A., and Herzog, V. (2004). Endoplasmic reticulum-associated degradation: exceptions to the rule. Eur $\mathrm{J}$ Cell Biol 83, 501-509.

Schroder, M., and Kaufman, R.J. (2005). The mammalian unfolded protein response. Annu Rev Biochem 74, 739-789.

Segal, R.A., and Greenberg, M.E. (1996). Intracellular signaling pathways activated by neurotrophic factors. Annu Rev Neurosci 19, 463-489.

Sendtner, M., Gotz, R., Holtmann, B., Escary, J.L., Masu, Y., Carroll, P., Wolf, E., Brem, G., Brulet, P., and Thoenen, H. (1996). Cryptic physiological trophic support of motoneurons by LIF revealed by double gene targeting of CNTF and LIF. Curr Biol 6 , 686-694.

Shaner, N.C., Campbell, R.E., Steinbach, P.A., Giepmans, B.N., Palmer, A.E., and Tsien, R.Y. (2004). Improved monomeric red, orange and yellow fluorescent proteins derived from Discosoma sp. red fluorescent protein. Nat Biotechnol 22, 1567-1572.

Shaw, C.E. (2010). Capturing VCP: another molecular piece in the ALS jigsaw puzzle. Neuron 68, 812-814.

Shaw, C.E., Arechavala-Gomeza, V., and Al-Chalabi, A. (2007). Chapter 14 Familial amyotrophic lateral sclerosis. Handb Clin Neurol 82, 279-300.

Shaw, P.J. (2005). Molecular and cellular pathways of neurodegeneration in motor neurone disease. J Neurol Neurosurg Psychiatry 76, 1046-1057.

Siomi, H., and Dreyfuss, G. (1995). A nuclear localization domain in the hnRNP A1 protein. J Cell Biol 129, 551-560.

Soilu-Hanninen, M., Broberg, E., Roytta, M., Mattila, P., Rinne, J., and Hukkanen, V. Expression of LIF and LIF receptor beta in Alzheimer's and Parkinson's diseases. Acta Neurol Scand 121, 44-50.

Sreedharan, J., Blair, I.P., Tripathi, V.B., Hu, X., Vance, C., Rogelj, B., Ackerley, S., Durnall, J.C., Williams, K.L., Buratti, E., et al. (2008). TDP-43 mutations in familial and sporadic amyotrophic lateral sclerosis. Science 319, 1668-1672.

Sturtz, L.A., Diekert, K., Jensen, L.T., Lill, R., and Culotta, V.C. (2001). A fraction of yeast $\mathrm{Cu}, \mathrm{Zn}$-superoxide dismutase and its metallochaperone, CCS, localize to the intermembrane space of mitochondria. A physiological role for SOD1 in guarding against mitochondrial oxidative damage. J Biol Chem 276, 38084-38089.

Subramaniam, J.R., Lyons, W.E., Liu, J., Bartnikas, T.B., Rothstein, J., Price, D.L., Cleveland, D.W., Gitlin, J.D., and Wong, P.C. (2002). Mutant SOD1 causes motor neuron disease independent of copper chaperone-mediated copper loading. Nat Neurosci 5, 301-307.

Swanson, R.A., Liu, J., Miller, J.W., Rothstein, J.D., Farrell, K., Stein, B.A., and Longuemare, M.C. (1997). Neuronal regulation of glutamate transporter subtype expression in astrocytes. J Neurosci 17, 932-940. 
Takahashi, Y., Watanabe, T., Nakagawa, S., Kawakami, K., and Sato, Y. (2008). Transposon-mediated stable integration and tetracycline-inducible expression of electroporated transgenes in chicken embryos. Methods Cell Biol 87, 271-280.

Takeuchi, H., Kobayashi, Y., Ishigaki, S., Doyu, M., and Sobue, G. (2002). Mitochondrial localization of mutant superoxide dismutase 1 triggers caspasedependent cell death in a cellular model of familial amyotrophic lateral sclerosis. $J$ Biol Chem 277, 50966-50972.

Tatom, J.B., Wang, D.B., Dayton, R.D., Skalli, O., Hutton, M.L., Dickson, D.W., and Klein, R.L. (2009). Mimicking aspects of frontotemporal lobar degeneration and Lou Gehrig's disease in rats via TDP-43 overexpression. Mol Ther 17, 607-613.

Trotti, D., Danbolt, N.C., and Volterra, A. (1998). Glutamate transporters are oxidantvulnerable: a molecular link between oxidative and excitotoxic neurodegeneration? Trends Pharmacol Sci 19, 328-334.

Turnley, A.M., and Bartlett, P.F. (2000). Cytokines that signal through the leukemia inhibitory factor receptor-beta complex in the nervous system. J Neurochem 74, 889899.

Urasaki, A., Morvan, G., and Kawakami, K. (2006). Functional dissection of the Tol2 transposable element identified the minimal cis-sequence and a highly repetitive sequence in the subterminal region essential for transposition. Genetics 174, 639649.

Van Damme, P., Van Den Bosch, L., Van Houtte, E., Callewaert, G., and Robberecht, W. (2002). GluR2-dependent properties of AMPA receptors determine the selective vulnerability of motor neurons to excitotoxicity. J Neurophysiol 88,1279 1287.

Vance, C., Rogelj, B., Hortobagyi, T., De Vos, K.J., Nishimura, A.L., Sreedharan, J., Hu, X., Smith, B., Ruddy, D., Wright, P., et al. (2009). Mutations in FUS, an RNA processing protein, cause familial amyotrophic lateral sclerosis type 6 . Science 323 , 1208-1211.

Vande Velde, C., Miller, T.M., Cashman, N.R., and Cleveland, D.W. (2008). Selective association of misfolded ALS-linked mutant SOD1 with the cytoplasmic face of mitochondria. Proc Natl Acad Sci U S A 105, 4022-4027.

Vandermoere, F., El Yazidi-Belkoura, I., Slomianny, C., Demont, Y., Bidaux, G., Adriaenssens, E., Lemoine, J., and Hondermarck, H. (2006). The valosin-containing protein (VCP) is a target of Akt signaling required for cell survival. J Biol Chem 281 , 14307-14313.

Vartanian, T., Li, Y., Zhao, M., and Stefansson, K. (1995). Interferon-gamma-induced oligodendrocyte cell death: implications for the pathogenesis of multiple sclerosis. Mol Med 1, 732-743.

Vembar, S.S., and Brodsky, J.L. (2008). One step at a time: endoplasmic reticulumassociated degradation. Nat Rev Mol Cell Biol 9, 944-957. 
Vijayvergiya, C., Beal, M.F., Buck, J., and Manfredi, G. (2005). Mutant superoxide dismutase 1 forms aggregates in the brain mitochondrial matrix of amyotrophic lateral sclerosis mice. J Neurosci 25, 2463-2470.

Voigt, A., Herholz, D., Fiesel, F.C., Kaur, K., Muller, D., Karsten, P., Weber, S.S., Kahle, P.J., Marquardt, T., and Schulz, J.B. (2010). TDP-43-mediated neuron loss in vivo requires RNA-binding activity. PLoS One 5, e12247.

Volterra, A., Trotti, D., Floridi, S., and Racagni, G. (1994a). Reactive oxygen species inhibit high-affinity glutamate uptake: molecular mechanism and neuropathological implications. Ann N Y Acad Sci 738, 153-162.

Volterra, A., Trotti, D., and Racagni, G. (1994b). Glutamate uptake is inhibited by arachidonic acid and oxygen radicals via two distinct and additive mechanisms. Mol Pharmacol 46, 986-992.

Volterra, A., Trotti, D., Tromba, C., Floridi, S., and Racagni, G. (1994c). Glutamate uptake inhibition by oxygen free radicals in rat cortical astrocytes. J Neurosci 14 , 2924-2932.

Wang, H.Y., Wang, I.F., Bose, J., and Shen, C.K. (2004). Structural diversity and functional implications of the eukaryotic TDP gene family. Genomics 83, 130-139.

Wegorzewska, I., Bell, S., Cairns, N.J., Miller, T.M., and Baloh, R.H. (2009). TDP-43 mutant transgenic mice develop features of ALS and frontotemporal lobar degeneration. Proc Natl Acad Sci U S A 106, 18809-18814.

Weighardt, F., Biamonti, G., and Riva, S. (1995). Nucleo-cytoplasmic distribution of human hnRNP proteins: a search for the targeting domains in hnRNP A1. J Cell Sci 108 ( Pt 2), 545-555.

Wojcik, C., Rowicka, M., Kudlicki, A., Nowis, D., McConnell, E., Kujawa, M., and DeMartino, G.N. (2006). Valosin-containing protein (p97) is a regulator of endoplasmic reticulum stress and of the degradation of $\mathrm{N}$-end rule and ubiquitinfusion degradation pathway substrates in mammalian cells. Mol Biol Cell 17, 46064618.

Wong, M., and Martin, L.J. (2010). Skeletal muscle-restricted expression of human SOD1 causes motor neuron degeneration in transgenic mice. Hum Mol Genet 19, 2284-2302.

Wong, P.C., Pardo, C.A., Borchelt, D.R., Lee, M.K., Copeland, N.G., Jenkins, N.A., Sisodia, S.S., Cleveland, D.W., and Price, D.L. (1995). An adverse property of a familial ALS-linked SOD1 mutation causes motor neuron disease characterized by vacuolar degeneration of mitochondria. Neuron 14, 1105-1116.

Yang, L., Embree, L.J., Tsai, S., and Hickstein, D.D. (1998). Oncoprotein TLS interacts with serine-arginine proteins involved in RNA splicing. J Biol Chem 273, 27761-27764.

Yang, Y., Hentati, A., Deng, H.X., Dabbagh, O., Sasaki, T., Hirano, M., Hung, W.Y., Ouahchi, K., Yan, J., Azim, A.C., et al. (2001). The gene encoding alsin, a protein 
with three guanine-nucleotide exchange factor domains, is mutated in a form of recessive amyotrophic lateral sclerosis. Nat Genet 29, 160-165.

Yokoseki, A., Shiga, A., Tan, C.F., Tagawa, A., Kaneko, H., Koyama, A., Eguchi, H., Tsujino, A., Ikeuchi, T., Kakita, A., et al. (2008). TDP-43 mutation in familial amyotrophic lateral sclerosis. Ann Neurol 63, 538-542.

Yoshida, S., Mulder, D.W., Kurland, L.T., Chu, C.P., and Okazaki, H. (1986). Followup study on amyotrophic lateral sclerosis in Rochester, Minn., 1925 through 1984. Neuroepidemiology 5, 61-70.

Yuan, J., and Yankner, B.A. (2000). Apoptosis in the nervous system. Nature 407, 802-809.

Zhao, L., and Ackerman, S.L. (2006). Endoplasmic reticulum stress in health and disease. Curr Opin Cell Biol 18, 444-452.

Zhao, Z., Lange, D.J., Voustianiouk, A., MacGrogan, D., Ho, L., Suh, J., Humala, N., Thiyagarajan, M., Wang, J., and Pasinetti, G.M. (2006). A ketogenic diet as a potential novel therapeutic intervention in amyotrophic lateral sclerosis. BMC Neurosci 7, 29.

Zhong, X., and Pittman, R.N. (2006). Ataxin-3 binds VCP/p97 and regulates retrotranslocation of ERAD substrates. Hum Mol Genet 15, 2409-2420.

Zhu, S., Stavrovskaya, I.G., Drozda, M., Kim, B.Y., Ona, V., Li, M., Sarang, S., Liu, A.S., Hartley, D.M., Wu, D.C., et al. (2002). Minocycline inhibits cytochrome c release and delays progression of amyotrophic lateral sclerosis in mice. Nature 417, 74-78.

Zoghbi, H.Y., and Orr, H.T. (2000). Glutamine repeats and neurodegeneration. Annu Rev Neurosci 23, 217-247. 


\section{Curriculum vitae}

\section{David Herholz}

European Neuroscience Institute

Grisebachstrasse 5

37077 Göttingen, Germany

++4917662542020

e-mail: $\underline{\text { dherhol@gwdg.de }}$

Date of Birth:

07. 07. 1980.

Place of Birth:

Datteln, Germany

Nationality:

German

\section{EDUCATION}
$1987-1991$
Primary school, Lohschule Datteln
$1991-2000$
High school, Comenius Gymnasium Datteln
$2000-2001$
Civil service, St. Vinzens Krankenhaus Datteln
$2001-2007$
Diploma at the Faculty of Biology, Georg-August- University Göttingen
2007 - current
European Neuroscience Institute, Göttingen Laboratory of Developmental Neurobiology PhD thesis: "Profiling the inherent vulnerability of motor neuron subtypes"

\section{CONFERENCES AND PRESENTATIONS}

03/2008

05/2009

05/2009
ENI NET PhD symposium, Berlin, Germany Oral presentation: "Functional genetic investigation of distinct motor neuron types."

ENI-NET meeting, Heraklion, Greece

Poster presentation: "ALS-linked TDP-43 and neurotoxicity: Mutations, Truncations or inherent protein functions?"

Neurizons 2009, Goettingen, Germany Poster presentation: "Profiling the inherent vulnerability of motor neuron subtypes." 


\section{List of publications}

Voigt A. ${ }^{*}$ Herholz D. ${ }^{*}$, Fiesel FC., Kaur K., Müller D., Karsten P., Weber SS., Kahle PJ., Marquardt T. \& Schulz JB. (2010) TDP-43-mediated neuron loss in vivo requires RNA-binding activity. PLoS One. Aug 18;5(8):e12247.

${ }^{*}$ Those authors have contributed equally to this work 